\title{
BATF and IRF4 cooperate to counter exhaustion in tumor-infiltrating CAR T cells
}

\author{
Hyungseok Seo ${ }^{1,9,11}$, Edahí González-Avalos ${ }^{1,2,11}$, Wade Zhang1,3, Payal Ramchandani ${ }^{1,4,9}$, Chao Yang ${ }^{1}$, \\ Chan-Wang J. Lio ${ }^{1,10}$, Anjana Rao ${ }^{1,5,6,7,8 凶}$ and Patrick G. Hogan $\mathbb{1}^{1,7,8} \bowtie$
}

\begin{abstract}
The transcription factors nuclear factor of activated T cells (NFAT) and activator protein 1 (AP-1; Fos-Jun) cooperate to promote the effector functions of T cells, but NFAT in the absence of AP-1 imposes a negative feedback program of T cell hyporesponsiveness (exhaustion). Here, we show that basic leucine zipper ATF-like transcription factor (BATF) and interferon regulatory factor 4 (IRF4) cooperate to counter T cell exhaustion in mouse tumor models. Overexpression of BATF in CD8 ${ }^{+} T$ cells expressing a chimeric antigen receptor (CAR) promoted the survival and expansion of tumor-infiltrating CAR T cells, increased the production of effector cytokines, decreased the expression of inhibitory receptors and the exhaustion-associated transcription factor TOX and supported the generation of long-lived memory T cells that controlled tumor recurrence. These responses were dependent on BATF-IRF interaction, since cells expressing a BATF variant unable to interact with IRF4 did not survive in tumors and did not effectively delay tumor growth. BATF may improve the antitumor responses of CAR T cells by skewing their phenotypes and transcriptional profiles away from exhaustion and towards increased effector function.
\end{abstract}

C $\mathrm{D}^{+} \mathrm{T}$ cells that encounter antigen together with effective costimulatory signals mount strong effector responses that are able to clear pathogen-infected cells and tumor cells. In contrast, $\mathrm{CD}^{+} \mathrm{T}$ cells that infiltrate solid tumors and are exposed to prolonged antigen stimulation in the absence of adequate costimulation enter a hyporesponsive (exhausted or dysfunctional) state in which they do not effectively destroy tumor cells ${ }^{1-3}$. Exhausted $\mathrm{T}$ cells express high levels of inhibitory receptors, including PD-1, T cell immunoglobulin and mucin domain-containing protein 3 (TIM3), and lymphocyte activation gene 3 protein (LAG3); low levels of effector proteins, including cytokines and granzymes; and transcription factors of the nuclear receptor subfamily 4 group A (NR4A) and thymocyte selectionassociated high-mobility group box (TOX) families, that act to impose exhaustion ${ }^{4-10}$.

The effector and exhaustion responses of $\mathrm{CD}^{+} \mathrm{T}$ cells are both initiated by $\mathrm{T}$ cell receptor (TCR) signaling, and the transcription factor nuclear factor of activated T cells (NFAT) plays a pivotal role in both responses, with the balance between them depending on the transcriptional partners of NFAT. During acute immune responses, NFAT predominantly induces the effector program by cooperating with its partner transcription factor activator protein $1(\mathrm{AP}-1)^{11,12}$. The effector program also requires independent binding of AP-1 and nuclear factor- $\mathrm{KB}$ transcription factors to other sites $^{13}$. Classical AP-1 is comprised of heterodimers of the basic region-leucine zipper (bZIP) transcription factors FOS and $\mathrm{JUN}^{14}$, but heterodimers of other FOS/JUN family proteins can also cooperate with NFAT ${ }^{13}$. In contrast, whereas NFAT remains nuclear for many hours in antigen-stimulated T cells ${ }^{15}$, the expression and activity of FOS and JUN family members is not sustained ${ }^{14}$. Under these conditions,
NFAT acts predominantly in the negative feedback program to induce genes associated with exhaustion and dysfunction ${ }^{4-10}$.

Recent attention has focused on two downstream targets of NFAT-the NR4A family of orphan nuclear receptors and the TOX family of high-mobility group box DNA-binding proteins ${ }^{16}$. Depletion of NR4A1 (ref. ${ }^{4}$ ), all three NR4A family members ${ }^{5}$, TOX $^{10}$ or TOX and TOX2 proteins ${ }^{6}$ confers robust antitumor responses on $\mathrm{CD}^{+}$tumor-infiltrating $\mathrm{T}$ lymphocytes (TILs). Mechanistically, TOX/TOX2 or NR4A depletion prevents some of the chromatin and transcriptional changes characteristic of exhaustion, and partially rescues the effector activity of TILs ${ }^{4-10}$. The onset of exhaustion coincides with decreased chromatin accessibility of regions enriched for AP-1/bZIP motifs and a decrease in the expression of many bZIP proteins ${ }^{5,6}$. Based on these findings, we hypothesized that restoring AP-1 expression and function in $\mathrm{CD}^{+}$TILs would allow the formation of activating NFAT-AP-1 complexes and prevent $\mathrm{CD}^{+} \mathrm{T}$ cell exhaustion ${ }^{5,6,12,17}$. Recently, $\mathrm{CD}^{+} \mathrm{T}$ cells expressing a chimeric antigen receptor (CAR) for a tumor antigen and overexpressing JUN were shown to be more effective than control CAR $\mathrm{T}$ cells at slowing tumor growth ${ }^{18}$.

The transcription factor basic leucine zipper ATF-like transcription factor (BATF) and its partners interferon regulatory factor 4 (IRF4) and IRF8 are also induced by TCR signaling ${ }^{19-24}$. Like NFAT, BATF can contribute both to effector function and to exhaustion, depending on the biological context ${ }^{12,19,25,26}$. Here, we show that overexpressed BATF can cooperate with IRF4 to counteract the development of $\mathrm{T}$ cell exhaustion. Overexpression of BATF in $\mathrm{CD} 8^{+}$ CAR T cells led to a marked increase in the survival and expansion of TILs; increased the ability of the CAR TILs to produce cytokines and granzymes after stimulation; and reduced their expression of

'Division of Signaling and Gene Expression, La Jolla Institute for Immunology, La Jolla, CA, USA. 'Bioinformatics and Systems Biology Graduate Program, University of California, San Diego, La Jolla, CA, USA. ${ }^{3}$ Bioengineering Graduate Program, Bioengineering Department, University of California, San Diego, La Jolla, CA, USA. ${ }^{4}$ Contiguous BS/MS Program, Biology Department, University of California, San Diego, La Jolla, CA, USA. ${ }^{5}$ Department of Pharmacology, University of California, San Diego, La Jolla, CA, USA. ${ }^{6}$ Sanford Consortium for Regenerative Medicine, La Jolla, CA, USA. ${ }^{7}$ Moores Cancer Center,

University of California, San Diego, La Jolla, CA, USA. ${ }^{8}$ Center for Cancer Immunotherapy, La Jolla Institute for Immunology, La Jolla, CA, USA. ${ }^{9}$ Present address: Novartis Institutes for BioMedical Research, Cambridge, MA, USA. ${ }^{10}$ Present address: Department of Microbial Infection and Immunity, College of Medicine, The Ohio State University, Columbus, OH, USA. "These authors contributed equally: Hyungseok Seo, Edahí González-Avalos.

凶e-mail:arao@lji.org; phogan@lji.org 
inhibitory cell-surface receptors and the exhaustion-associated transcription factor TOX. Tumor-bearing mice that had previously received BATF-transduced $\mathrm{CD}^{+}{ }^{+} \mathrm{T}$ cells and rejected the tumor developed long-lived memory $\mathrm{T}$ cells that controlled tumor recurrence. There is substantial interest in manipulating CAR T cells to control tumors more effectively, and BATF overexpression potentially represents a simple and therapeutically effective method for achieving this desired outcome.

\section{Results}

BATF-transduced CAR T cells exhibit enhanced tumor rejection. A preliminary screen for transcription factors that could enhance NFAT-AP-1 activity in CD8 ${ }^{+} \mathrm{T}$ cells led us to JUN, MAFF and BATF (Extended Data Fig. 1) and raised the question of whether JUN, MAFF or BATF could confer a functional antitumor advantage on $\mathrm{CD}^{+} \mathrm{CAR} \mathrm{T}$ cells in vivo.

$\mathrm{CD}^{+} \mathrm{T}$ cells were retrovirally transduced with a CAR directed against human CD19 (hCD19) ${ }^{5,6}$, together with a retroviral expression vector for JUN, MAFF or BATF or an empty (pMIG) retrovirus control, and adoptively transferred $7 \mathrm{~d}$ after tumor inoculation into C57BL/6J mice bearing the B16F0-hCD19 tumor. Transduction yielded very high expression of each transcription factor compared with endogenous protein, but did not alter the expression of the Myc-tagged CAR (Extended Data Fig. 2). Mice adoptively transferred with control pMIG- or MAFF-transduced CAR T cells showed tumor sizes similar to those of mice treated with phosphate-buffered saline (PBS) alone, whereas mice receiving JUN-transduced CAR $\mathrm{T}$ cells showed a variable delay in tumor growth (Fig. 1a,b). Mice injected with BATF-transduced CAR T cells showed a notable delay in tumor growth, as well as a significant improvement in long-term survival compared with all other groups (Fig. 1a-c). The findings with BATF-transduced CAR T cells were confirmed in replicate B16 melanoma experiments and in experiments with an MC38-hCD19 colon adenocarcinoma (Extended Data Fig. 2).

To further explore the antitumor responses of BATF-transduced CAR T cells, we transferred pMIG- or BATF-transduced CAR T cells into tumor-bearing recipient mice $12 \mathrm{~d}$ after tumor inoculation (at which time the tumor was large and well established) and harvested TILs $8 \mathrm{~d}$ after CAR T cell transfer. Mice given BATF-transduced CAR T cells showed substantially slower tumor growth compared with mice given control pMIG-transduced CAR T cells (Fig. 1d). BATF-transduced CAR TILs, identified by expression of the Thy1.1 reporter, showed a striking increase in frequency in the tumor compared with control pMIG-transduced cells (Fig. 1e).

BATF overexpression directs CAR TILs away from exhaustion. Consistent with their expansion and function in the tumor microenvironment, BATF-transduced CAR TILs showed decreased immunochemical staining of all of the inhibitory receptors tested; a marked increase in the proliferation marker Ki67; decreased expression of the naive/memory markers CD127 and CD62L; increased expression of CD44; expression of KLRG1 in a subpopulation of cells; and decreased expression of TOX, a transcription factor strongly associated with CD8 ${ }^{+} \mathrm{T}$ cell exhaustion ${ }^{6-10}$ (Fig. 1f-i and Extended Data Fig. 2). Induction of interferon- $\gamma$ and expression of granzyme B and CD107a were significantly increased after phorbol 12-myristate 13-acetate/ionomycin stimulation in BATF-transduced compared with control pMIG CAR TILs (Extended Data Fig. 2).

Mass cytometry confirmed these findings and provided evidence that additional markers of previously activated or effector $\mathrm{CD}^{+}$ $\mathrm{T}$ cells were upregulated (Fig. 2). TOX and PD-1 were coexpressed in control pMIG-transduced CAR TILs, as in other exhausted $\mathrm{CD}^{+} \mathrm{T}$ cells ${ }^{6-10}$, but the PD-1 ${ }^{\text {high }}$ TOX ${ }^{\text {high }}$ population was absent in BATF-transduced CAR TILs (Fig. 2c). Conversely, inducible T cell costimulator (ICOS) and granzyme B expression were strongly correlated in BATF-transduced but not pMIG-transduced CAR TILs (Fig. 2d), suggesting the presence of an effector-like TIL subset elicited in part by BATF overexpression.

A progenitor-like $\mathrm{T}$ cell population expressing the transcription factor T cell-specific factor 1 (TCF1) sustains the immune response against both tumors and chronic viral infections and underlies the proliferative response to checkpoint blockade immunotherapy ${ }^{27-31}$. Among both pMIG- and BATF-transduced CAR TILs, TCF $1^{+}$cells remained TIM $3^{\text {low }}$ and granzyme $B^{\text {low }}$ (Fig. 2e,f), consistent with a progenitor-like role. The $\mathrm{TCF} 1^{+}$subset constituted a reduced percentage of BATF-overexpressing TILs, but still an ample number of cells to account for their survival and effector function in the tumor (Fig. 2e-h).

BATF-transduced CAR T cells persist after tumor regression. We asked whether CAR TILs persisted in mice that had rejected an initial tumor, and if so whether they conferred protection against rechallenge with the same tumor. B16F0-hCD19 tumor cells were injected on the opposite flank of the five surviving mice from the previous experiment, with a corresponding tumor-naive cohort of five age-matched C57BL/6 mice as controls. Tumors grew in the tumor-naive group as expected, but did not develop (four mice) or quickly regressed (one mouse) in the previously challenged group (Fig. 3a). Thy $1.1^{+} \mathrm{CD}^{+} \mathrm{CAR} \mathrm{T}$ cells were recovered from the draining lymph nodes and spleen of all five surviving mice of the BATF-transduced CAR group (Fig. 3b). The recovered CAR T cells displayed characteristics similar to central memory $\mathrm{CD}^{+} \mathrm{T}$ cells, including expression of CD27, CD44, TCF1, CD62L and CD127 (Fig. 3c,d and Extended Data Fig. 3). Tumor rechallenge gave a similar result in the replicate B16-hCD19 experiment (Extended Data Fig. 3). Thus, BATF-transduced CAR T cells persisted for many weeks after tumor clearance and acquired features of memory T cells.

Fig. 1 | Antitumor effects of CAR T cells ectopically expressing bZIP transcription factors. a-c, $1 \times 10^{5}$ B16F0-hCD19 tumor cells were injected subcutaneously into the left flank of C57BL/6 mice at day 0 in $100 \mu \mathrm{IBS}(n=12)$. Then, $3 \times 10^{6}$ control pMIG- $(n=16)$, JUN- $(n=14)$, MAFF- $(n=7)$ or BATF-transduced $(n=24)$ CAR T cells were adoptively transferred by retro-orbital injection at day 7. $\mathbf{a}, \mathbf{b}$, Tumor growth rates (a) and tumor sizes (b) at day 20 (dashed vertical line in a) for individual mice. c, Mouse survival curves up to $100 \mathrm{~d}$ after tumor inoculations. $\mathbf{d}$-i, $1 \times 10^{5}$ B16F0-hCD19 tumor cells were subcutaneously injected into the left flank of C57BL/ 6 mice at day 0 . Then, $1.5 \times 10^{6}$ pMIG- $(n=5)$ or BATF-transduced $(n=5)$ CAR T cells were adoptively transferred at day 12 . TILs were isolated at day 20. d, Tumor growth curves for individual mice (dashed lines) and the average of all tumor growth curves per group (bold lines). e, Top: contour plot of flow cytometry data for the CAR TILs. Bottom: percentage of CAR TILs relative to total CD8 ${ }^{+}$ TILs in the tumor (left) and normalized number of CAR TILs per tumor, obtained by dividing the absolute number of CAR TILs by the tumor area (right). f, Median fluorescence intensity (MFI) of the entire flow plot for the indicated inhibitory receptors from each group of CAR TILs. $\mathbf{g}$, Top: representative contour plots of PD-1 and Tim3 expression on CAR TILs. Bottom: percentage of cells in each of the indicated quadrants (Q1=PD- high $^{\text {TIM3 }}{ }^{\text {low; }}$ Q Q2 = PD-

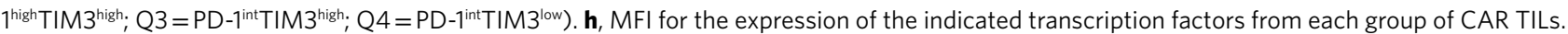
$\mathbf{i}$, MFI fold change between PMIG- and BATF-transduced CAR TILs. Each circle in $\mathbf{b}$ and $\mathbf{e}$-i represents one mouse, horizontal bars in $\mathbf{b}$ and $\mathbf{i}$ indicate mean values, and the bar graphs in $\mathbf{e}-\mathbf{h}$ represent means \pm s.e.m. The data in $\mathbf{a}-\mathbf{c}$ and $\mathbf{d}$-i were obtained from three and two independent experiments, respectively. Statistical significance was determined by one-way analysis of variance (ANOVA) (b), log-rank Mantel-Cox test (c), two-way ANOVA (d) or two-tailed unpaired Student's $t$-test $(\mathbf{e}-\mathbf{h}) .{ }^{\star} P \leq 0.05 ;{ }^{\star \star} P \leq 0.01 ;{ }^{\star \star \star} P \leq 0.001 ;{ }^{\star \star \star \star} P \leq 0.0001$. 
BATF directs human CAR $T$ cells towards an effector-like phenotype. An important question is whether BATF overexpression exerts similar effects in human $\mathrm{T}$ cells. We transduced human $\mathrm{CD}^{+} \mathrm{T}$ cells with a human CAR construct targeting hCD19 (ref. ${ }^{32}$ ) and with a vector encoding human BATF or its empty-vector control (Extended Data Fig. 4). The levels of
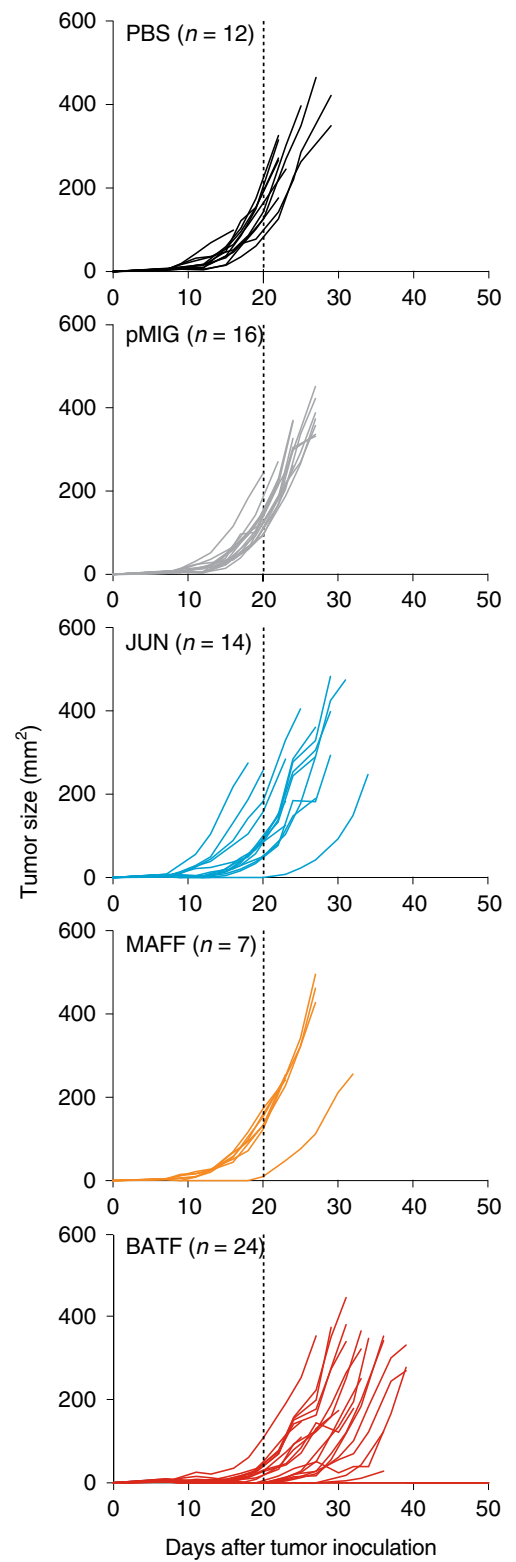

b

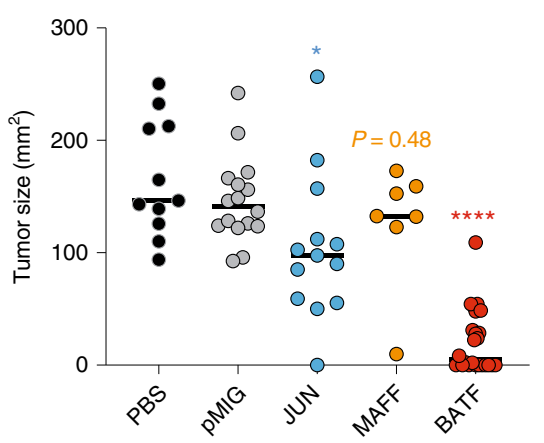

d

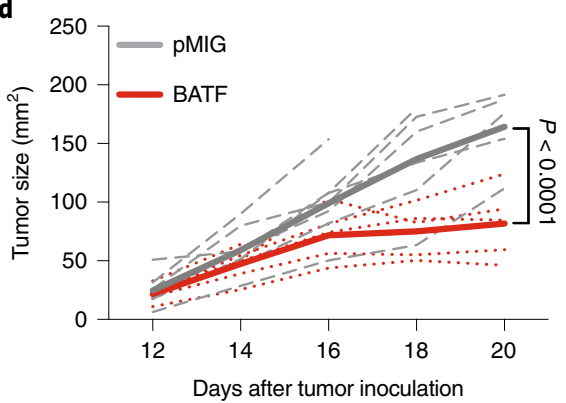

f

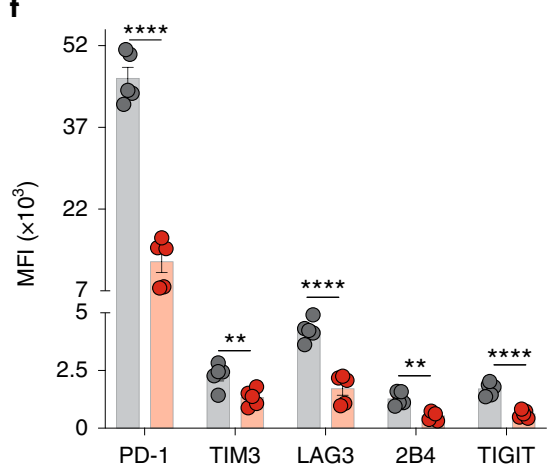

c

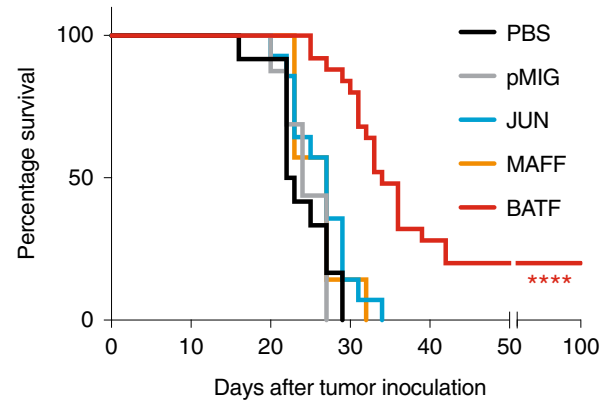

e
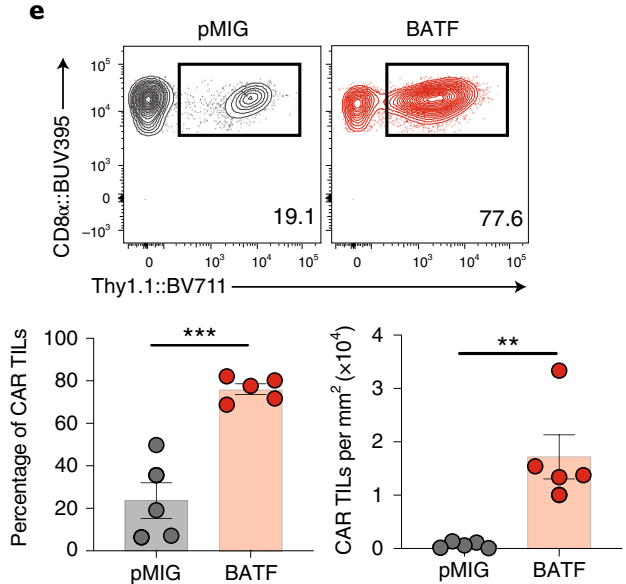

$\mathbf{g}$
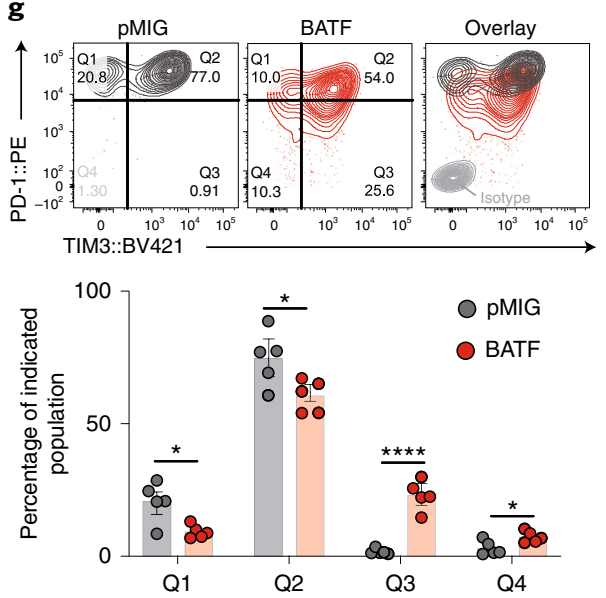

h

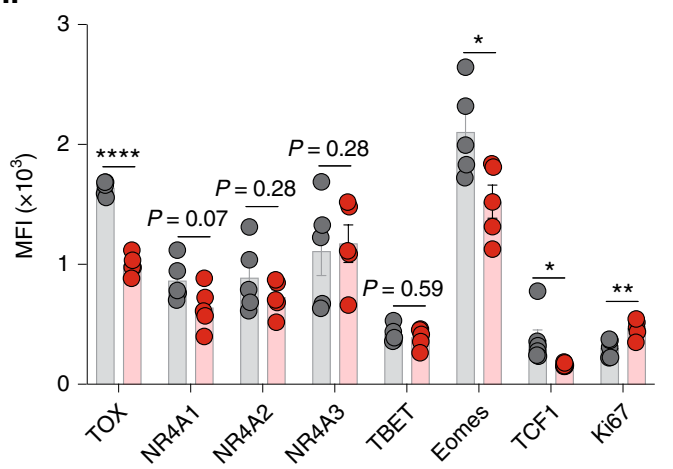

i

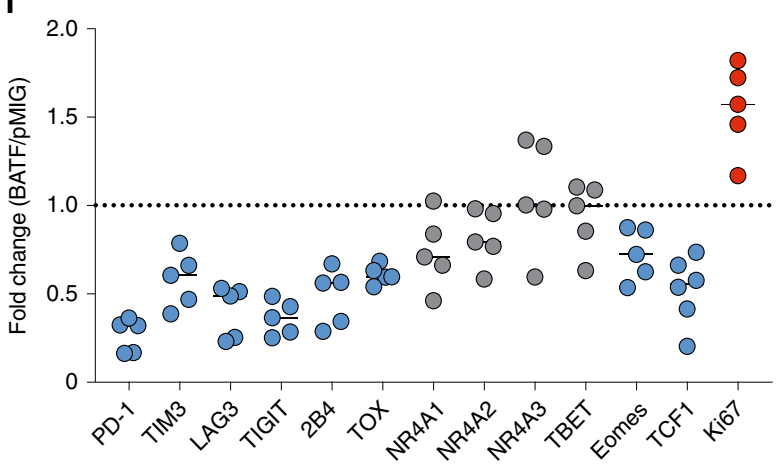


a
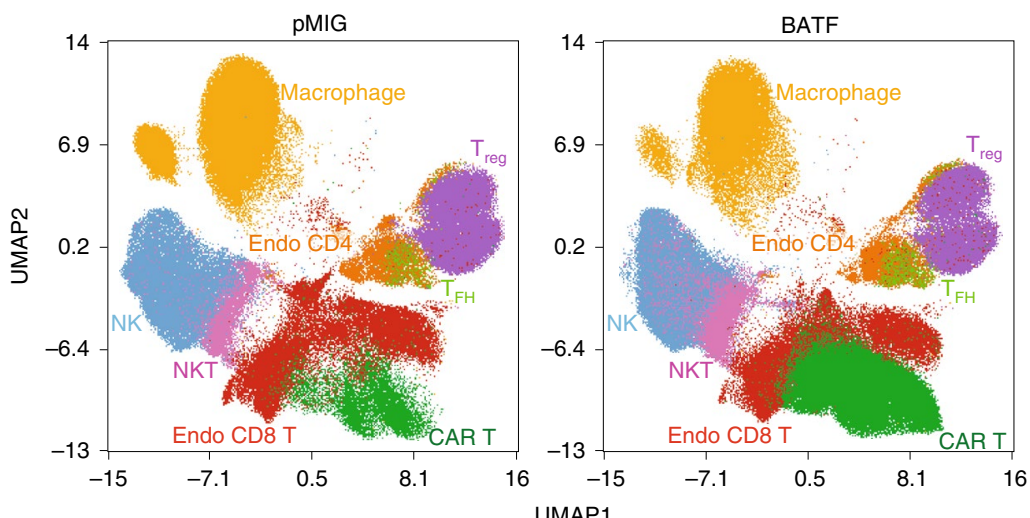

b

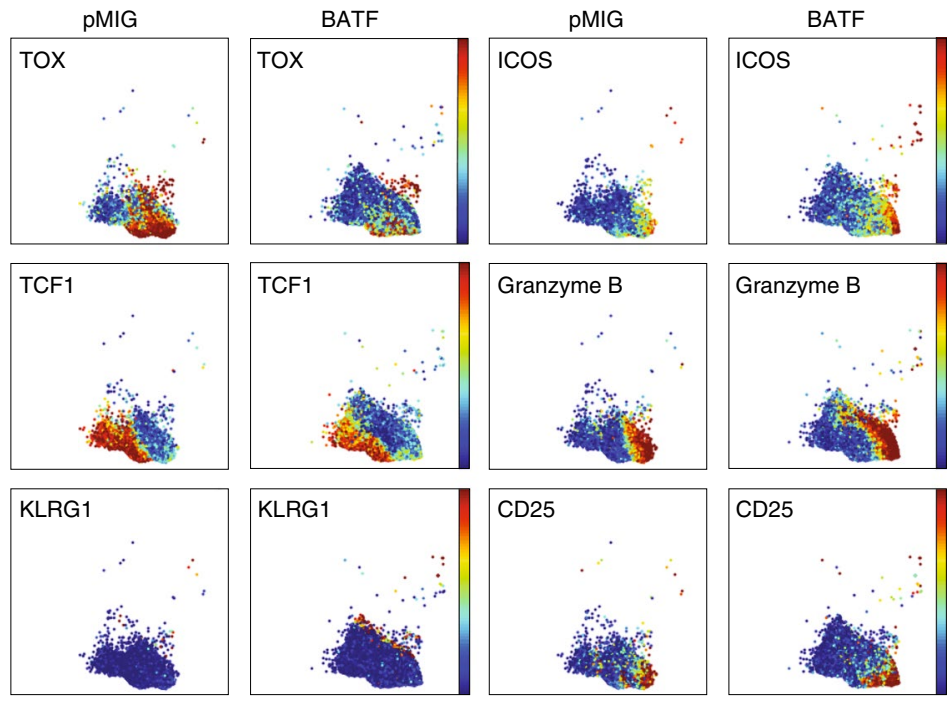

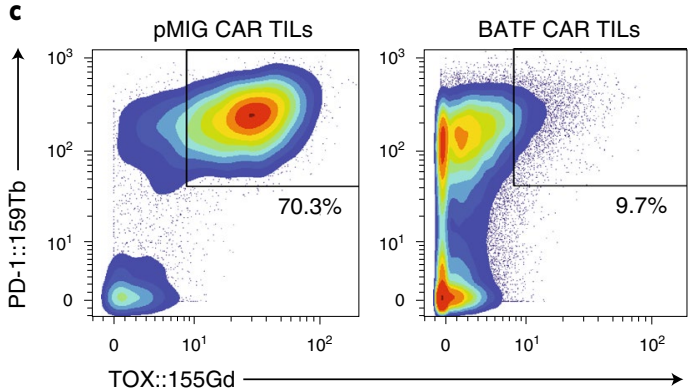

d

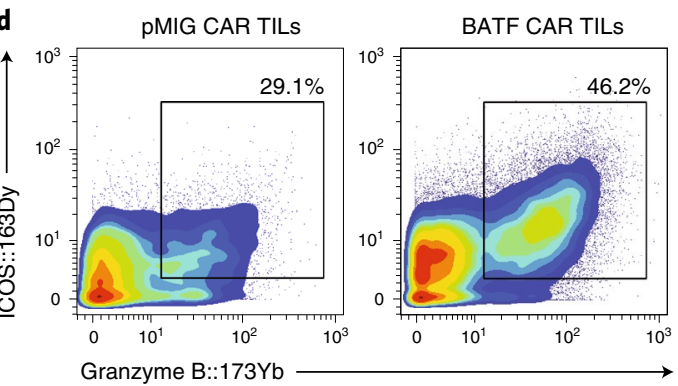

e
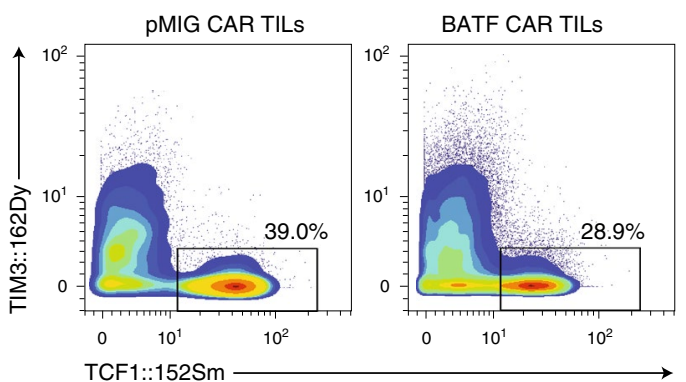

f

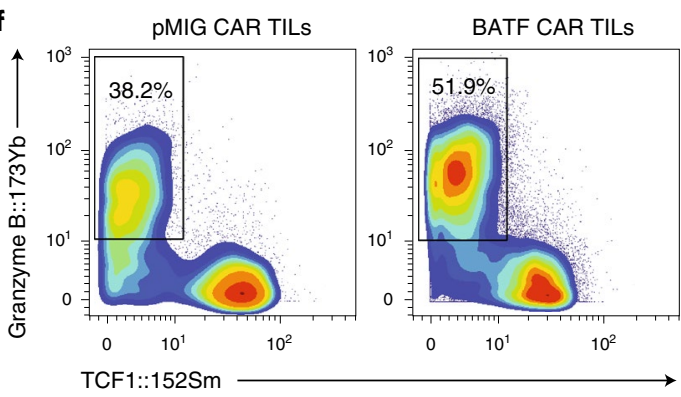

g

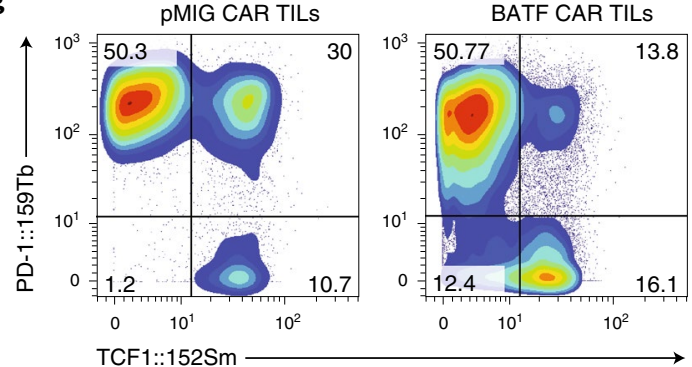

h

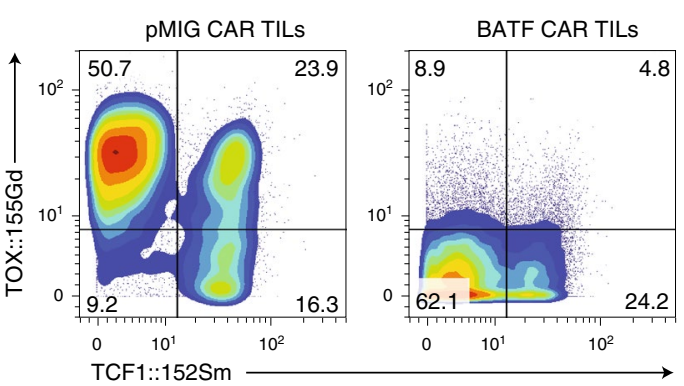

Fig. 2 | High-dimensional single-cell characterization of pMIG- and BATF-transduced CAR TILs by mass cytometry (CyTOF). a-h, $1 \times 10^{5}$ B16F0-hCD19 tumor cells were injected subcutaneously into the left flank of C57BL/ 6 mice at day 0 . Then, $1.5 \times 10^{6} \mathrm{pMIG}$ - or BATF-transduced CAR T cells were adoptively transferred at day 12. TILs were isolated at day 20 and stained with metal-conjugated antibodies for mass cytometry, performed at day 21 using a CyTOF mass spectrometer. The gating strategy is detailed in Extended Data Fig. 3a. a, Plots showing UMAP views providing comprehensive single-cell analysis and distinguishing TIL subpopulations. Endo, endogenous; NK, natural killer cells; NKT, natural killer T cells; $T_{\mathrm{FH}}$, follicular helper T cells; $\mathrm{T}_{\text {reg, }}$ regulatory T cells. $\mathbf{b}$, Detection of the indicated markers on individual pMIG or BATF CAR TILs, visualized in the UMAP representation using a color scale from violet (lowest CyTOF signal) to red (highest CyTOF signal). c-h, Contour plots analyzing pairwise expression of the indicated markers (c, PD-1 and TOX; d, ICOS and Granzyme B; e, TIM3 and TCF1; f, Granzyme B and TCF1; $\mathbf{g}$, PD-1 and TCF1; $\mathbf{h}$, TOX and TCF1) on pMIG or BATF CAR TILs. The data are representative of two biological experiments. Each group of samples was pooled from ten mice. 


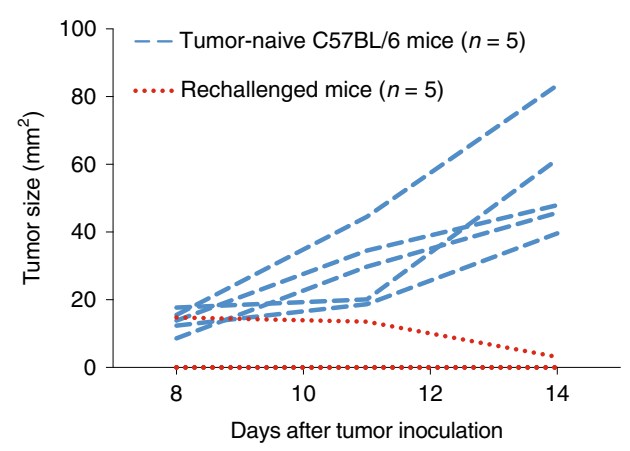

C
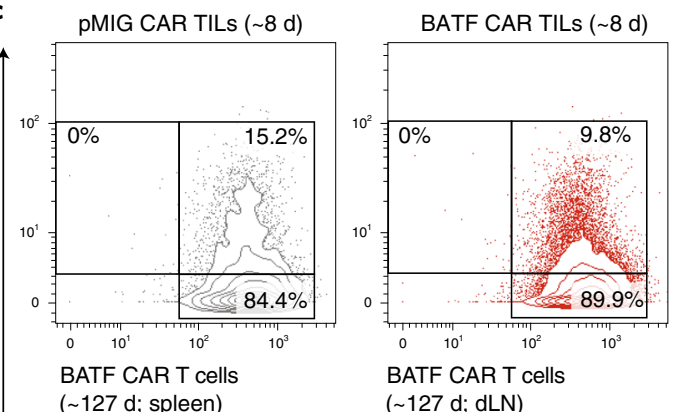

( 127 d; spleen)
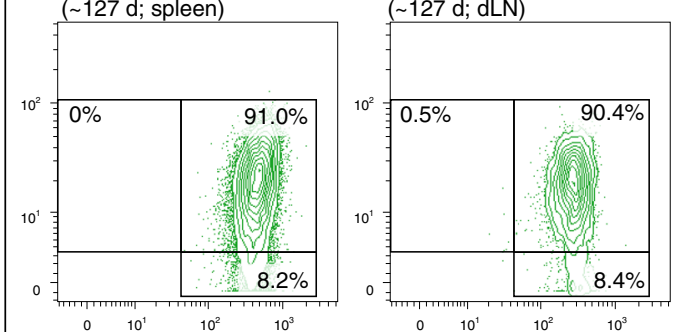

Naive B6 spleen (CD8 T)
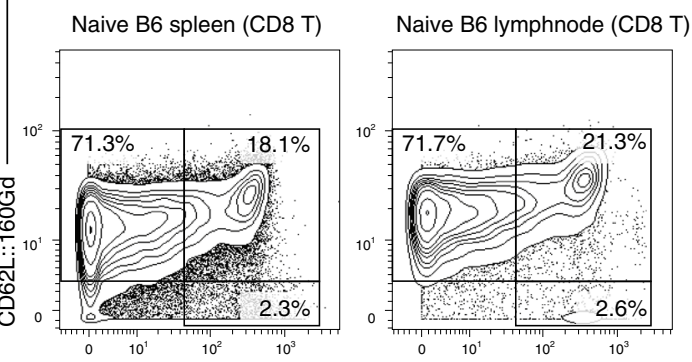

b

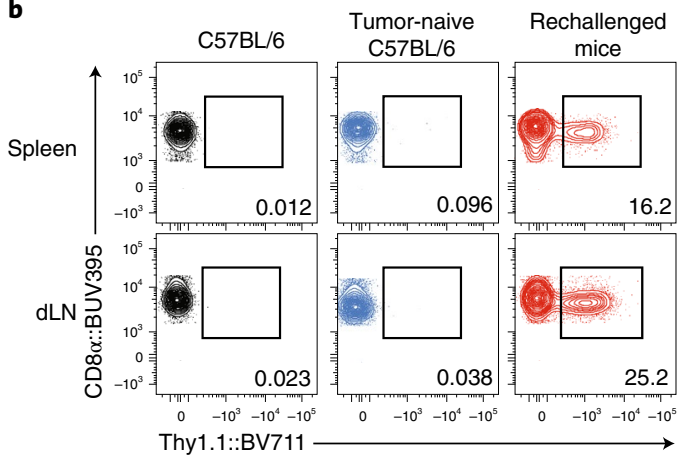

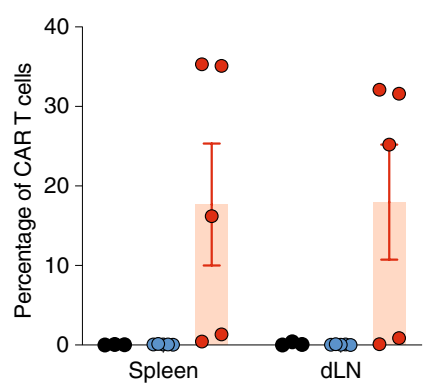

d
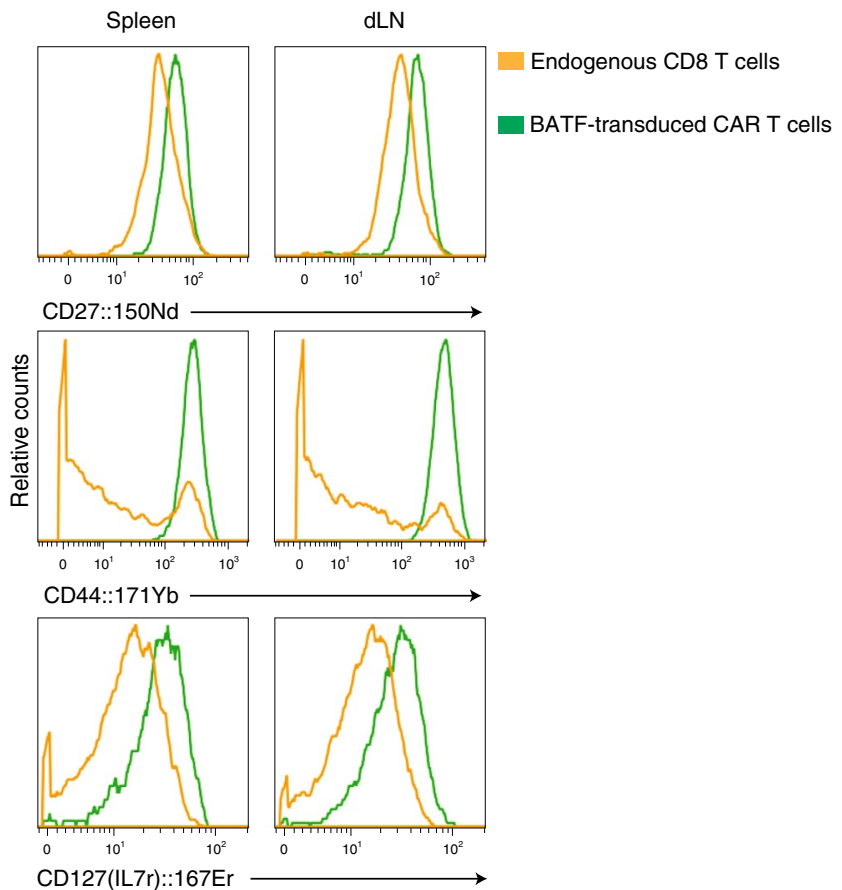

CD44::171Yb

Fig. 3 | BATF-transduced CAR T cells confer a memory response against tumor rechallenge and exhibit a memory phenotype. a-d, $1 \times 10^{5}$ B16F0-hCD19 tumor cells were injected subcutaneously into the right flank of C57BL/6 mice $(n=5)$ to yield the tumor-naive control group, or into tumor-free mice from the experiment in Fig. 1c $(n=5)$ that had rejected an initial B16F0-hCD19 tumor and had survived until day 120 after the first tumor injection (rechallenged group). Spleens and draining lymph nodes were harvested $14 \mathrm{~d}$ after tumor inoculation or tumor rechallenge. $\mathbf{a}$, Tumor growth curves for individual mice (tumor-naive C57BL/6 mice are represented by blue dashed lines; rechallenged mice are represented by red dotted lines). No tumor growth was detected in four of the rechallenged mice. $\mathbf{b}$, Left: representative contour plots showing the frequencies of CAR T cells in splenocytes and draining lymph node (dLN) cells from a fresh control C57BL/6 mouse that did not receive tumor cells, a tumor-bearing C57BL/6 mouse (tumor-naive control group) and a rechallenged mouse. Right: percentage of CAR T cells relative to total CD8 $8^{+} \mathrm{T}$ cells harvested from spleen and lymph nodes for the five mice in each group. The mouse with the lowest frequency of CAR T cells was the one in which the rechallenge tumor had been present initially but then regressed. Each circle represents one mouse, and the bar graph represents means \pm s.e.m. The data are representative of two biological experiments (see Extended Data Fig. 3e). c, Contour plots for CD62L (y axis) and CD44 (x axis) expression. Top: CD8 ${ }^{+}$T cells from BATF- and pMIG-transduced CAR TILs $8 \mathrm{~d}$ after transfer of CAR T cells from the CyTOF experiment of Fig. 2 and Extended Data Fig. 3. Middle: BATF-transduced CAR T cells from the spleens and draining lymph nodes of rechallenged mice $127 \mathrm{~d}$ after CAR T cell adoptive transfer. Bottom: splenocytes and lymphocytes from the draining lymph nodes of fresh control C57BL/6 mice. $\mathbf{d}$, Histograms plotting the CyTOF signals of the indicated markers in endogenous CD8 ${ }^{+} \mathrm{T}$ cells and BATF-transduced CAR T cells from rechallenged mice. Samples for each group analyzed in $\mathbf{c}$ and $\mathbf{d}$ were pooled from five mice.

CAR expression were comparable in the experimental and control cells. When cultured together with hCD19-bearing tumor cells to assess effector function, BATF-overexpressing human CAR T cells proliferated more than control CAR T cells and showed enhanced cytokine expression, granzyme B expression and cytotoxicity. 
BATF-IRF4 interaction is essential for antitumor responses. A prominent effect of BATF in $\mathrm{CD}^{+} \mathrm{T}$ cells is to recruit IRF to AP-1-IRF composite element (AICE) sites in DNA, where a heterodimer of BATF and a JUN family transcription factor binds cooperatively with IRF4 or IRF8 (refs. ${ }^{20-25,33}$ ). We introduced the His55Gln/Lys63Asp/Glu77Lys (HKE) substitutions, which suppress the interaction between BATF and IRF4 (refs. ${ }^{20,24,33}$ ), into the BATF sequence encoded by the expression plasmid. BATF-HKE was expressed in $\mathrm{CD}^{+} \mathrm{T}$ cells at levels similar to wild-type BATF (Fig. 4a) and retained DNA binding, as reported previously ${ }^{20,33}$ and confirmed in the chromatin immunoprecipitation sequencing (ChIP-seq) analyses described below. Tumors developing in mice given HKE variant-transduced CAR T cells at day 7 after tumor inoculation were significantly larger than those in mice given wild-type BATF-transduced CAR T cells (Fig. 4b and Extended Data Fig. 5), and the survival of the mice was significantly lower (Fig. 4c). Thus, selectively impairing the interaction of BATF with IRF4 strongly attenuated the ability of BATF-overexpressing CAR $\mathrm{T}$ cells to control the tumor.

HKE variant-transduced CAR $\mathrm{T}$ cells adoptively transferred at day 12 after tumor inoculation were likewise ineffective at controlling tumor growth, and this ineffectiveness was associated with a marked decrease in the frequency and number of CAR TILs (Fig. 4d-g). To follow TIL expansion in vivo, we transferred CAR $\mathrm{T}$ cells into tumor-bearing mice at day 12 after tumor inoculation and monitored TIL numbers and phenotypes on days 13, 16, 19 and 22 (Fig. 4h-k and Extended Data Fig. 5). The striking expansion of BATF-transduced CAR TILs compared with control TILs and the contrasting low numbers of HKE variant-transduced TILs were already obvious $4 \mathrm{~d}$ after transfer and became even more pronounced at later times. The fraction of $\mathrm{PD}-1^{+} \mathrm{TIM}^{+}$cells among the few CAR TILs expressing the HKE variant was low compared with controls on day 16 , and progressively declined from days 16-22 in parallel with an increase in PD-1- ${ }^{-} \mathrm{TIM}^{-}$cells.

CAR T cells carrying a germline deletion of BATF (Bat $f$ knockout) likewise had no effect on tumor growth and exhibited a striking paucity of TILs (Extended Data Fig. 5). PD-1 expression in the few recovered cells was substantially diminished compared with wild-type CAR TILs, and PD-1+TIM3 ${ }^{+}$CAR TILs were almost absent. Moreover, Batf knockout CAR TILs displayed a decreased frequency of PD- $1^{+}$TOX ${ }^{\text {high }}$ cells and a prominent group of naive-like TCF $1^{+}$TIM $3^{-}$cells. Collectively, these data reveal that BATF and the BATF-IRF4 interaction are absolutely required for the survival and expansion of BATF-transduced CAR T cells in tumors, and that endogenous BATF3 does not compensate for the germline loss of BATF.

Coexpression of IRF4 with BATF hampers the antitumor response. Given these data, we asked whether coexpressing IRF4 with BATF would further improve the antitumor responses of CD8 ${ }^{+}$ TILs. OT-I cells expressing BATF alone, IRF4 alone or BATF + IRF4 were injected on either day 7 or day 12 after tumor inoculation, and tumor size was measured subsequently (Extended Data Fig. 6). All three types of transduced cells initially slowed tumor growth. Overexpression of either BATF alone or BATF with IRF4 resulted in striking TIL expansion, decreased expression of the exhaustion markers PD-1, TIM3 and TOX and increased expression of granzymes and effector cytokines. However, the effects of overexpressing BATF or IRF4 individually were distinct. Overexpressing BATF alone was more effective than overexpressing IRF4 alone at promoting TIL expansion and downregulating TOX, PD-1 and TIM3, whereas overexpressing IRF4 alone led to more cytokine and granzyme B expression upon stimulation.

Because BATF binds DNA as a heterodimer with JUN family members, we compared antitumor responses in OT-I cells transduced with BATF alone, JUN alone or BATF + JUN (Extended Data Fig. 6). JUN overexpression in OT-I cells did not substantially slow the growth of B16F10-OVA tumors beyond that observed with control pMIG-transduced OT-I cells. In contrast, mice given OT-I cells transduced with BATF alone showed a strong reproducible delay in tumor growth, and mice given OT-I cells transduced with both BATF and Jun showed, surprisingly, a less impressive delay.

Distinctive transcriptional profile of BATF-transduced CAR TILs. Transcriptional profiling (Supplementary Table 1) highlighted Ifnarl and the genes encoding its downstream signaling effectors Stat 1 and Stat 3 , as well as Il12rb2, as genes differentially upregulated in BATF-overexpressing TILs compared with control TILs (Fig. 5a). These differences may render the BATF-overexpressing cells more receptive to interferon- $\alpha / \beta$ and interleukin-12 (IL-12) signals that promote effector and effector/memory function ${ }^{34,35}$, and may account for the enhanced effector function of BATF-overexpressing $\mathrm{T}$ cells in the tumor, as well as the ability to generate memory CAR $\mathrm{T}$ cells. Other genes related to effector function (Icos and $\mathrm{Gzma} / \mathrm{b} / \mathrm{c}$ ) showed increased messenger RNA (mRNA) expression (Fig. 5a), consistent with increased protein levels of ICOS and granzyme B after stimulation. We also observed increased expression of mRNAs encoding CCL5, CCR2, CXCR3 and CXCR6 (chemokines and chemokine receptors that are upregulated in activated/effector $\mathrm{CD}^{+}$ $\mathrm{T}$ cells and that promote the trafficking of CD8 $\mathrm{T}^{+}$cells to tumors and sites of inflammation; Fig. 5a) and decreased expression of mRNA encoding CCR7 (a chemokine receptor that is typically downregulated in effector $\mathrm{CD} 8^{+} \mathrm{T}$ cells). Perhaps most importantly, and again in line with the protein data, BATF-transduced CAR TILs displayed decreased expression of Tox mRNA, indicating a break in a crucial transcriptional link on the pathway to exhaustion. These observed changes are consistent with a transcriptional bias of the

Fig. 4 | The BATF-IRF interaction is required for CAR T cell survival, expansion and antitumor responses. a, Expression of endogenous BATF in pMIG-transduced cells and of BATF and BATF-HKE in retrovirally transduced CD8 ${ }^{+} T$ cells. The dashed line represents the isotype control. b,c, Experimental protocol as in Fig. 1a-c. b, Tumor sizes in individual mice at day 20. c, Survival curves. Data for PBS, pMIG and BATF are replotted from Fig. 1c, since the BATF-HKE variant $(n=12)$ was analyzed in the same experimental series. $\mathbf{d}-\mathbf{g}$, The experimental protocol was as in Fig. 1d-i, except with pMIG- $(n=7)$, BATF- $(n=6)$ or BATF-HKE-transduced $(n=6)$ CAR T cells. $\mathbf{d}$, Tumor growth curves for individual mice (dashed lines) and the averages for all mice in each group (bold lines). e, Representative contour plots of CD $8 \alpha$ and Thy 1.1 expression in the isolated TILs. The Thy 1.1 reporter marks CAR T cells. $\mathbf{f}$, Percentage of CAR TILs among CD8 ${ }^{+}$T cells. $\mathbf{g}$, Number of CAR TILs normalized to tumor size. h-k, $1 \times 10^{5}$ B16FO-hCD19 tumor cells were injected subcutaneously into the left flank of C57BL/6 mice at day 0 . Then, the indicated CAR T cells were adoptively transferred by retro-orbital injection on day 12. TILs were isolated on days 13, 16, 19 and 22. No CAR TILs were observed on day 13,1d after adoptive transfer. h,i, Percentages of CAR TILs (h) and normalized numbers of CAR TILs (i) on the indicated days. $\mathbf{j}$, Representative contour plots of PD-1 and TIM3 expression on the CAR TILs, as assessed by flow cytometry. $\mathbf{k}$, Frequencies of the indicated PD-1- and TIM3-expressing populations. Each circle in $\mathbf{b}$ and $\mathbf{f}-\mathbf{i}$ represents one mouse, horizontal bars in $\mathbf{b}$ indicate mean values, and the bar graphs in $\mathbf{f}-\mathbf{i}$ represent means \pm s.e.m. The data in $\mathbf{a}$ and $\mathbf{h}-\mathbf{k}$ are representative of two independent experiments. The data in $\mathbf{b}$ and $\mathbf{c}$ were obtained from three independent biological experiments. The data in $\mathbf{d}-\mathbf{g}$ were obtained from two independent biological experiments. Statistical significance was determined by one-way ANOVA (b,f and $\mathbf{g}$ ), log-rank Mantel-Cox test (c) or two-way ANOVA (d,h and $\mathbf{i})$. ${ }^{\star} P \leq 0.05 ;{ }^{* \star} P \leq 0.01 ;{ }^{* \star \star \star} P \leq 0.0001$ 
BATF-transduced CAR TILs towards increased tumor infiltration, increased expansion within tumors, increased effector function and decreased propensity to exhaustion.
Chromatin changes elicited by BATF overexpression. To distinguish early changes initiated by overexpressed BATF in transduced cells before transfer from subsequent changes induced in a

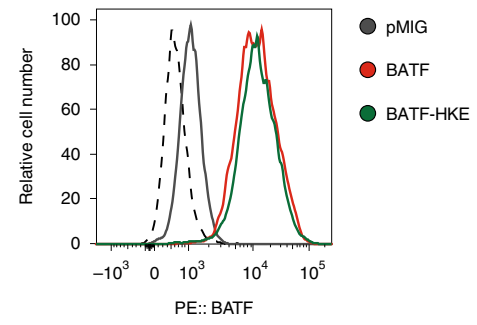

d

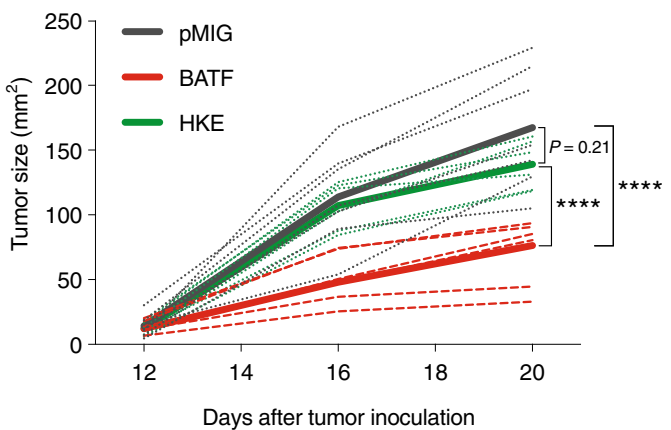

b

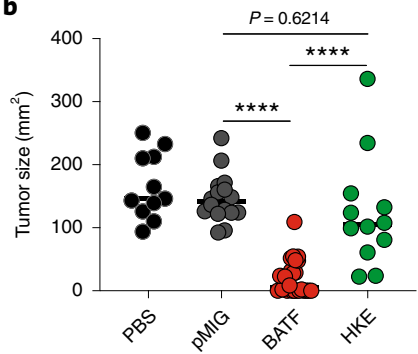

e
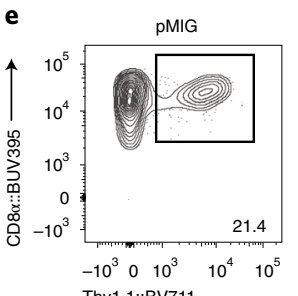

f
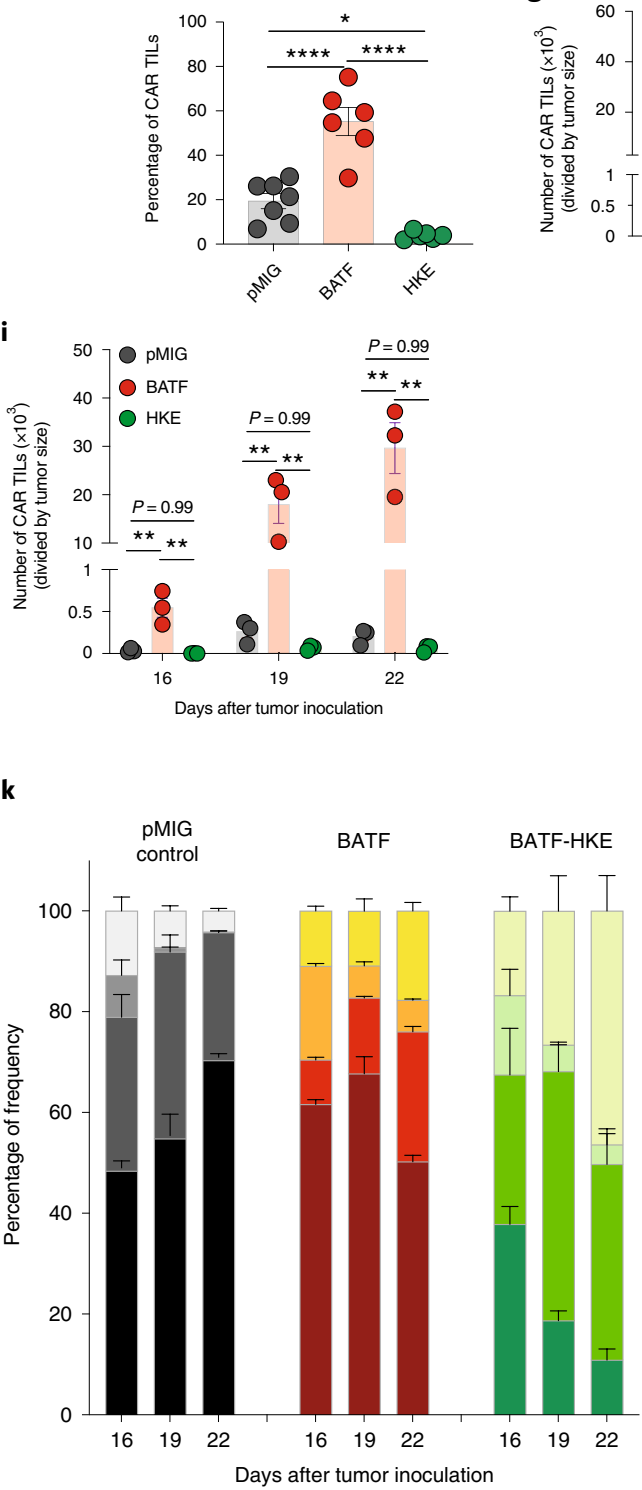

k

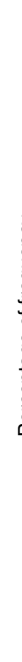

$\mathbf{k}$

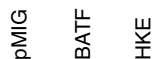

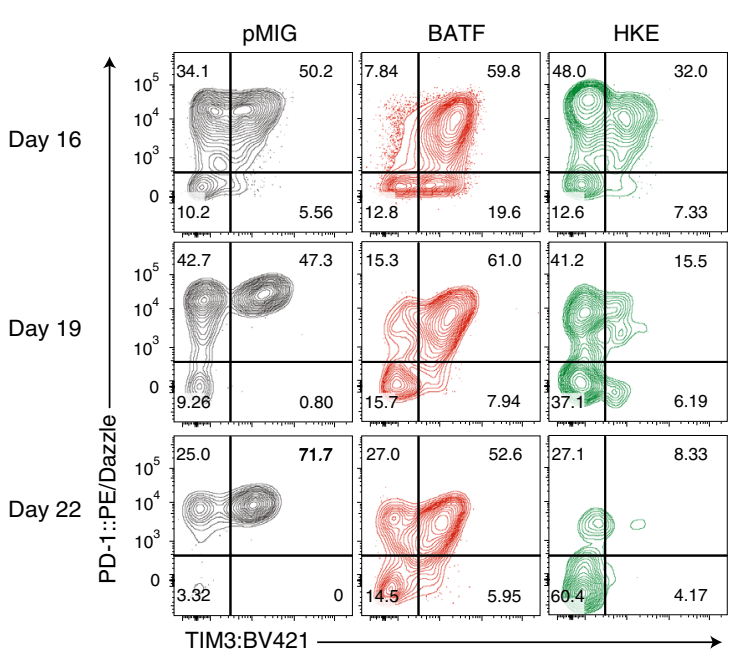

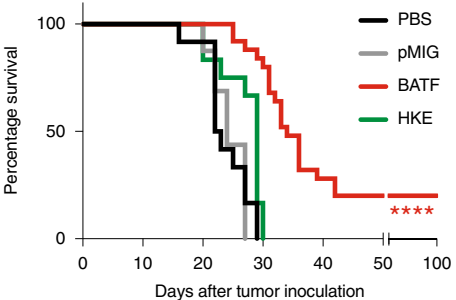
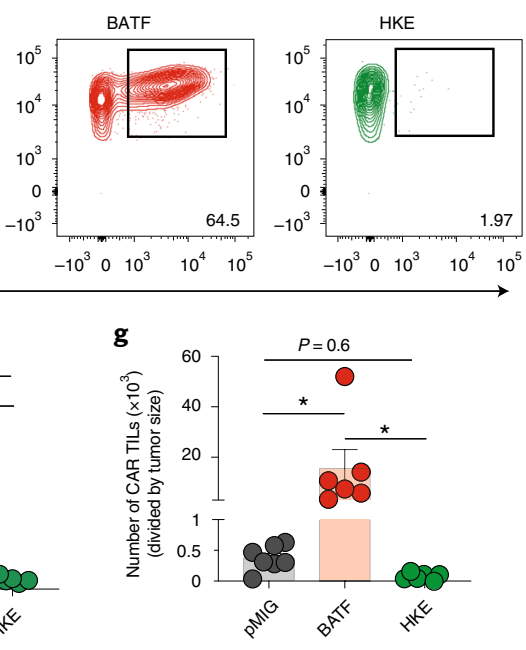

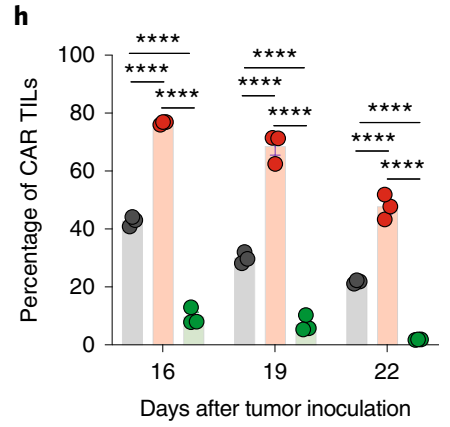

PD-1 ${ }^{-} \mathrm{TIM}^{-}$

PD $-1^{-}$TIM3 $^{+}$

PD $-1^{+} \mathrm{TIM3}^{-}$

$\square \mathrm{PD}-1^{+} \mathrm{TIM3}^{+}$ 
a
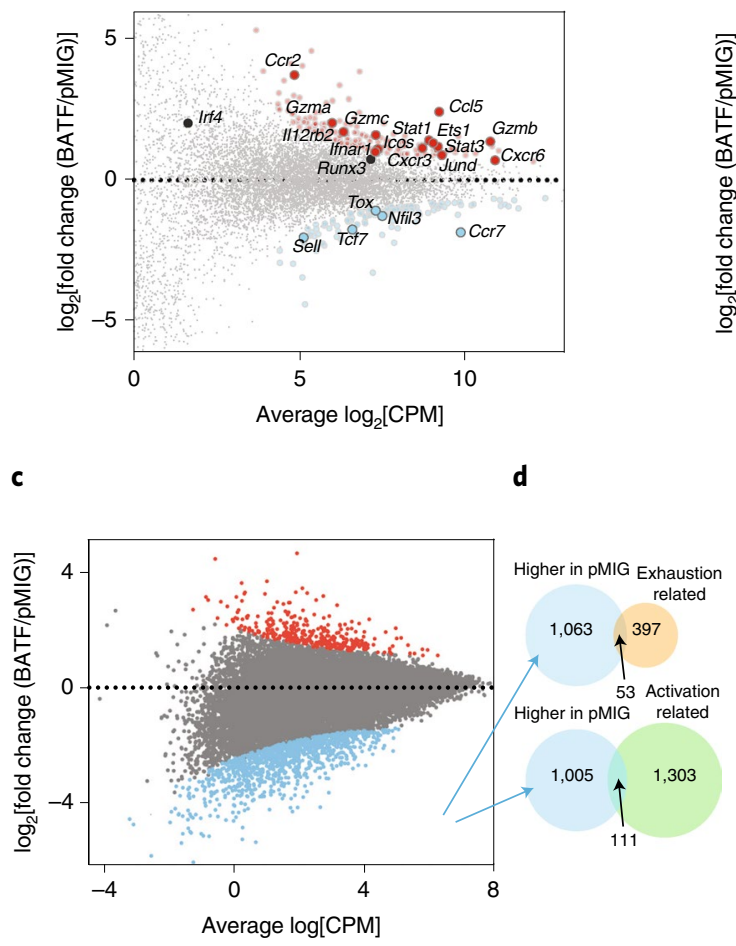

d b

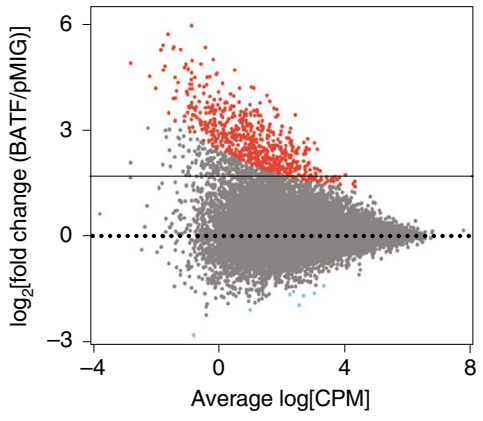

e

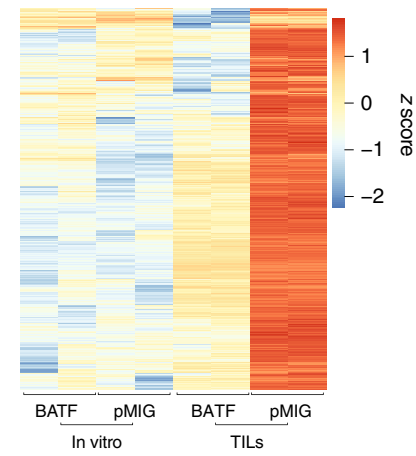

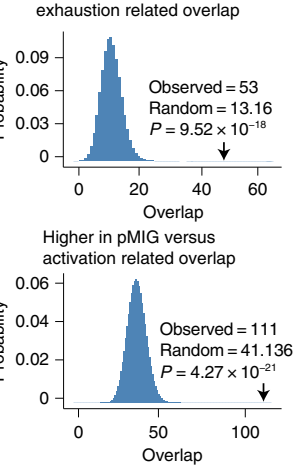

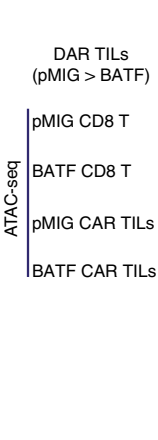

$200 \mathrm{~kb}$

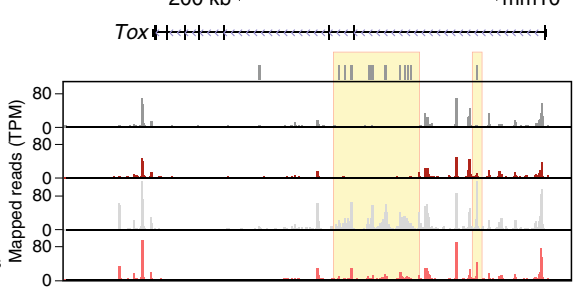

Fig. 5 | Genome-wide analysis of differences in transcription and chromatin accessibility between pMIG- and BATF-transduced cells. a, MA plot of RNA-seq data from BATF-transduced versus pMIG-transduced CAR TILs. Genes differentially expressed in BATF-overexpressing TILs $\left(P_{\text {adj }}<0.1\right.$ and $\log _{2}$ [fold change] $\geq 0.5 ; n=167$ genes $)$ are highlighted in red; genes differentially expressed in pMIG control TILs $\left(P_{\text {adj }}<0.1\right.$ and $\log _{2}$ [fold change] $\leq-0.5$; $n=119$ genes) are highlighted in blue. Selected genes are labeled. b. MA plot of ATAC-seq data from CD8 ${ }^{+}$T cells in vitro, representing 32,035 mapped accessible regions. DARs ( $\log _{2}[$ fold change $] \geq 2$ and $\left.P_{\text {adj }} \leq 0.05\right)$ are plotted as red dots for regions more accessible in BATF-transduced cells $(n=640)$ and blue dots for regions more accessible in pMIG-transduced cells $(n=8)$. Of the 640 regions that were more accessible in BATF-overexpressing cells, 551 overlapped with a BATF ChIP-seq peak. c, MA plot of ATAC-seq data from BATF-transduced versus pMIG-transduced CAR TILs, representing 37,385 mapped accessible regions. DARs ( $\log _{2}\left[\right.$ fold change] $\geq 2$ and $\left.P_{\text {adj }} \leq 0.05\right)$ are plotted as red dots for regions more accessible in BATF-transduced cells $(n=351)$ and blue dots for regions more accessible in pMIG-transduced cells $(n=1,116)$. d, Left: Venn diagrams showing the overlap of the 1,116 regions more accessible in PMIG- versus BATF-transduced TILs with the exhaustion-related (top) or activation-related (bottom) regions from Mognol et al. ${ }^{36}$. Right: histograms illustrating the significance calculation by one-tailed Fisher's exact test. e, Heatmap (z scores) of ATAC-seq signals from BATF- and pMIG-transduced CD8 $8^{+}$cells and CAR TILs for the 1,116 regions more accessible in pMIG TILs compared with BATF TILs. Each column represents a biological replicate. f, Tox locus with normalized ATAC-seq signals for CD8 ${ }^{+}$T cells and CAR TILs. The top track marks DARs (pMIG > BATF) in the TILs. Yellow highlighting calls attention to peaks that differ most strikingly between pMIG and BATF TILs. The data in $\mathbf{a}-\mathbf{c}$, e and $\mathbf{f}$ were obtained from two independent biological experiments.

BATF-overexpressing TILs within the tumor environment, we carried out parallel analyses with the assay for transposase-accessible chromatin using sequencing (ATAC-seq) and RNA sequencing (RNA-seq) on transduced $\mathrm{T}$ cells obtained just before adoptive transfer and on BATF-overexpressing and control TILs harvested from tumors $8 \mathrm{~d}$ after adoptive transfer. The very limited alterations in chromatin accessibility in vitro in BATF-overexpressing cells compared with pMIG cells were strongly biased toward greater accessibility in BATF-overexpressing cells. Of the 32,035 accessible chromatin regions mapped, 640 regions were more accessible in BATF-overexpressing cells and just eight regions were less accessible (Fig. 5b and Supplementary Table 2).

In TILs, in contrast, a solid majority of the differentially accessible regions (DARs) were more accessible in control pMIG TILs than in BATF-overexpressing TILs (Fig. 5c and Supplementary Table 3). The ATAC-seq peak regions showing greater accessibility in pMIG-transduced cells overlapped significantly with both exhaustion- and activation-related peaks identified in TILs by Mognol et al. ${ }^{36}$ (Fig. 5d). (The peaks from Mognol et al. were defined by comparing OT-I tumor antigen-specific $\mathrm{CD}^{+}$cells with P14 bystander $\mathrm{CD}^{+}$cells, and were therefore directly dependent on TCR signaling in the tumor.) In contrast, the regions more accessible in BATF-overexpressing cells were not enriched for the exhaustion-related subset and were depleted for the activation-related subset (Extended Data Fig. 7).

The regions of differentially higher accessibility in pMIG TILs reflected almost exclusively chromatin rearrangements occurring in pMIG TILs after adoptive transfer (Fig. 5e). A telling example is the Tox locus, which exhibited similar accessibility in control pMIGand BATF-transduced cells before adoptive transfer, but showed selective increases in the accessibility of several regions in pMIG TILs (Fig. 5f). The data of Fig. 5d-f indicate that TCR-dependent signals that ordinarily alter the accessibility of characteristic chromatin regions in tumor-responsive $\mathrm{CD}^{+}$TILs are blunted in BATF-overexpressing TILs.

The early patterns of differential accessibility between BATF-expressing and control cells in vitro were superseded by distinct patterns of differential accessibility in TILs (Fig. 5b,c and Extended Data Fig. 7). Binding motifs for ETS, RUNT, bZIP and IRF transcription factors, as well as composite ETS-RUNT and 
a
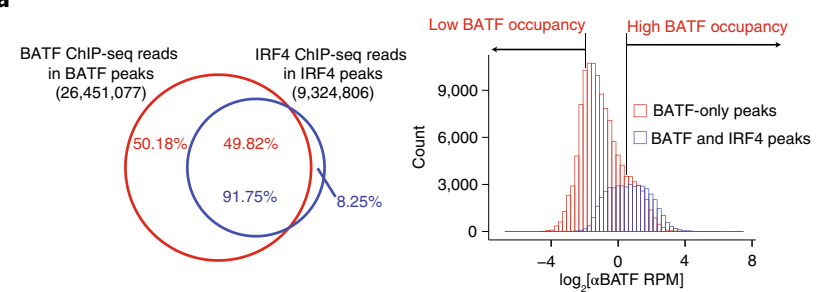

b
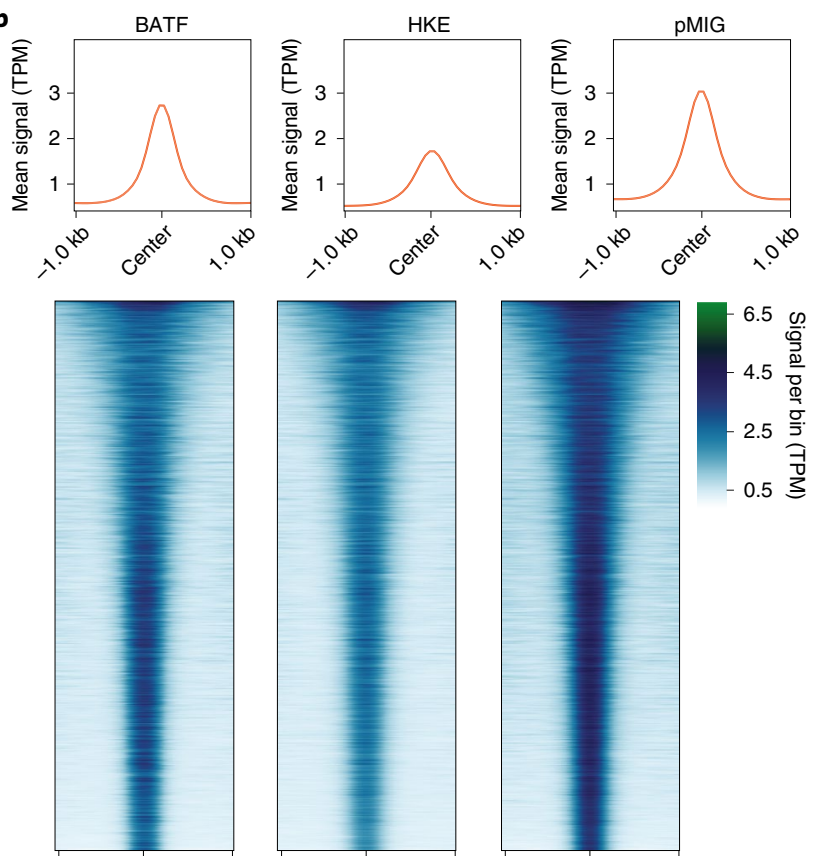

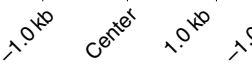
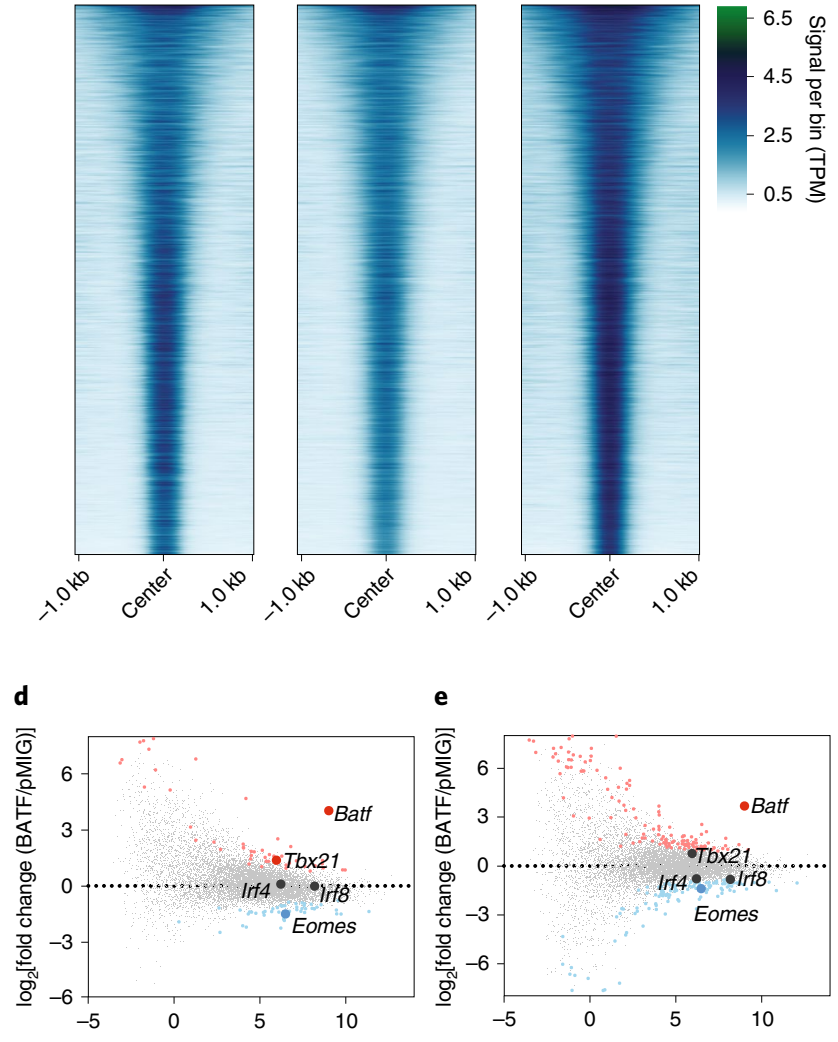

e

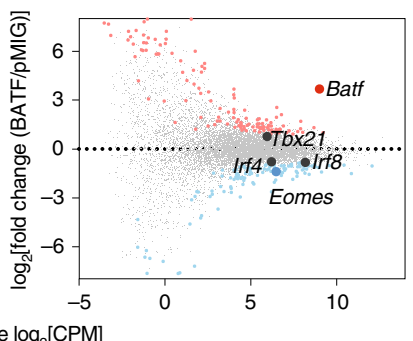

c

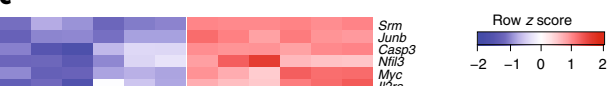

Fig. 6 | BATF and IRF4 binding and gene expression changes in pMIG- and BATF-transduced cells. a, Left: distribution of BATF (red) and IRF4 (blue) ChIP-seq reads in peaks from BATF-transduced cells. Almost all IRF4 reads are located in BATF ChIP-seq peaks, whereas half of BATF reads map to regions that do not coincide with IRF4 peaks. Right: BATF ChIP-seq signal distribution in the shared BATF and IRF4 peaks (blue) and BATF-only peaks (red). Regions in the histogram corresponding to the peaks of high BATF occupancy $\left(\log _{2}[\alpha B A T F R P M]>0.5\right)$ and $\operatorname{low}_{B A T F}$ occupancy $\left(\log _{2}[\alpha B A T F\right.$ RPM] $<-2$ ), as discussed in the text, are indicated. b. Heatmaps of the IRF4 ChIP-seq signal in BATF-, BATF-HKE- and pMIG-transduced cells at IRF4 peak locations called in PMIG-transduced cells. The curves at the top show the average signal taken over all peaks in the respective heatmap. The average signal is modestly decreased in BATF-overexpressing cells compared with pMIG control cells, and substantially reduced in BATF-HKE-overexpressing cells. c, Heatmap of normalized RNA-seq reads (as z scores), under the indicated conditions, for the top 100 genes differentially expressed in pMIG-transduced cells after $\alpha \mathrm{CD} 3 / \alpha \mathrm{CD} 28$ stimulation. d, MA plot of RNA-seq data from BATF-transduced versus pMIG-transduced CD $8^{+} \mathrm{T}$ cells expanded in vitro as for adoptive transfer, without restimulation. Differentially expressed genes more highly expressed in BATF-transduced cells (red dots; $n=47$ ) or pMIG-transduced cells (blue dots; $n=46$ ) are indicated. Selected genes are labeled. e, MA plot of RNA-seq data from BATF-transduced versus pMIG-transduced CD8 ${ }^{+} T$ cells expanded in vitro and restimulated with $\alpha C D 3 / \alpha C D 28$ for $6 \mathrm{~h}$. Differentially expressed genes more highly expressed in BATF-transduced cells (red dots; $n=132$ ) or pMIG-transduced cells (blue dots; $n=130$ ) are indicated. Selected genes are labeled. $\mathbf{f}$, IRF4 (left) and IRF8 (right) expression detected by flow cytometry (MFI) in pMIG- and BATF-transduced CD8 ${ }^{+} \mathrm{T}$ cells that had been expanded in vitro, at the indicated times after restimulation with $\alpha \mathrm{CD} 3 / \alpha C D 28$. The horizontal dashed lines show expression in naive $C D 8^{+} \mathrm{T}$ cells. The data in $\mathbf{a}$ and $\mathbf{b}$ were obtained from two independent biological experiments. The data in c-e were obtained from three independent biological experiments. The data in $\mathbf{f}$ are representative of two independent biological experiments. 
a
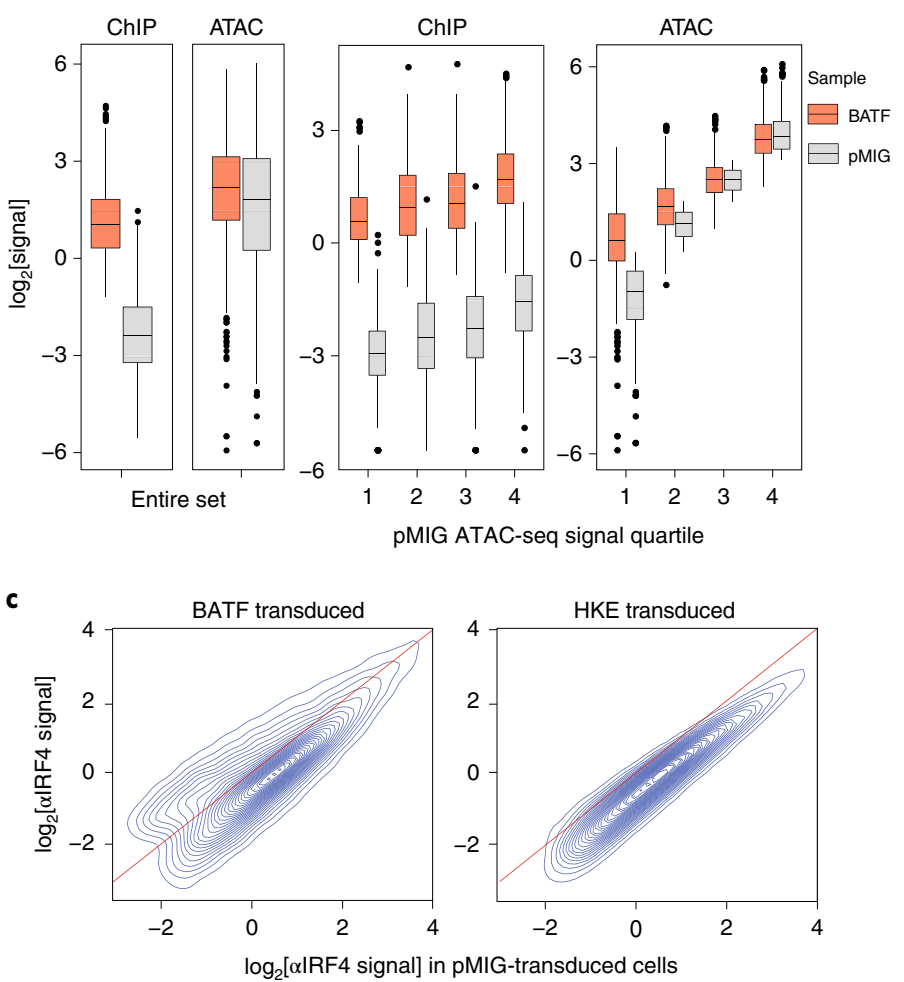

b
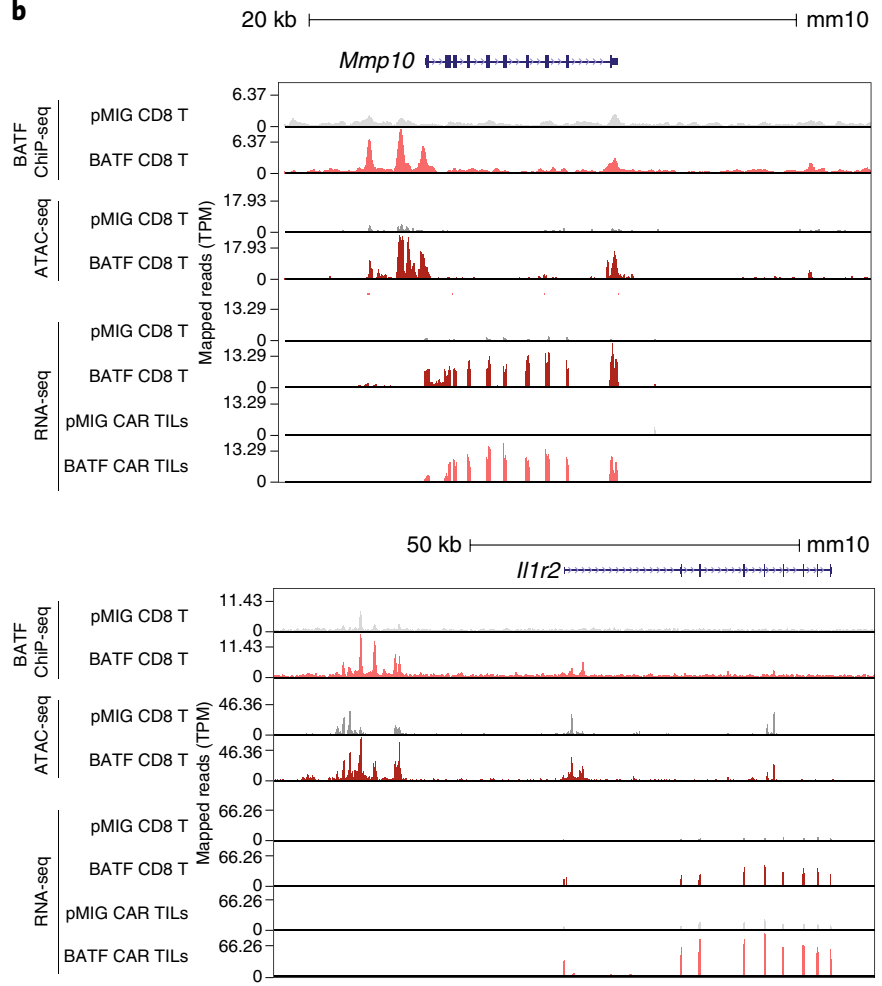

Fig. 7 | Relationship of BATF binding to chromatin accessibility and gene expression in BATF-transduced cells. a, Box and whisker plots showing the distribution of CPM-normalized ATAC-seq and BATF ChIP-seq signals in the collection of BATF ChIP-seq peaks (2,504 peak regions) with a substantial increase in signal ( $\log _{2}[$ fold change $\geq 3$ ) in BATF- compared with pMIG-transduced cells, for the entire set (left) and subdivided into quartiles based on the ATAC-seq signals from pMIG-transduced cells (right). The boxplots represent the minimum values, first quartiles, median values, third quartiles and maximum values of the respective samples, excluding outliers. b. Examples of gene loci where increased BATF binding and increased chromatin accessibility correlate with increased gene expression. Shown are genome browser views of the Mmp10 (top) and II1r2 loci (bottom), with BATF ChIP-seq, ATAC-seq and RNA-seq signals from pMIG- and BATF-transduced CD8 ${ }^{+}$T cells expanded in vitro, as well as RNA-seq signals from pMIG-and BATF-transduced CAR TILs. c, Contour plots relating the IRF4 ChIP-seq signals ( $\log _{2}[C P M]$ ) in BATF-transduced (left) and BATF-HKE-transduced (right) $C D 8^{+} T$ cells to the signals from the corresponding peaks in pMIG-transduced cells. The data in a-c were obtained from two or three independent biological experiments.

bZIP-IRF motifs, were substantially enriched in accessible regions of BATF-overexpressing TILs compared with control TILs (Extended Data Fig. 7). Enrichment of ETS-binding motifs is notable, because Ets1 mRNA was significantly upregulated in BATF-transduced CAR TILs (Fig. 5a); ETS1 contributes to T cell development and homeostatic proliferation, and ETS motifs are enriched in the accessible chromatin of effector/memory $\mathrm{T}$ cells ${ }^{37-39}$, consistent with the ability of BATF-transduced CAR TILs to expand and display effector function.

Early changes in transcription factor binding. ChIP-seq data underline the close cooperation of IRF4 with BATF, since IRF4 binds predominantly at BATF peaks in BATF-overexpressing cells (Fig. 6a). However, the cooperation is not symmetrical, as a large fraction of BATF reads map to peaks where there is no significant IRF4 binding (Fig. 6a). While the majority of these latter peaks have low numbers of reads and may represent nonphysiological binding of BATF, a minor subset shows appreciable BATF occupancy (Fig. 6a, right; shoulder in red histogram). The peaks with highest BATF occupancy, when compared with the peaks with lowest occupancy, are enriched in motifs for ETS family proteins (for example, the HOMER ETS1-binding motif is present in $61.57 \%$ of target sequences and in only $10.59 \%$ of background sequences; $P=1 \times 10^{-3124}$ ) and in motifs comprising short G-rich tracts. Further attention to these peaks is warranted in light of the upregulation of
Ets1 mRNA and the differential enrichment of ETS motifs in accessible regions in BATF-overexpressing CAR TILs.

The IRF4 ChIP-seq profiles at BATF-IRF4 peaks were qualitatively similar in pMIG control cells and BATF-overexpressing cells, implying that endogenous levels of BATF and BATF3 are sufficient to recruit IRF4 in most cases (Fig. 6b). On close examination, though, IRF4 binding at peaks called in pMIG cells was decreased, on average, in BATF-overexpressing cells (Fig. 6b), and IRF4 redistributed within the smaller subset of IRF4 peaks called in BATF-overexpressing cells (Extended Data Fig. 8).

It is known that BATF-HKE can bind adjacent to IRF4 at AICE sites, but that it does not cooperate with IRF4 to stabilize IRF4 binding $^{33}$. In our experiments, despite binding at the same sites as wild-type BATF and increasing total BATF binding (BATF-HKE plus endogenous BATF) at IRF4 peaks over that in PMIG control cells (Extended Data Fig. 8), overexpressed BATF-HKE decreased the average IRF4 signal substantially below the level in control cells (Fig. 6b). The most likely mechanism is competitive displacement of endogenous BATF and BATF3. Correspondingly, gene expression in BATF-HKE-overexpressing cells deviated from the pattern common to BATF-overexpressing and pMIG control cells, especially in unstimulated cells (Extended Data Fig. 8).

Early changes in gene expression. The pattern of gene expression was very similar in BATF- and pMIG-transduced cells in vitro, 
a

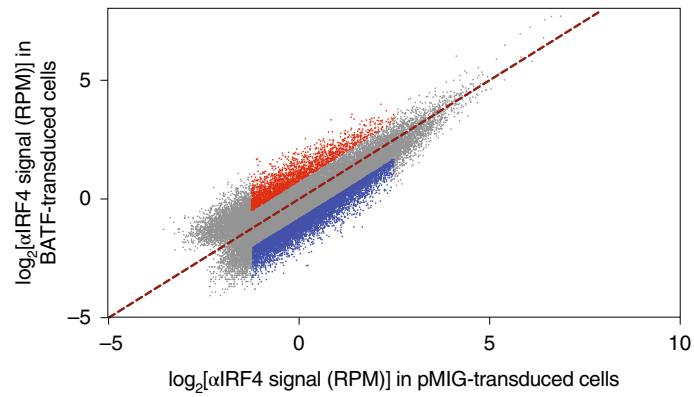

b

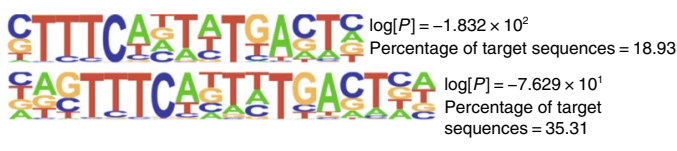

d

\begin{tabular}{|l|c|c|c|c|c|}
\hline \multirow{2}{*}{ Sequence } & \multirow{2}{*}{ Sequence name } & \multicolumn{2}{|c|}{ Increased binding } & \multicolumn{2}{c|}{ Decreased binding } \\
\cline { 3 - 6 } & & $n$ & $\%$ & $n$ & $\%$ \\
\hline GA(T/A)ATGA(G/C)T & AICE2 & 378 & 16.35 & 407 & 4.04 \\
\hline TnnnGA(T/A)ATGA(G/C)T & AICE2 $\left(\right.$ ref. $\left.^{33}\right)$ & 137 & 5.93 & 106 & 1.05 \\
\hline
\end{tabular}

e
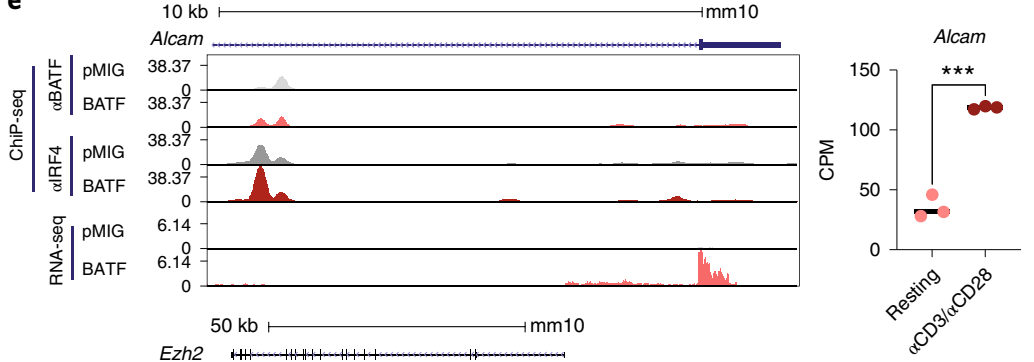

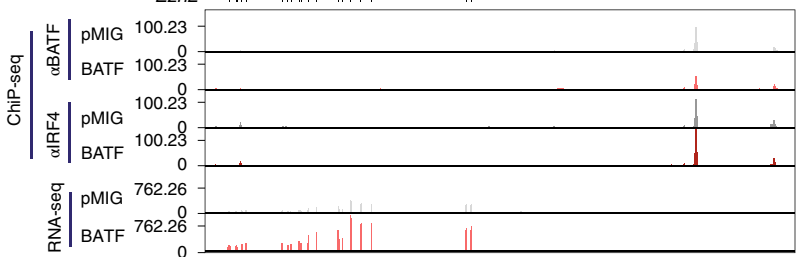

Fig. 8 | Regions of high IRF4 binding in BATF-overexpressing cells are enriched in strong-consensus AICE and AICE2 motifs. a, Dot plot highlighting regions of the IRF4 ChIP-seq data from Fig. 7c (left panel) where IRF4 binding increases ( $\log _{2}[$ fold change $] \geq 0.75 ;$ red dots; $n=2,312$ ) or decreases ( $\log _{2}[$ fold change $] \leq-0.75$; blue dots; $\left.n=10,069\right)$ in BATF-overexpressing relative to $p M I G$-transduced cells. Peaks with very low $\left(\log _{2}[\right.$ signal] $<-1.25$ ) or high ( $\log _{2}[$ signal $>>2.5$ ) IRF4 binding in PMIG cells were judged unlikely to be informative and were omitted from the analysis. The data were obtained from two independent biological experiments. b. Top enriched known motifs reported by HOMER for IRF4 peak regions with increased binding (red dots in a), using as a background the peak regions with decreased binding (blue dots in $\mathbf{a}$ ). c, Top enriched de novo motifs reported by HOMER in the same comparison. d, Number and percentage of peaks in a containing exact matches to strong AICE2 sequences among the 2,312 peaks with increased IRF4 binding and among the 10,069 peaks with decreased IRF4 binding. e, Examples of gene loci where increased IRF4 binding in BATF-overexpressing cells correlates with increased gene expression. Left: genome browser views of Alcam (top) and Ezh2 (bottom) loci, showing BATF ChIP-seq, IRF4 ChIP-seq and RNA-seq signals from pMIG- and BATF-transduced CD8 $8^{+}$T cells. Right: quantification of RNA-seq data for Alcam (top) and Ezh2 (bottom), showing expression changes in opposite directions after stimulation with $\alpha C D 3 / \alpha C D 28$. Each circle represents cells expanded in vitro from one mouse. The data were analyzed by two-tailed unpaired Student's $t$-test. ${ }^{\star \star} P \leq 0.01 ;{ }^{\star \star \star} P \leq 0.001$.

whether considering the subset of mRNAs that exhibited the most significant upregulation or downregulation upon $\alpha \mathrm{CD} 3 / \alpha \mathrm{CD} 28$ stimulation (Fig. 6c) or all mRNAs at rest and upon activation (Fig. 6d,e). The congruence in gene expression is consistent with the similar patterns of chromatin accessibility and similar IRF4 binding at BATF-IRF4 peaks in BATF-overexpressing and control pMIG-transduced cells. The overall similarity does not imply that the patterns of gene expression in BATF-transduced and pMIG-transduced cells are identical (Supplementary Table 4). It is particularly notable that $T b x 21$ (encoding T-bet) is upregulated in BATF-overexpressing cells at the time of adoptive transfer, and Eomes is downregulated, which could well predispose the cells toward effector function and against exhaustion ${ }^{2,40-42}$.

Induction of IRF4 and IRF8 proteins upon stimulation is reduced in BATF-overexpressing cells compared with control cells (Fig. 6f). This is a clear indication that some aspects of TCR signaling have been rewired in BATF-overexpressing cells, and it may have special relevance in light of the finding that high IRF4 expression can antagonize the beneficial effects of BATF on tumor control.

Newly accessible chromatin sites. We asked whether overexpressed BATF might act as a pioneer factor to open new chromatin sites $^{19,22,43,44}$. BATF ChIP-seq peaks with a substantially higher signal in BATF-overexpressing compared with control cells, as a group, do not display correspondingly elevated local chromatin accessibility
(Fig. 7a). However, when the peaks are subgrouped into quartiles based on the ATAC-seq signal in pMIG cells, increased BATF binding in BATF-overexpressing cells is correlated with the opening of chromatin for regions comprising the lowest quartile of ATAC-seq signal (Fig. 7a). Whether BATF binding is causative for increased chromatin accessibility can only be tested directly by engineered mutation of these sites.

Which genes are near these newly accessible sites, and are any of them upregulated? A full list of the neighboring genes is given in Supplementary Table 5. At least some of these genes are upregulated both pretransfer and in TILs (examples are Mmp10 and Il1r2; Fig. 7b), suggesting that increased chromatin accessibility may contribute to increased gene expression in the relatively small number of loci where BATF binding and chromatin accessibility are sharply higher in BATF-overexpressing cells. The main conclusion, though, is that overexpressed BATF binds predominantly within chromatin regions that are accessible in control pMIG cells, comprising regions that were already accessible in naive $\mathrm{CD}^{+} \mathrm{T}$ cells and regions that became accessible when the cells were activated before retroviral transduction.

Redistribution of IRF4 among its binding sites. We established that normalized $\alpha I R F 4$ ChIP-seq reads report accurately on IRF4 binding at individual sites, for comparisons between BATF-overexpressing cells and control cells (Methods and Extended 
Data Fig. 9). Quantitative examination of the data then led to two substantive conclusions. First, echoing the finding for the average IRF4 signal at its peaks in Fig. 6b, IRF4 binding was measurably decreased at most peaks in BATF-overexpressing cells (Fig. 7c, left). Second, there was a redistribution of IRF4 among its binding sites, since IRF4 binding was unchanged or increased at a minority of peaks (Fig. 7c (left) and Extended Data Fig. 9).

The reduced induction of IRF4 in wild-type BATF-overexpressing cells can account for the decrease in IRF4 binding at most peaks, but why was IRF4 binding to certain sites higher in BATF-overexpressing cells than in pMIG cells? Stabilization of IRF4 binding by increased BATF occupancy at AICE sites is probably one contributing factor, and its contribution will vary from site to site, because the binding energies of BATF-IRF4-DNA complexes vary with the DNA sequence at the individual site ${ }^{45}$. In a motif enrichment analysis of peaks that were farther off the diagonal (either above or below) in Fig. $7 \mathrm{c}$ than would be expected just from scatter in the data, the top two known motifs in the set above the diagonal were different versions of the BATF-IRF element AICE (Fig. 8a,b). These motifs were seen in a substantial fraction of the peaks. The top de novo enriched sequence was very similar to the alternative BATFIRF element AICE2, and was detected in nearly half of the peaks (Fig. 8c). As a stringent test, we searched for exact matches to four recognized strong AICE2 sequences ${ }^{45}$, as well as for exact matches to a specialized version of AICE2 (ref. ${ }^{33}$ ). We found massive enrichment for strong AICE2 sequences in the set above the diagonal (Fig. 8d), even though the peaks in the comparison set below the diagonal were also identified as IRF4 binding peaks by ChIP-seq, and most of them overlapped BATF-binding peaks. Consistent with their increased BATF and IRF4 binding, $\alpha$ IRF4 peaks above the diagonal tended to exhibit greater chromatin accessibility in BATF-overexpressing cells (Extended Data Fig. 9). Stabilization of IRF4 binding by neighboring transcription factors that are differentially expressed or differentially activated in BATF-overexpressing cells is another likely contributing factor and will also depend on the local DNA context.

BATF-HKE-overexpressing cells showed a consistent decrease in IRF4 binding at individual peaks, which was not due to reduced IRF4 protein (Extended Data Fig. 9), and no redistribution of IRF4 (Fig. 7c (right) and Extended Data Fig. 9). The major factor affecting IRF4 binding in BATF-HKE-overexpressing cells is likely to be the replacement of endogenous BATF and BATF3 at AICE sites by BATF-HKE, resulting in a lower affinity for IRF4. The consistent decrease in IRF4 binding elicited by BATF-HKE overexpression is compelling evidence that nearly all IRF4 binding in pMIG control cells depends on the interaction with BATF.

IRF4 binding and gene expression. The evidence indicates that IRF4 binding is tempered by other inputs in determining gene expression. Alcam and Ezh2 are known BATF-IRF4 target genes that exhibit both enhanced IRF4 binding and significantly higher expression in BATF-overexpressing cells (Fig. 8e, left), but in both cases mRNA levels change appreciably and in opposite directions upon $\alpha \mathrm{CD} 3 / \alpha \mathrm{CD} 28$ stimulation, indicating that other transcription factors have a role in determining the transcriptional output (Fig. 8e, right). Moreover, the quantitative changes in IRF4 binding in BATF-overexpressing cells are in general small (the shift in the modal value is $\sim 0.4 \log _{2}$ units over a broad range of ChIP-seq signals in pMIG cells, which translates to a decrease of $~ 25 \%$ in bound IRF4) and the extent of variability is restricted in most cases to a range of one $\log _{2}$ unit around the modal value (Fig. 7c). We propose that alterations in IRF4 binding may predominate in controlling the transcriptional output in some cases, while in other cases IRF4 binding only sets a bias, and other transcription factors whose levels or activities differ between BATF-overexpressing and pMIG cells determine the final output.

\section{Discussion}

The progressive development of $\mathrm{CD}^{+} \mathrm{T}$ cell exhaustion in TILs and during chronic viral infection occurs through the concerted actions of transcription factors, which impose exhaustion through changes in chromatin structure and gene transcription. One approach to defeating exhaustion is to interfere with the transcription factors that drive it, and we and others have demonstrated that depletion of NR4A or TOX transcription factors (two downstream targets of NFAT that are induced by NFAT and cooperate functionally with NFAT to drive $\mathrm{CD}^{+} \mathrm{T}$ cell exhaustion) allows CD8 ${ }^{+}$TILs to maintain robust effector function ${ }^{4-10}$. Here, we approach the same objective from a different angle, by asking whether the onset of exhaustion might be prevented by maintaining the expression of transcription factors that favor full $\mathrm{T}$ cell effector function. We show that overexpressing BATF in CD8 ${ }^{+}$CAR TILs confers enhanced effector function and robust antitumor responses and prevents the progressive exhaustion that would otherwise occur in the tumor environment. Notably, some BATF-transduced CAR T cells remain after tumor clearance as memory-like cells that are fully capable of making a subsequent antitumor response. Thus, BATF overexpression corrects the two cardinal features of $\mathrm{T}$ cell exhaustion: the immediate limitation on effector function and the long-term limitation on memory formation.

Elements influencing BATF overexpression-induced CD8 ${ }^{+}$TIL function are the early differential expression of Tbx21, Eomes and other key genes in the $\mathrm{T}$ cells before adoptive transfer; alterations in signaling leading to less upregulation of IRF4 in response to TCR stimulation; consequent redistribution of IRF4 among its target sites in chromatin; blunted TCR signaling to chromatin in the tumor, with failure to open many exhaustion-related chromatin regions that normally become accessible in CD8 ${ }^{+}$TILs; and failure of the sustained upregulation of Tox that ordinarily occurs in the tumor.

The observed redeployment of IRF4, and the observed decreased IRF4 binding at many sites, are at first counterintuitive. Overexpressed BATF would ordinarily favor increased IRF4 binding at all BATF-IRF sites, except at sites that were fully occupied in pMIG cells. However, because of altered signaling, IRF4 levels are lower in restimulated BATF-overexpressing cells than in restimulated control cells. When IRF4 is limiting, IRF4 binds preferentially to the higher-affinity sites at the expense of lower-affinity sites, parallel to what was shown for BATF-IRF binding in $\mathrm{CD}^{+} \mathrm{T}$ cells subjected to brief or weak stimulation ${ }^{33}$.

The heightened effector response of BATF-transduced cells depends on BATF-IRF interaction. Previous work in type $2 \mathrm{~T}$ helper $\left(\mathrm{T}_{\mathrm{H}} 2\right)$ and $\mathrm{T}_{\mathrm{H}} 17$ cells established the importance of a subset of BATF sites in DNA, termed AICEs, where JUN-BATF, JUNB-BATF or JUND-BATF heterodimers bind in a complex with IRF4 or IRF8 (refs. ${ }^{20,23,24,33}$ ). The recruitment of IRF4 to these AICE sites is substantially weakened by the HKE substitutions in BATF, and the HKE substitutions are known to compromise IRF4-mediated transcription in $\mathrm{T}_{\mathrm{H}} 2$ and $\mathrm{T}_{\mathrm{H}} 17$ cells $^{20,21,23,33}$. In our study, CD8 ${ }^{+}$CAR TILs overexpressing the BATF-HKE variant failed to survive and expand in tumors, consistent with the known requirements for BATF and IRF4 in early effector CD8 ${ }^{+}$T cell expansion ${ }^{19,46}$.

BATF and IRF4 are both induced by TCR activation, and there is ample evidence that BATF and IRF4 are essential for metabolic reprogramming and clonal expansion of effector $\mathrm{CD}^{+} \mathrm{T}$ cells $\mathrm{s}^{19,25,46,47}$. The modest upregulation of BATF in chronic viral infections and certain other observations led to the view that BATF and IRF4 might help to induce $\mathrm{T}$ cell exhaustion ${ }^{25,26}$. However, another report for chronic lymphocytic choriomeningitis virus clone 13 infection closely paralleled our findings, demonstrating that overexpressing BATF in virus-specific P14 TCR-transgenic CD8 ${ }^{+} \mathrm{T}$ cells increased their proliferation, expression of effector markers and control of the viral infection ${ }^{48}$. The straightforward interpretation of these varied findings is that BATF and IRF4, like NFAT, are ambivalent 
transcription factors that can contribute to either effector or exhaustion programs in $\mathrm{CD}^{+} \mathrm{T}$ cells depending on the signaling context.

In summary, engineered expression of BATF at high levels supports effective antitumor responses in $\mathrm{CD}^{+} \mathrm{T}$ cells. BATF overexpression yielded CAR TILs that were skewed towards an effector phenotype, underwent striking expansion in tumors, secreted large amounts of effector cytokines and expressed decreased amounts of TOX (a transcription factor notably associated with exhaustion). Additional experiments will be needed to completely elucidate the transcriptional networks involved. From a therapeutic point of view, however, BATF overexpression in CAR TILs has a markedly beneficial effect on both immediate and long-term antitumor responses, since it promotes the formation of long-lived memory cells that can control tumor recurrence.

\section{Online content}

Any methods, additional references, Nature Research reporting summaries, source data, extended data, supplementary information, acknowledgements, peer review information; details of author contributions and competing interests; and statements of data and code availability are available at https://doi.org/10.1038/ s41590-021-00964-8.

Received: 20 July 2020; Accepted: 20 May 2021;

Published online: 19 July 2021

\section{References}

1. Blank, C. U. et al. Defining 'T cell exhaustion'. Nat. Rev. Immunol. 19, 665-674 (2019).

2. McLane, L. M., Abdel-Hakeem, M. S. \& Wherry, E. J. CD8 T cell exhaustion during chronic viral infection and cancer. Annu. Rev. Immunol. 37, 457-495 (2015).

3. Pereira, R. M., Hogan, P. G., Rao, A. \& Martinez, G. J. Transcriptional and epigenetic regulation of $\mathrm{T}$ cell hyporesponsiveness. J. Leukoc. Biol. 102, 601-615 (2017).

4. Liu, X. et al. Genome-wide analysis identifies NR4A1 as a key mediator of T cell dysfunction. Nature 567, 525-529 (2019).

5. Chen, J. et al. NR4A transcription factors limit CAR T cell function in solid tumours. Nature 567, 530-534 (2019).

6. Seo, H. et al. TOX and TOX2 transcription factors cooperate with NR4A transcription factors to impose $\mathrm{CD} 8^{+} \mathrm{T}$ cell exhaustion. Proc. Natl Acad. Sci. USA 116, 12410-12415 (2019).

7. Scott, A. C. et al. TOX is a critical regulator of tumour-specific T cell differentiation. Nature 571, 270-274 (2019).

8. Wang, X. et al. TOX promotes the exhaustion of antitumor $\mathrm{CD} 8^{+} \mathrm{T}$ cells by preventing PD1 degradation in hepatocellular carcinoma. J. Hepatol. 71, 731-741 (2019).

9. Alfei, F. et al. TOX reinforces the phenotype and longevity of exhausted $\mathrm{T}$ cells in chronic viral infection. Nature 571, 265-269 (2019).

10. Khan, O. et al. TOX transcriptionally and epigenetically programs $\mathrm{CD} 8^{+}$ $\mathrm{T}$ cell exhaustion. Nature 571, 211-218 (2019).

11. Macián, F. et al. Transcriptional mechanisms underlying lymphocyte tolerance. Cell 109, 719-731 (2002).

12. Martinez, G. J. et al. The transcription factor NFAT promotes exhaustion of activated CD8 ${ }^{+} \mathrm{T}$ cells. Immunity 42, 265-278 (2015).

13. Hogan, P. G., Chen, L., Nardone, J. \& Rao, A. Transcriptional regulation by calcium, calcineurin, and NFAT. Gene Dev. 17, 2205-2232 (2003).

14. Karin, M., Liu, Z. \& Zandi, E. AP-1 function and regulation. Curr. Opin. Cell Biol. 9, 240-246 (1997).

15. Oh-hora, M. et al. Dual functions for the endoplasmic reticulum calcium sensors STIM1 and STIM2 in T cell activation and tolerance. Nat. Immunol. 9, 432-443 (2008)

16. Scott-Browne, J. P. et al. Dynamic changes in chromatin accessibility occur in $\mathrm{CD}^{+} \mathrm{T}$ cells responding to viral infection. Immunity 45, 1327-1340 (2016).

17. Hogan, P. G. Calcium-NFAT transcriptional signalling in T cell activation and $\mathrm{T}$ cell exhaustion. Cell Calcium 63, 66-69 (2017).

18. Lynn, R. C. et al. c-Jun overexpression in CAR T cells induces exhaustion resistance. Nature 576, 293-300 (2019).

19. Kurachi, M. et al. The transcription factor BATF operates as an essential differentiation checkpoint in early effector $\mathrm{CD} 8^{+} \mathrm{T}$ cells. Nat. Immunol. 15, 373-383 (2014).
20. Murphy, T. L., Tussiwand, R. \& Murphy, K. M. Specificity through cooperation: BATF-IRF interactions control immune-regulatory networks. Nat. Rev. Immunol. 13, 499-509 (2013).

21. Li, P. et al. BATF-JUN is critical for IRF4-mediated transcription in T cells. Nature 490, 543-546 (2012).

22. Ciofani, M. et al. A validated regulatory network for $\mathrm{T}_{\mathrm{H}} 17$ cell specification. Cell 151, 289-303 (2012).

23. Glasmacher, E. et al. A genomic regulatory element that directs assembly and function of immune-specific AP-1-IRF complexes. Science 338, 975-980 (2012).

24. Tussiwand, R. et al. Compensatory dendritic cell development mediated by BATF-IRF interactions. Nature 490, 502-507 (2012).

25. Man, K. et al. Transcription factor IRF4 promotes $\mathrm{CD} 8^{+} \mathrm{T}$ cell exhaustion and limits the development of memory-like $\mathrm{T}$ cells during chronic infection. Immunity 47, 1129-1141.e5 (2017).

26. Quigley, M. et al. Transcriptional analysis of HIV-specific $\mathrm{CD} 8^{+} \mathrm{T}$ cells shows that PD-1 inhibits T cell function by upregulating BATF. Nat. Med. 16, 1147-1151 (2010).

27. Im, S. J. et al. Defining $\mathrm{CD} 8^{+} \mathrm{T}$ cells that provide the proliferative burst after PD-1 therapy. Nature 537, 417-421 (2016).

28. Kurtulus, S. et al. Checkpoint blockade immunotherapy induces dynamic changes in PD- $1^{-} \mathrm{CD} 8^{+}$tumor-infiltrating T cells. Immunity $\mathbf{5 0}$, 181-194.e6 (2019).

29. Siddiqui, I. et al. Intratumoral $\mathrm{Tcf} 1^{+} \mathrm{PD}-1^{+} \mathrm{CD} 8^{+} \mathrm{T}$ cells with stem-like properties promote tumor control in response to vaccination and checkpoint blockade immunotherapy. Immunity 50, 195-211.e10 (2019).

30. Miller, B. C. et al. Subsets of exhausted $\mathrm{CD}^{+} \mathrm{T}$ cells differentially mediate tumor control and respond to checkpoint blockade. Nat. Immunol. 20, 326-336 (2019).

31. Utzschneider, D. T. et al. T cell factor 1-expressing memory-like CD8 ${ }^{+} \mathrm{T}$ cells sustain the immune response to chronic viral infections. Immunity 45 , 415-427 (2016)

32. Brogdon, J., June, C. H., Loew, A., Maus, M. \& Scholler, J. Treatment of cancer using humanized anti-CD19 chimeric antigen receptor. WO patent WO2014153270A1 (2014).

33. Iwata, A. et al. Quality of TCR signaling determined by differential affinities of enhancers for the composite BATF-IRF4 transcription factor complex. Nat. Immunol. 18, 563-572 (2017).

34. Xiao, Z., Casey, K. A., Jameson, S. C., Curtsinger, J. M. \& Mescher, M. F. Programming for CD8 T cell memory development requires IL-12 or type I IFN. J. Immunol. 182, 2786-2794 (2009).

35. Huber, J. P. \& Farrar, J. D. Regulation of effector and memory T-cell functions by type I interferon. Immunology 132, 466-474 (2011).

36. Mognol, G. P. et al. Exhaustion-associated regulatory regions in CD8 ${ }^{+}$ tumor-infiltrating T cells. Proc. Natl Acad. Sci. USA 114, E2776-E2785 (2017).

37. Jadhav, R. R. et al. Epigenetic signature of PD- $1^{+} \mathrm{TCF} 1^{+} \mathrm{CD} 8 \mathrm{~T}$ cells that act as resource cells during chronic viral infection and respond to PD-1 blockade. Proc. Natl Acad. Sci. USA 116, 14113-14118 (2019).

38. Muthusamy, N., Barton, K. \& Leiden, J. M. Defective activation and survival of T cells lacking the Ets-1 transcription factor. Nature 377, 639-642 (1995).

39. Grenningloh, R. et al. Ets-1 maintains IL-7 receptor expression in peripheral T cells. J. Immunol. 186, 969-976 (2011).

40. Wherry, E. J. T cell exhaustion. Nat. Immunol. 12, 492-499 (2011).

41. Wherry, E. J. \& Kurachi, M. Molecular and cellular insights into T cell exhaustion. Nat. Rev. Immunol. 15, 486-499 (2015).

42. Li, J., He, Y., Hao, J., Ni, L. \& Dong, C. High levels of Eomes promote exhaustion of anti-tumor $\mathrm{CD} 8^{+} \mathrm{T}$ cells. Front. Immunol. 9, 2981 (2018).

43. Li, P. \& Leonard, W. J. Chromatin accessibility and interactions in the transcriptional regulation of T cells. Front. Immunol. 9, 2738 (2018).

44. Pham, D. et al. Batf pioneers the reorganization of chromatin in developing effector T cells via Ets1-dependent recruitment of Ctcf. Cell Rep. 29, 1203-1220.e7 (2019).

45. Chang, Y. K., Zuo, Z. \& Stormo, G. D. Quantitative profiling of BATF family proteins/JUNB/IRF hetero-trimers using Spec-seq. BMC Mol. Biol. 19, 5 (2018).

46. Man, K. et al. The transcription factor IRF4 is essential for TCR affinity-mediated metabolic programming and clonal expansion of $\mathrm{T}$ cells. Nat. Immunol. 14, 1155-1165 (2013).

47. Wei, J. et al. Targeting REGNASE-1 programs long-lived effector $\mathrm{T}$ cells for cancer therapy. Nature 576, 471-476 (2019).

48. Xin, G. et al. A critical role of IL-21-induced BATF in sustaining CD8-T-cell-mediated chronic viral control. Cell Rep. 13, 1118-1124 (2015).

Publisher's note Springer Nature remains neutral with regard to jurisdictional claims in published maps and institutional affiliations.

๑ The Author(s), under exclusive licence to Springer Nature America, Inc. 2021 


\section{Methods}

This research complies with all relevant ethical regulations.

Approval for vertebrate animal studies was provided by the La Jolla Institute for Immunology (LJI) Animal Care Committee (protocol number AP00001025).

Approval for human studies was provided by the LJI Institutional Review Board (protocol number SCRO_002). Informed consent was obtained from all human blood donors. Donors were compensated per LJI policy.

Mice. C57BL6/J, B6.SJL-Ptprc ${ }^{\mathrm{a}}$ Pepc ${ }^{\mathrm{b}} /$ BoyJ (CD45.1) and C57BL/6-Tg(TcraTcrb) $1100 \mathrm{Mjb} / \mathrm{J}(\mathrm{OT}-\mathrm{I})$ mice were obtained from The Jackson Laboratory. CD45.1 ${ }^{+} \mathrm{OT}-\mathrm{I}$ mice were obtained by crossbreeding. Male mice were used for the experiments on antitumor effects in vivo, and both male and female mice were used for the in vitro studies. Six-week-old mice were purchased from The Jackson Laboratory to be used as recipient mice, and rested for at least 1 week after delivery before they were used for the experiments. Mice were age matched and between 7 and 12 weeks old when they were used for the experiments. All mice were bred and/or managed in the animal facility at the LJI. All experiments were performed in compliance with study protocol number AP00001025 approved by the LJI Animal Care Committee. The approved protocol specified a maximum tumor size of $1.77 \mathrm{~cm}^{3}$ or greater for $3 \mathrm{~d}$ without signs of regression. This maximum tumor size was not exceeded.

Human peripheral $\mathrm{CD8}^{+} \mathrm{T}$ cell isolation. Whole blood samples from healthy subjects were collected by a staff phlebotomist in the Clinical Studies Core at the LJI, and peripheral blood mononuclear cells were isolated using Lymphoprep (STEMCELL Technologies) according to the manufacturer's protocol. Peripheral $\mathrm{CD}^{+} \mathrm{T}$ cells were negatively isolated using a human $\mathrm{CD}^{+} \mathrm{T}$ cell isolation kit (STEMCELL Technologies) following the manufacturer's protocol.

Construction of retroviral and lentiviral vectors. CAR expression plasmid. The sequence of the retroviral vector (MSCV-myc-CAR-2A-Thy1.1) encoding the Myc epitope-tagged CAR has been reported previously ${ }^{49,50}$. It contains the hCD19 single-chain variable fragment ${ }^{49}$ and the murine $\mathrm{CD} 3 \zeta$ and $\mathrm{CD} 28$ sequences. The CAR complementary DNA was cloned into an MSCV-puro murine retroviral vector in place of PGK-puro.

Human CD19 retroviral expression plasmid. A PCR-amplified DNA fragment encoding hCD19 was cloned into an MSCV-puro (Clontech) murine retroviral vector as described in previous papers ${ }^{5,6}$.

Retroviral vectors (MSCV-bZIP-IRES-Thy1.1 and MSCV-bZIP-IRES-eGFP). To generate pMIG-Batf, the Batf coding sequence was amplified from pMSCV-Batf-IRES-Thy1.1 (C.-W.J.L.; unpublished), derived from pcDNA3.1-Batf (Addgene; 34575), and cloned into pMSCV-IRES-eGFP (Addgene; 27490). DNA fragments encoding Jun, Maff and the Batf HKE mutant were PCR amplified or synthesized as gBlocks (Integrated DNA Technologies) and cloned into MSCV-IRES-eGFP (Addgene plasmid 27490), kindly provided by W. S. Pear (Univ. Pennsylvania). pMIG-IRF4 was purchased from Addgene (58987)

Lentiviral vectors ( $p$ TRPE-19.28z-P2A-NGFR, $p$ TRPE-IRES-eGFP and pTRPE-BATF-IRES-eGFP). The plasmid pTRPE-19.28z, which contains the hCD19 single-chain variable fragment and the human CD3 $\zeta$ and CD28 sequences, was kindly provided by A. D. Posey Jr (University of Pennsylvania). A fragment containing the P2A and nerve growth factor receptor (NGFR) sequences was PCR amplified and cloned into the pTRPE- $19.28 \mathrm{z}$ vector to yield $\mathrm{pTRPE}$ 19.28z-P2A-NGFR. A fragment containing the internal ribosome entry site (IRES) and enhanced green fluorescent protein (eGFP) sequences was PCR amplified and cloned into the pTRPE-19.28z vector in place of $19.28 \mathrm{z}$ to yield pTRPE-IRES-eGFP. DNA fragments encoding human BATF were synthesized as gBlocks (Integrated DNA Technologies) and cloned into pTRPE-IRES-eGFP.

Cloning of NFAT-AP-1 reporter plasmids. A retroviral reporter plasmid containing six tandem NFAT-AP-1 sites driving GFP expression on a self-inactivating retroviral backbone was kindly provided by H. Spits ${ }^{51}$. Mouse Thyl.1 was cloned into this plasmid, in place of the GFP reporter, using Gibson Assembly. The mouse genes for Jun, Maff, Batf, Batf3, Jund, Fosl2 and Nfil3 were synthesized as gBlocks (Integrated DNA Technologies) and cloned downstream of Thy1.1 with a P2A linker in between using Gibson Assembly.

Cell lines. The B16F0 mouse melanoma cell line was purchased from the American Type Culture Collection. The B16F0-hCD19 cell line was generated by transduction with amphotropic virus encoding hCD19, followed by sorting for cells expressing high levels of hCD19. The B16F10-OVA mouse melanoma cell line was kindly provided by S. Schoenberger (LJI). The Platinum-E Retroviral Packaging Cell Line, Ecotropic (Plat-E) cell line was purchased from Cell Biolabs. All tumor cell lines were tested frequently to be sure they were negative for Mycoplasma contamination and were used at passage 4 after thawing from stock.
Transfections. A total of $3 \times 10^{6}$ Plat-E cells were seeded in $10 \mathrm{~cm}$ dishes in media (Dulbecco's modified Eagle's medium with 10\% fetal bovine serum (FBS), 1\% L-glutamine and $1 \%$ penicillin/streptomycin) the day before transfection, and the medium was changed just before transfection. For retroviral transduction, we used a mixture of $10 \mu \mathrm{g}$ retroviral plasmid $+3.4 \mu \mathrm{g}$ pCL-Eco packaging vectors or PCL10A1. For lentiviral transduction, the mixture contained $10 \mu \mathrm{g}$ lentiviral plasmid $+7.5 \mu \mathrm{g} \mathrm{Gag} \mathrm{pol}+5 \mu \mathrm{g}$ Rev $+2.5 \mu \mathrm{g}$ VSV-G packaging vectors. The plasmid mixtures were incubated with $40 \mu \mathrm{l}$ TransIT-LT1 Transfection Reagent (Mirus Bio) at $\sim 22^{\circ} \mathrm{C}$ for $20 \mathrm{~min}$ in $1.5 \mathrm{ml}$ Opti-MEM media and then added to the Plat-E cells, after which the cells were incubated at $37^{\circ} \mathrm{C}$ in a $10 \% \mathrm{CO}_{2}$ incubator for $30-40 \mathrm{~h}$. The supernatant was filtered through a $40-\mu \mathrm{m}$ filter before being used for transduction of $\mathrm{CD}^{+} \mathrm{T}$ cells

Tumor experiments. Preparation of B16F0-hCD19 and B16F10-OVA melanoma cells for tumor inoculation. Tumor cells (B16F0-hCD19 or B16F10-OVA) were thawed and cultured in Dulbecco's modified Eagle's medium with 10\% FBS, $1 \%$ L-glutamine and $1 \%$ penicillin/streptomycin at $37^{\circ} \mathrm{C}$ in a $5 \% \mathrm{CO}_{2}$ incubator, and were split and passaged at days 1,3 and 5 after thawing before inoculation. At the time of tumor inoculation (day 0), cells were trypsinized and resuspended in PBS solution, then injected subcutaneously into 7 - to 12 -week-old C57BL/6J mice.

Generation and transfer of CAR T cells. Splenic CD8 ${ }^{+} \mathrm{T}$ cells from C57BL/6, B6.SJL-Ptprc ${ }^{\mathrm{a}} P e p c^{\mathrm{b}} /$ BoyJ, C57BL/6-Tg(Tcr $\left.\alpha \mathrm{Tcr} \beta\right) 1100 \mathrm{Mjb} / \mathrm{J}$ or CD45.1xOT-I mice were isolated by negative selection using a CD8 isolation kit (Invitrogen or STEMCELL Technologies), activated with $1 \mu \mathrm{g} \mathrm{ml}^{-1}$ anti-CD3 and anti-CD28 for $1 \mathrm{~d}$, then removed from the plates and retrovirally transduced using $15 \mu \mathrm{g} \mathrm{ml}^{-1}$ polybrene at $37^{\circ} \mathrm{C}$, followed by centrifugation at $2,000 \mathrm{~g}$ for $1-2 \mathrm{~h}$. After transduction, cells were cultured in house-made $\mathrm{T}$ cell medium containing $100 \mathrm{U} \mathrm{ml}^{-1}$ human IL-2. A second transduction was performed the next day using the same protocol, after which cells were cultured in $\mathrm{T}$ cell media containing $100 \mathrm{U} \mathrm{ml}^{-1}$ human IL-2 for $3 \mathrm{~d}$. On the day of adoptive transfer, the cells were analyzed by flow cytometry to check the transduction efficiency (typically $90 \%$ for single retroviral transduction and $80 \%$ for double retroviral transductions), and cell counts were obtained using the Accuri flow cytometer. Cells were washed with PBS and resuspended in PBS before adoptive transfer into recipient mice.

Assessing antitumor responses. On day 0, 7- to 12-week-old C57BL/6 $\mathrm{J}$ mice were injected subcutaneously with $1 \times 10^{5} \mathrm{~B} 16 \mathrm{~F} 0$-hCD19 cells or $2.5 \times 10^{5}$ B16F10-OVA cells. When the tumors were palpable, tumor measurements were recorded with a caliper three to four times a week and the tumor size was calculated in $\mathrm{mm}^{2}$ (length $\times$ width). On day $7,3 \times 10^{6} \mathrm{CAR} \mathrm{T}$ cells or $1 \times 10^{6} \mathrm{OT}-\mathrm{I}$ $\mathrm{T}$ cells were adoptively transferred into tumor-bearing mice. For all survival experiments, tumor growth was monitored until an experimental endpoint of day 100 after tumor inoculation or until the Institutional Animal Care and Use Committee-approved endpoint of a maximum tumor size measurement exceeding a diameter of $1.77 \mathrm{~cm}^{3}$ for more than $3 \mathrm{~d}$ without signs of regression. If mice were pale, had scars or ulcerations or adopted a hunched position, or if their body temperature was low, we euthanized the mice under the guidance of the staff of the Department of Laboratory Animal Care (DLAC) at the LJI. In most cases, tumor sizes were measured in a blinded manner by DLAC staff, except during the holiday season or when the institute was under restricted access due to the COVID-19 shutdown

Harvesting TILs. On day 0, 7- to 12-week-old C57BL/6J mice were injected subcutaneously with $1 \times 10^{5}$ B16F0-hCD19 or $2.5 \times 10^{5}$ B16F10-OVA cells in PBS. When tumors were palpable, tumor measurements were recorded with a caliper three to four times a week and the tumor size was calculated in $\mathrm{mm}^{2}$ (length $\times$ width). On day $12,1.5 \times 10^{6} \mathrm{CAR}$ T cells or $1 \times 10^{6} \mathrm{OT}-\mathrm{I} \mathrm{T}$ cells were adoptively transferred into tumor-bearing mice. On day 20 , tumors were collected from the mice and placed into C tubes (Miltenyi Biotec) containing RPMI 1640 with $10 \% \mathrm{FBS}$ and collagenase $\mathrm{D}\left(1 \mathrm{mg} \mathrm{ml}^{-1}\right.$; Roche), hyaluronidase $\left(30 \mathrm{U} \mathrm{ml}^{-1}\right.$; Sigma-Aldrich) and DNase I (100 $\mathrm{g} \mathrm{ml}^{-1}$; Sigma-Aldrich). Tumors were dissociated using the gentleMACS dissociator (Miltenyi Biotec), incubated with shaking at 2,000 r.p.m. for $60 \mathrm{~min}$ at $37^{\circ} \mathrm{C}$, filtered through a $70-\mu \mathrm{m}$ filter and spun down. Lymphocytes were separated using lymphocyte separation medium (MP Biomedicals; 0850494).

NFAT-AP-1 reporter assays. Primary mouse $\mathrm{CD}^{+} \mathrm{T}$ cells were isolated from the spleens of C57BL/6J mice (The Jackson Laboratory; 000664) by negative selection (EasySep; 19853). Up to $5 \times 10^{6}$ freshly isolated CD8 ${ }^{+}$cells were activated with plate-bound anti-CD3 (145-2C11) and anti-CD28 (37.51) at a final $1 \mu \mathrm{g} \mathrm{ml}^{-1}$ in $\mathrm{T}$ cell media in a six-well plate. After $24 \mathrm{~h}$, cells were transduced with retroviral supernatant at $32^{\circ} \mathrm{C}$ for $2 \mathrm{~h}$ at $2,000 \mathrm{~g}$ with $8 \mu \mathrm{g} \mathrm{ml} \mathrm{m}^{-1}$ polybrene. After transduction, cells were cultured in $\mathrm{T}$ cell media containing $100 \mathrm{U} \mathrm{ml}^{-1} \mathrm{IL}-2$. On day 2 , the same transduction was performed. On day 3 , cells were surface stained for live $\mathrm{CD}^{+}$Thyl. $1^{+}$cells as a measure of reporter activity.

Flow cytometry analysis. BD Fortessa, BD LSR III or BD Celesta flow cytometers were used for cell analysis. Cells were resuspended in FACS buffer (PBS, 1\% FBS 
and $2.5 \mathrm{mM}$ ethylenediaminetetraacetic acid (EDTA)) and filtered using a $70-\mu \mathrm{m}$ filter before running the flow cytometer. Fluorochrome-conjugated antibodies were purchased from BD Biosciences, Thermo Fisher Scientific, Miltenyi Biotec and BioLegend. For surface staining, cells were stained with $~ 1: 100-1: 200$ dilution of antibodies in FACS buffer (PBS + 1\% FBS and $2.5 \mathrm{mM}$ EDTA) for $15 \mathrm{~min}$ with Fc block (BioLegend). For cytokine staining, cells were activated with $10 \mathrm{nM}$ phorbol 12-myristate 13 -acetate, $500 \mathrm{nM}$ ionomycin and $1 \mu \mathrm{g} \mathrm{ml}^{-1}$ Golgi plug and/or Golgi Stop in T Cell Media at $37^{\circ} \mathrm{C}$ in a $10 \% \mathrm{CO}_{2}$ incubator for $4 \mathrm{~h}$. After stimulation, cells were stained for surface markers and resuspended with Fix/ Perm (BD Biosciences) buffer for $20 \mathrm{~min}$, washed with FACS buffer twice and stained for cytokines at a final concentration of 1:200 in 1× BD Perm/Wash buffer. For the detection of transcription factors, cells were stained for surface markers first, after which the Foxp3/transcriptional staining kit was used according to the manufacturer's protocol. All transcription factor antibodies were used at 1:200 dilution. All flow data were analyzed with FlowJo (version 10.6.2).

Mass cytometry by time of flight (CyTOF) analysis. On day 0, 7- to 12-week-old C57BL/6J mice were injected subcutaneously with $1 \times 10^{5} \mathrm{~B} 16 \mathrm{~F} 0$-hCD19 cells. When the tumors were palpable, tumor measurements were recorded with a caliper three to four times a week and the tumor size was calculated in $\mathrm{mm}^{2}$ (length $\times$ width). On day $12,1.5 \times 10^{6} \mathrm{CAR} \mathrm{T}$ cells were adoptively transferred into tumor-bearing mice. On day 20, tumors were collected from the mice and placed into C tubes (Miltenyi Biotec) containing RPMI 1640 with 10\% FBS and collagenase $\mathrm{D}$ ( $1 \mathrm{mg} \mathrm{ml}^{-1}$; Roche), hyaluronidase ( $30 \mathrm{U} \mathrm{ml}^{-1}$; Sigma-Aldrich) and DNase I ( $100 \mu \mathrm{g} \mathrm{ml}^{-1}$; Sigma-Aldrich). The tumors were dissociated using the gentleMACS dissociator (Miltenyi Biotec), incubated with shaking at 2,000 r.p.m. for $60 \mathrm{~min}$ at $37^{\circ} \mathrm{C}$, filtered through a $70-\mu \mathrm{m}$ filter and spun down. Lymphocytes were separated using lymphocyte separation medium (MP Biomedicals; 0850494) and sorted by flow cytometry based on forward versus side scatter gating to obtain highly purified lymphocytes. After sorting, the lymphocytes were rested in T cell media for $4 \mathrm{~h}$. Cells were washed with PBS, centrifuged at $400 \mathrm{~g}$ for $5 \mathrm{~min}$ and the supernatant was discarded by aspiration. Cells were resuspended in PBS with Cell-ID Cisplatin $(5 \mu \mathrm{M})$, incubated at $\sim 22^{\circ} \mathrm{C}$ for $5 \mathrm{~min}$ and washed with MACS staining buffer ( $2 \mathrm{mM}$ EDTA and $2 \%$ FBS in PBS) using $5 \times$ the volume of the cell suspension. Cells were stained with a cocktail of antibodies to surface proteins with Fc blocking for $15 \mathrm{~min}$ at $\sim 22^{\circ} \mathrm{C}$, washed with MACS staining buffer, then fixed and permeabilized using a FoxP3 staining buffer kit (eBioscience) and stained for $1 \mathrm{~h}$ at $\sim 22^{\circ} \mathrm{C}$ with a cocktail of antibodies to intracellular proteins. Cells were washed twice with Perm/Wash buffer, fixed with $1.6 \%$ paraformaldehyde for $10 \mathrm{~min}$ at $\sim 22^{\circ} \mathrm{C}$ and washed twice again with Perm/Wash buffer. Cells were stained with Cell-ID Intercalator-Ir in Fix/Perm buffer overnight at $4{ }^{\circ} \mathrm{C}$ before analysis of the sample using a CyTOF mass spectrometer. All CyTOF data were analyzed with FlowJo (version 10.6.2) or the OMIQ.ai analysis platform.

Cell sorting. Cell sorting was performed by the LJI flow cytometry core using FACSAria I, FACSAria II or FACSAria Fusion (BD Biosciences) flow cytometers. For transcriptional profiling using Smart-seq, 10,000 cells were sorted from the Live/Dead dye-negative $\mathrm{CD}^{+}$Thy $1.1^{+} \mathrm{GFP}^{+}$population of the isolated TILs or cultured $\mathrm{CD}^{+} \mathrm{T}$ cells. The cells were resuspended in FACS buffer and filtered with a 70- $\mu \mathrm{m}$ filter before sorting. For ATAC-seq, 50,000 live cells were sorted using the same procedure as for Smart-seq. Cells were sorted into $1.5 \mathrm{ml}$ microfuge tubes containing $500 \mu \mathrm{l} 50 \% \mathrm{FBS}$. The sorted cells were washed with cold PBS twice before further procedures.

Antibodies. The following antibodies were used: BUV395 rat anti-mouse CD8 $\alpha$ (clone 53-6.7; BD Biosciences; 563786); and BV711 anti-rat CD90/mouseCD90.1 (Thy1.1) (clone OX-7; BioLegend 202539).

Primary cell culture. Splenic CD8 ${ }^{+} \mathrm{T}$ cells from C57BL/6 mice were isolated using a Dynabeads Untouched Mouse CD8 Cells Kit (Invitrogen) or EasySep Mouse $\mathrm{CD}^{+} \mathrm{T}$ Cell Isolation Kit (STEMCELL Technologies) following the manufacturer's protocols, following which $3 \times 10^{6} \mathrm{CD}^{+} \mathrm{T}$ cells per well were stimulated with $1 \mu \mathrm{g} \mathrm{ml}^{-1}$ anti-CD3 and anti-CD28 in T cell media in a six-well plate for $1 \mathrm{~d}$, then removed from the plates and retrovirally transduced using $15 \mu \mathrm{g} \mathrm{ml}^{-1}$ polybrene at $37^{\circ} \mathrm{C}$ followed by centrifugation at $2,000 \mathrm{~g}$ for $1 \mathrm{~h}$. After transduction, cells were cultured in house-made $\mathrm{T}$ cell media containing $100 \mathrm{U} \mathrm{ml}^{-1}$ human IL-2. A second transduction was performed the next day using the same protocol, after which the cells were cultured in T cell media with $100 \mathrm{U} \mathrm{ml}^{-1}$ human IL-2 for $3 \mathrm{~d}$.

Human CAR T cell experiments. Human $\mathrm{CD}^{+} \mathrm{T}$ cells were stimulated with a Dynabeads Human T-Activator CD3/CD28 (Gibco) in X-Vivo (Lonza) medium. After $2 \mathrm{~d}$, Dynabeads were removed from the cells and the cells were lentivirally transduced using retronectin-coated plates $\left(20 \mu \mathrm{g} \mathrm{ml}^{-1}\right)$ at $32^{\circ} \mathrm{C}$ followed by centrifugation at $2,000 \mathrm{~g}$ for $2 \mathrm{~h}$. Cells were expanded for $2 \mathrm{~d}$ in X-Vivo medium with $500 \mathrm{U} \mathrm{ml}^{-1}$ human IL-2. Human CAR T cells were enriched by positive selection for NGFR using MACS columns and beads (Miltenyi Biotec). cytotoxicity was calculated as $\left.1-\left(R_{5} / R_{0}\right)\right) \times 100$, where $R_{5}=$ (target cells (percentage of total) at $5 \mathrm{~h}) /($ effector cells (percentage of total) at $5 \mathrm{~h})$ and $R_{0}=($ target cells (percentage of total) at $0 \mathrm{~h}) /($ effector cells (percentage of total) at $0 \mathrm{~h})$.

In vitro proliferation assay. CellTrace Violet-labeled CAR $\mathrm{T}$ cells were cultured in $\mathrm{X}$-Vivo media with $500 \mathrm{U} \mathrm{ml}^{-1}$ human IL-2 for $4 \mathrm{~d}$.

ChIP-seq library preparation. pMIG- or BATF-transduced CD8 ${ }^{+} \mathrm{T}$ cells $\left(1 \times 10^{6}\right.$ cells per $\mathrm{ml}$ in culture media) were fixed with $1 \%$ formaldehyde at $\sim 22^{\circ} \mathrm{C}$ for $10 \mathrm{~min}$ with nutation. To quench the fixation, $0.5 \mathrm{ml} 2.5 \mathrm{M}$ glycine was added per $10 \mathrm{ml}$, then the cells were incubated on ice for $5 \mathrm{~min}$ and washed twice with cold PBS. Fixed cells were transferred to low-binding tubes with $1 \mathrm{ml}$ cold PBS and spun down at 2,000 r.p.m. at $4^{\circ} \mathrm{C}$ for $10 \mathrm{~min}$. Cells were pelleted, snap frozen with liquid nitrogen and stored at $-80^{\circ} \mathrm{C}$ until further processing. To isolate nuclei, cell pellets were thawed on ice and the pellets were resuspended in $1 \mathrm{ml}$ Bioruptor lysis buffer (50 mM HEPES (pH 7.5), $150 \mathrm{mM} \mathrm{NaCl}, 1 \mathrm{mM}$ EDTA, 10\% glycerol, $0.5 \%$ NP-40 and $0.25 \%$ Triton X-100) and incubated for $10 \mathrm{~min}$ at $4{ }^{\circ} \mathrm{C}$ with nutation. After centrifugation at $1,700 \mathrm{~g}$ and $4^{\circ} \mathrm{C}$ for $5 \mathrm{~min}$, the resulting nuclear pellets were washed twice with washing buffer (10 mM Tris- $\mathrm{HCl}$ (pH 8.0), $200 \mathrm{mM}$ $\mathrm{NaCl}, 1 \mathrm{mM}$ EDTA and $0.5 \mathrm{mM}$ EGTA). The pellets were resuspended in $100 \mu \mathrm{l}$ shearing buffer (10 mM Tris- $\mathrm{HCl}(\mathrm{pH} 8.0), 1 \mathrm{mM}$ EDTA and $1 \%$ sodium dodecyl sulfate (SDS)) and sonicated using a Bioruptor in $1.5 \mathrm{ml}$ bioruptor tubes (ten cycles; $30 \mathrm{~s}$ on and $30 \mathrm{~s}$ off). After sonication, the supernatants were transferred to $1.5 \mathrm{ml}$ low-binding tubes, and insoluble debris were removed by centrifugation at $20,000 \mathrm{~g}$. Pellets were resuspended in $100 \mu \mathrm{l}$ shearing buffer, then nine volumes of conversion buffer (10 mM Tris- $\mathrm{HCl}$ (pH 7.5), $255 \mathrm{mM} \mathrm{NaCl}, 1 \mathrm{mM}$ EDTA, $0.55 \mathrm{mM}$ EGTA. $0.11 \%$ sodium deoxycholate and $0.11 \%$ Triton X-100) were added. Chromatin was precleared with washed protein A and protein G Dynabeads for $1 \mathrm{~h}$, and the chromatin concentration was measured by Qubit. A total of $5 \%$ of the chromatin was saved as input and the chromatin was incubated with anti-BATF (Brookwood Biomedical) or anti-IRF4 (clone D9P5H; Cell Signaling Technology) antibodies and protein $\mathrm{A}$ and protein $\mathrm{G}$ Dynabeads overnight at $4{ }^{\circ} \mathrm{C}$ with rotation. The following day, bead-bound chromatin was washed twice with RIPA buffer (50 mM Tris- $\mathrm{HCl}$ (pH 8.0), $150 \mathrm{mM} \mathrm{NaCl,} 1$ mM EDTA, 1\% NP-40, $0.1 \%$ SDS and $0.5 \%$ sodium deoxycholate) and then with high salt buffer ( $50 \mathrm{mM}$ Tris- $\mathrm{HCl}$ (pH 8.0), $500 \mathrm{mM} \mathrm{NaCl}, 1 \mathrm{mM}$ EDTA, $1 \% \mathrm{NP}-40$ and $0.1 \% \mathrm{SDS}$ ), $\mathrm{LiCl}$ buffer (50 mM Tris-HCl (pH 8.0), $250 \mathrm{mM} \mathrm{LiCl,} 1 \mathrm{mM}$ EDTA, 1\% NP-40 and $1 \%$ sodium deoxycholate) and TE buffer (10 mM Tris- $\mathrm{HCl}(\mathrm{pH} 8.0)$ and $1 \mathrm{mM}$ EDTA). Chromatin was eluted with $100 \mu$ l elution buffer $\left(100 \mathrm{mM} \mathrm{NaHCO}_{3}, 1 \%\right.$ SDS and $1 \mathrm{mg} \mathrm{ml}^{-1} \mathrm{RNase}$ A) twice for $30 \mathrm{~min}$ at $37^{\circ} \mathrm{C}$ using a 1,000 r.p.m. shaking heat block. Some $5 \mu \mathrm{l}$ proteinase $\mathrm{K}\left(20 \mathrm{mg} \mathrm{ml}^{-1}\right.$; Ambion $)$ and $8 \mu \mathrm{l} 5 \mathrm{M} \mathrm{NaCl}$ were added to the eluted DNA and the samples were incubated at $65^{\circ} \mathrm{C}$ with shaking (1,200 r.p.m.) for de-crosslinking. DNA was purified with a Zymo ChIP DNA Clean \& Concentrator (Zymo Research). Libraries were prepared using NEBNex Ultra II Library Prep kits (NEB) following the manufacturer's instructions, then sequenced using an Illumina NovaSeq 6000 sequencer (50-bp paired-end reads).

ATAC-seq and RNA-seq library preparation. ATAC-seq libraries were prepared following the Omni-ATAC protocol with minor modification ${ }^{52}$. Some 50,000 cells were collected by sorting and washed twice with cold PBS at $600 \mathrm{~g}$ for $5 \mathrm{~min}$. Cell pellets were resuspended in $50 \mu \mathrm{l}$ ATAC lysis buffer ( $10 \mathrm{mM}$ Tris- $\mathrm{HCl}(\mathrm{pH} 7.4)$, $10 \mathrm{mM} \mathrm{NaCl}, 3 \mathrm{mM} \mathrm{MgCl}, 0.1 \% \mathrm{NP}-40,0.1 \%$ Tween 20 and $0.01 \%$ digitonin) and incubated on ice for $3 \mathrm{~min}$, after which $1 \mathrm{ml}$ washing buffer $(10 \mathrm{mM}$ Tris- $\mathrm{HCl}$ ( $\mathrm{pH} 7.4$ ), $10 \mathrm{mM} \mathrm{NaCl}, 3 \mathrm{mM} \mathrm{MgCl}_{2}$ and $0.1 \%$ Tween 20) was added and the cells were spun down at $1,000 \mathrm{~g}$ for $10 \mathrm{~min}$ at $4^{\circ} \mathrm{C}$. The supernatant was removed carefully, and the nuclei were resuspended in $50 \mu \mathrm{l}$ transposition mix $(25 \mu \mathrm{l} \mathrm{TD}$ buffer ( $20 \mathrm{mM}$ Tris- $\mathrm{HCl}$ (pH 7.6), $10 \mathrm{mM} \mathrm{MgCl}_{2}$ and $20 \%$ dimethylformamide), $2.5 \mu \mathrm{l} 2 \mu \mathrm{M}$ transposase, $16.5 \mu \mathrm{l} \mathrm{PBS}, 0.5 \mu \mathrm{l} 1 \%$ digitonin, $0.5 \mu \mathrm{l} 10 \%$ Tween 20 and $5 \mu \mathrm{l}$ water) and incubated at $37^{\circ} \mathrm{C}$ for $30 \mathrm{~min}$. DNA was purified with a Qiagen MinElute Kit (Qiagen). Libraries were amplified with KAPA HiFi HotStart Real-Time PCR master mix, and sequenced on an Illumina NovaSeq 6000 sequencer (50-bp paired-end reads). RNA-seq libraries were prepared following the Smart-Seq2 protocol $^{53}$ modification. Total RNA was extracted from 10,000 sorted cells using the RNeasy Plus Micro kit (Qiagen) and following the Smart-seq2 protocol as described. Libraries were prepared using the Nextera XT Library Prep kit (Illumina) and sequenced on an Illumina NovaSeq 6000 sequencer (50-bp paired-end reads).

ATAC-seq analysis. Genome browser tracks. Paired raw reads were aligned to the Mus musculus genome (mm10) using Bowtie (version 1.0.0 and -X $2000-\mathrm{m}$ 1 --best --strata -tryhard -S --fr $)^{54}$. Unmapped reads were trimmed to remove adapter sequences and clipped by one base pair (bp) with Trim_galore (version 0.4.3 $)^{55,56}$ before being aligned again (-X 2500 -m 1 --best --strata -tryhard -S --fr -v 3 -e 100). Sorted alignments from the first and second alignments were merged together with SAMtools (version 1.8) s7 $^{57}$ followed by removal of reads aligned to the mitochondrial genome using a custom perl script (version 5.18.1). Duplicated reads were removed with Picard tools' MarkDuplicates (version 1.94) ${ }^{58}$. Reads aligning to the blacklisted regions (generated by A. Boyle and A. Kundaje as part of the ENCODE and modENCODE projects ${ }^{59}$ were removed using bedtools intersect 
(version 2.27.1 $)^{60}$. Subnucleosomal fragments were defined as mapped paired reads with an insertion distance smaller than $100 \mathrm{bp}$, obtained from merged mapping results. The Tn 5 footprint was obtained by adapting J. Li's preShift.pl script to take the strand orientation of a given read to take $9 \mathrm{bp}$ around the start or end of the forward and reverse reads $([-4,5]$ and $[-5,4]$ respectively). The preShift.pl script is available at https://github.com/riverlee/ATAC/blob/master/ code/preShift.pl and the adaptation can be found at https://github.com/Edahi/ NGSDataAnalysis/blob/master/ATAC-Seq/Tn5 bed9bp full.pl. For quality control purposes, we used X. Chen's Fragment_length_density_plot.py Python script. The script is available at https:/github.com/Edahi/NGSDataAnalysis/ blob/master/ATAC-Seq/Fragment_length_density_plot.py. This program plots the histogram of the distances among the mapped usable reads. Final mapping results were processed using HOMER's makeTagDirectory program followed by the makeMultiWigHub.pl program (version 4.10 .4$)^{61}$ to produce normalized bigWig genome browser tracks for the whole mapping results, Tn5 footprint and subnucleosomal reads separately.

DARs. We used the complete fragments for peak calling using the MACS2 callpeak function (version 2.1.1.20160309 and -q 0.0001 --keep-dup all --nomodel --call-summits ${ }^{62}$. The narrowpeak files from all samples and replicates for in vivo (or in vitro) experiments were merged with bedtools merge (version 2.27.1) ${ }^{60}$ to generate a universe of peaks, which was used to obtain the $\operatorname{Tn} 5$ footprint signal from each sample. After limma-voom normalization ${ }^{63}$, performed on the Tn5 signal, and a linear model fitted to each region, computation of significance statistics for differential enrichment (accessibility) was done by empirical Bayes moderation of the standard errors, with $[-1,1]$ (lfc) as the interval for the null hypothesis. A region was considered differentially accessible if it had a $\log _{2}$ [fold change] $\geq 1$ and an adjusted $P$ value $\left(P_{\text {adj }}\right) \leq 0.05$. The Tn 5 signals from the in vivo and in vitro experiments were analyzed independent of one another. The MA plots used the merged signal from replicates. The $\mathrm{R}^{64}$ packages used included: IRdisplay $^{65}$, limma ${ }^{66}$, edgeR ${ }^{67}$, Glimma ${ }^{68}$, Mus.musculus ${ }^{69}$, RColorBrewer ${ }^{70}$, ggplot2 (ref. ${ }^{71}$ ), GenomicRanges and GenomicAlignments ${ }^{72}$ and pheatmap ${ }^{73}$.

Venn diagrams. DARs from TILs were intersected with bedtools intersect (version 2.27.1) ${ }^{60}$ with default parameters (a 1-bp overlap was considered an overlap) against the exhaustion- or activation-related regions from Mognol et al. ${ }^{36}$ (GSE88987). The overlaps were used to plot the Venn diagrams for both BATF and pMIG TILs. A one-tailed Fisher test (Fisher's exact test on $2 \times 2$ contingency tables in MATLAB $)^{74}$ was used to calculate the significance of the overlaps.

Heatmaps. The $z$ score from the limma-voom ${ }^{63}$ normalized signal from TIL and $\mathrm{CD}^{+} \mathrm{T}$ cell samples in the regions of interest (pMIG or BATF DARs from either TILs or CD8 ${ }^{+} \mathrm{T}$ cells) was clustered by the region's signal (cluster_rows $=\mathrm{T}$ ) and plotted using the R library pheatmap ${ }^{73}$.

Quartile boxplots from ChIP regions. The raw Tn5 signal ${ }^{72}$ from the 2,504 ChIP-seq regions meeting the criterion $\log _{2}[\mathrm{Tn} 5$ signal in BATF-overexpressing cells/Tn5 signal in pMIG control cells] $\geq 3$ was reads per million (RPM) normalized for both BATF and pMIG CD8 ${ }^{+} \mathrm{T}$ cells, with the RPM per replicate averaged. The regions were subdivided in quartiles with respect to the pMIG Tn5 RPM signal and the signals for both ATAC-seq and ChIP-seq data were then plotted ${ }^{71}$ together.

Known motifs analysis. A region was defined as differentially accessible when it had a twofold difference and a $P_{\text {adj }}$ (false discovery rate (FDR)) of $<0.05$. The DARs per condition and per experiment (BATF and pMIG, in vivo and in vitro) were used as input for HOMER's findMotifsGenome.pl (version 4.10.4) ${ }^{57}$.

RNA-seq analysis. Genome browser tracks. Paired reads were mapped to STAR ${ }^{75}$ using the parameters --outFilterMultimapNmax 30 --outReadsUnmapped Fastx --outSAMattributes All --outSAMprimaryFlag OneBestScore --outSAMstrandField intronMotif --outSAMtype BAM SortedByCoordinate --quantMode GeneCounts. Mapping results were processed using HOMER's makeTagDirectory ${ }^{61}$ twice: once for the individual replicates and a subsequent time merging them (for a less crowded genome browser session), followed by makeMultiWigHub.pl programs (version 4.10.4) to produce normalized bigWig genome browser tracks.

MA plots of differential gene expression (TILs). Counts per gene were obtained from STAR's STAR_gene_counts (version subread-2.0.0-source) ${ }^{75}$ Differential gene expression was analyzed with $\mathrm{R}$ (version 3.5.2) and the following packages: IRdisplay ${ }^{65}$, limma ${ }^{66}$, edgeR ${ }^{67}$, Glimma ${ }^{68}$, Mus.musculus ${ }^{69}$, RColorBrewer ${ }^{70}$ and gplots $^{76}$. In brief, count reads from STAR were read and voom normalized after both counts per million (CPM) conversion and removal of genes whose CPM was lower than 1 across less than one-third of total samples. After limma-voom normalization, performed on the gene's signal, and a linear model fitted to each gene, computation of significance statistics for differential gene expression was done by empirical Bayes moderation of the standard errors, without intervals for the null hypothesis $([0,0] \mathrm{lfc})$. A gene was considered differentially expressed if $P_{\text {adj }}$ (FDR) was $\leq 0.1$. Colors in the MA plots for these genes indicate these parameters (red indicates genes more expressed in BATF-transduced compared with control
pMIG-transduced cells, whereas blue indicates the opposite and gray indicates genes that were not differentially expressed).

MA plots of differential gene expression (in vitro). The processing was similar to that for TILs, but $[-1.2,1.2]$ (lfc) was used as the interval for the null hypothesis. A gene was considered differentially expressed if the absolute $\log _{2}$ [fold change] was $\geq 2$ and the $P_{\text {adj }}(\mathrm{FDR})$ was $\leq 0.05$.

Gene signal heatmaps. The heatmaps are composed of the top 100 most significant $\left(P_{\text {adj }}\right)$ differentially expressed genes in pMIG control cells between 0 and $6 \mathrm{~h}$ after restimulation. The limma-voom-normalized signal for all of the pMIG-, BATF- and HKE-transduced samples was $z$ score transformed gene-wise. The $z$ score-normalized data were then used to plot the heatmaps with the heatmap.2 function from the gplots ${ }^{76} \mathrm{R}$ package.

ChIP-seq analysis. Genome browser tracks. Paired raw reads were aligned to the $M$ musculus genome (version mm10) using bwa $\mathrm{b}^{77}$ mem (version 0.7.15-r1144- dirty). Unmapped reads were trimmed to remove adapter sequences and clipped by $1 \mathrm{bp}$ with Trim_galore (version 0.4 .3$)^{55,56}$ before being aligned again. Sorted alignments from the first and second alignments were merged together with SAMtools (version 1.8), followed by removal of reads aligned to the mitochondrial genome using a custom perl script (version 5.18.1). Duplicated reads were removed with Picard tools' MarkDuplicates (version 1.94) ${ }^{58}$. Reads aligning to the blacklisted regions (generated by A. Boyle and A. Kundaje as part of the ENCODE and modENCODE projects) were removed using bedtools ${ }^{60}$ intersect (version 2.27.1). Final mapping results were processed using the $\mathrm{HOMER}^{61}$ makeTagDirectory program followed by the makeMultiWigHub.pl program (version 4.10.4) to produce normalized bigWig genome browser tracks.

Venn diagram. For each sample, peaks were called using the MACS2 (version 2.1.1.20160309) ${ }^{62}$ callpeak function, using the sample's respective input dataset, a $q$ value of 0.05 and the --keep-dup all and --nomodel parameters. The narrowpeak files among replicates were merged using bedtools merge ${ }^{60}$ (version 2.27.1). To identify overlapping genes by the merged narrowpeak files per condition, we used the University of California, Santa Cruz M. musculus mm10 annotation genes. Called peaks were assigned to a gene if they overlapped with a window containing the body of the gene (the longest transcription unit for the gene locus definition) plus the 20-kilobase $(\mathrm{kb})$ region upstream of the transcription start site (TSS) and the 5-kb region downstream of the $3^{\prime}$ end of the gene. Each gene was considered only once and the whole gene set was used to find shared genes among the samples being compared. The overlap was conducted with the bedtools ${ }^{60}$ intersect function (version 2.27.1). Venn diagrams of shared overlapping genes were produced using $\mathrm{R}$ (version 3.5.2), as well as the libraries VennDiagram $^{78,79}$ and viridis ${ }^{80}$.

Probability per base pair of the BATF binding site. Peaks from BATF-transduced $\mathrm{CD}^{+} \mathrm{T}$ cells subjected to ChIP-seq with anti-BATF antibodies were functionally annotated to $\mathrm{mm} 10$ using the HOMER ${ }^{61}$ annotatePeak.pl program. The distance to the nearest TSS and gene name were filtered from the annotation results. A sublist of the genes differentially expressed between BATF- and pMIG-transduced CD8 ${ }^{+}$ T cells, identified by RNA-seq analysis, was used to subset separately the peak annotation results for genes up- and downregulated in BATF-transduced cells. The genomic histograms were generated using $\mathrm{R}(3.5 .2)^{64}$ and ggplot2 (ref. ${ }^{71}$ ) with all of the peak results, whereas the up- and downregulated histograms used the subset of genes generated above. The percentage of genes closer than $20 \mathrm{~kb}$ was obtaining by taking the absolute value to the closest TSS that was $\leq 20 \mathrm{~kb}$. The distances were numerically sorted and an empirical cumulative distribution function was generated based on the data.

Removal of spurious peaks. All of the peaks from all of the different conditions and replicates were merged into a singularity table, keeping track of which condition belonged to which region. For the superset of peaks belonging to the $\alpha$ BATF immunoprecipitation, we kept the peaks whose average RPM signal across the pMIG-, BATF- and HKE-transduced input samples was lower than 0.75 times the $\alpha$ BATF immunoprecipitation RPM signal from BATF-overexpressing cells. Similarly, for the aIRF4 immunoprecipitation superset, we kept peaks where said input signal was lower than 0.75 times that of the $\alpha$ IRF4 immunoprecipitation RPM signal from pMIG control cells. These filtered supersets were used for all subsequent analyses.

Normalized $\alpha I R F 4$ ChIP-seq reads report accurately on IRF4 binding. It cannot be taken for granted that a difference in the normalized $\alpha$ IRF4 signal (in RPM) between pMIG and BATF-overexpressing cells reports on a change in IRF4 binding at the peak in question. The general issue is that normalization of the IRF4 signal at a particular peak to total mapped reads introduces a second independent variable into the measurement. If, for example, there was free IRF4 in the nucleus of pMIG cells, and if overexpressed BATF recruited this additional IRF4 to sites in DNA, then a greater total amount of IRF4-bound DNA would be precipitated from BATF-overexpressing cells. For any individual site where exactly the same amount 
of IRF4-bound DNA was precipitated as from pMIG cells, the normalization would result in an artifactually lower RPM value.

To address this issue, we utilized a subset of nonspecific background DNA regions that were equally represented in the input samples and immunoprecipitated samples from the same cells. The reads mapping to these regions in immunoprecipitated samples-which seem to represent a low fraction of input DNA carried along by nonspecific binding to the protein A/protein G beads-can serve as an internal standard. Specifically, we selected the 20 spurious peak regions with the largest ATAC-seq signal in pMIG cells (see the preceding section), since the high total signal ensured that any fractional contribution to the signal from actual IRF4 binding would be negligible. The spurious $\alpha$ IRF4 ChIP-sec signal from these regions was consistently the same in BATF-overexpressing and pMIG cells (Extended Data Fig. 9), implying that normalization did not distort the comparison between BATF-overexpressing and pMIG samples, and that a decrease in the normalized $\alpha I R F 4$ signal for an individual specific $\alpha$ IRF4 peak meant that there was an actual decrease in IRF4 binding at that peak.

Scatter and contour plots. Each scatterplot is based on the $\log _{2}$ of the RPM immunoprecipitation signal of a subset of regions representing those of interest (for example, the $\alpha$ BATF immunoprecipitation signal from BATF-overexpressing cells versus the $\alpha \mathrm{BATF}$ immunoprecipitation signal from pMIG control cells). We took the union of peaks for the illustrated samples and fetched the $\alpha$ IRF 4 and/or $\alpha$ BATF average RPM immunoprecipitation signal (as indicated in the graphs) followed by a $\log _{2}$ transformation. These normalized signals were then processed in $\mathrm{R}$ using ggplot's function geom_bin $2 \mathrm{~d}$ (bins $=300$ ) for the scatterplots (density; that is, occurrences of points per region) and geom_density_2d(bins=30) for the contour plots ${ }^{71}$.

Overlap measurement as reads-in-peaks percentage. For the Venn diagrams of Fig. $6 \mathrm{a}$ and Extended Data Fig. 8b, we took the union of the peaks in the two conditions considered (for example, the union of $\alpha$ BATF ChIP-seq peaks from BATF-overexpressing and pMIG control cells), conserving the information on whether an individual peak was unique to one condition or shared between the two conditions. For each condition, we divided the number of reads that mapped to peaks shared by both conditions by the total reads mapped to peaks in that condition, obtaining the reads-in-peaks percentage in shared peaks and-by complementation-the reads-in-peaks percentage in unique peaks.

Histograms of signal distribution among subsets of peaks. For the histograms of Fig. $6 \mathrm{a}$ and Extended Data Fig. 8b, the identities of peaks that were unique to a specified condition or shared with a second condition were used to fetch the RPM-normalized ChIP-seq signals for peaks in each subset. The values were then $\log _{2}$ transformed, the median value was calculated from the $\log _{2}$-transformed data and its distribution was plotted as a histogram using R's ggplot 2 function geom_histogram ${ }^{71}$.

Heatmaps. We used the deepTools ${ }^{81}$ computeMatrix function (with the parameters --referencePoint center -a 1000 -b 1000 --binSize 50 --averageTypeBins mean --missingDataAsZero -p 4) to compute the signal matrices across all of the conditions. The regions that were used were the input-corrected peaks (one peakset per condition). The bigWig datasets used to fetch the signal were the HOMER-normalized bigWigs (the same ones used in the genome browser track). We then proceeded to give this program's output as input to the deepTools plotHeatmap function (with the parameters --averageType mean --plotType se --averageTypeSummaryPlot mean --sortRegions descend --sortUsing mean --sortUsingSamples 6 --refPointLabel Center --missingDataColor yellow).

Statistical analysis. No statistical method was used to predetermine sample size. No data were excluded from the analyses. Tumor-bearing mice were randomly assigned to adoptive-transfer treatment groups. In most cases, tumor sizes were measured in a blinded manner by DLAC staff, except during the holiday season or when the institute was under restricted access due to the COVID-19 shutdown. Investigators were not blinded to sample identity when analyzing $\mathrm{T}$ cells recovered from the tumors. Details of the sample sizes, replicates and statistical tests used are provided in the individual figure captions.

Reporting Summary. Further information on research design is available in the Nature Research Reporting Summary linked to this article.

\section{Data availability}

The RNA-seq, ATAC-seq and ChIP-seq data have been deposited at the Gene Expression Omnibus (GSE154747). Source data are provided with this paper. Other data that support the findings of this study are available from the corresponding authors upon request.

\section{Code availability}

The base code used to preprocess the ATAC-seq, RNA-seq and ChIP-seq data and the code used for the analysis of differential gene expression have been deposited in GitHub at https://github.com/Edahi/NGSDataAnalysis.

\section{References}

49. Nicholson, I. C. et al. Construction and characterisation of a functional CD19 specific single chain Fv fragment for immunotherapy of B lineage leukaemia and lymphoma. Mol. Immunol. 34, 1157-1165 (1997).

50. Roybal, K. T. et al. Precision tumor recognition by T cells with combinatorial antigen-sensing circuits. Cell 164, 770-779 (2016).

51. Hooijberg, E., Bakker, A. Q., Ruizendaal, J. J. \& Spits, H. NFAT-controlled expression of GFP permits visualization and isolation of antigen-stimulated primary human T cells. Blood 96, 459-466 (2000).

52. Corces, M. R. et al. An improved ATAC-seq protocol reduces background and enables interrogation of frozen tissues. Nat. Methods 14, 959-962 (2017)

53. Picelli, S. et al. Full-length RNA-seq from single cells using Smart-seq2. Nat. Protoc. 9, 171-181 (2014).

54. Langmead, B., Trapnell, C., Pop, M. \& Salzberg, S. L. Ultrafast and memory-efficient alignment of short DNA sequences to the human genome. Genome Biol. 10, R25 (2009).

55. Krueger, F. Trim galore: a wrapper tool around Cutadapt and FastQC to consistently apply quality and adapter trimming to FastQ files. (2015); https:// www.bioinformatics.babraham.ac.uk/projects/trim_galore/

56. Martin, M. Cutadapt removes adapter sequences from high-throughput sequencing reads. ENBnet J. 17, 10-12 (2011).

57. Li, H. et al. The Sequence Alignment/Map format and SAMtools. Bioinformatics 25, 2078-2079 (2009).

58. Broad Institute. Picard Toolkit (2018); http://broadinstitute.github.io/picard/

59. Amemiya, H. M., Kundaje, A. \& Boyle, A. P. The ENCODE blacklist: identification of problematic regions of the genome. Sci. Rep. 9, 9354 (2019).

60. Quinlan, A. R. \& Hall, I. M. BEDTools: a flexible suite of utilities for comparing genomic features. Bioinformatics 26, 841-842 (2010).

61. Heinz, S. et al. Simple combinations of lineage-determining transcription factors prime cis-regulatory elements required for macrophage and B cell identities. Mol. Cell 38, 576-589 (2010).

62. Zhang, Y. et al. Model-based analysis of ChIP-seq (MACS). Genome Biol. 9 R137 (2008)

63. Law, C. W., Chen, Y., Shi, W. \& Smyth, G. K. voom: precision weights unlock linear model analysis tools for RNA-seq read counts. Genome Biol. 15, R29 (2014).

64. R Development Core Team. R: A Language and Environment for Statistical Computing (R Foundation for Statistical Computing, 2018).

65. Kluyver, T., Angerer, P. \& Schulz, J. IRdisplay: 'Jupyter' Display Machinery. R package v0.7.0 (2016); https://CRAN.R-project.org/package=IRdisplay

66. Ritchie, M. E. et al. limma powers differential expression analyses for RNA-sequencing and microarray studies. Nucleic Acids Res. 43, e47 (2015).

67. Robinson, M. D., McCarthy, D. J. \& Smyth, G. K. edgeR: a Bioconductor package for differential expression analysis of digital gene expression data. Bioinformatics 26, 139-140 (2010).

68. Su, S. et al. Glimma: interactive graphics for gene expression analysis. Bioinformatics 33, btx094 (2017).

69. Bioconductor Core Team. Mus.musculus: Annotation package for the Mus. musculus object. R package v1.3.1 (2015).

70. Neuwirth, E. RColorBrewer: ColorBrewer palettes. R package v1.1-2 (2014); https://CRAN.R-project.org/package=RColorBrewer

71. Wickham, H. in ggplot2: Elegant Graphics for Data Analysis (Use R!) 147-168 (Springer, 2016)

72. Lawrence, M. et al. Software for computing and annotating genomic ranges. PLoS Comput. Biol. 9, e1003118 (2013).

73. Kolde, R. pheatmap: Pretty heatmaps. R package v1.0.12 (2019); https:// CRAN.R-project.org/package $=$ pheatmap

74. MATLAB v7.10.0 (R2010a) (The MathWorks, 2010).

75. Dobin, A. et al. STAR: ultrafast universal RNA-seq aligner. Bioinformatics 29, 15-21 (2013).

76. Warnes, G. R. et al. gplots: Various R programming tools for plotting data. R package v3.0.1.1 (2009); https://CRAN.R-project.org/package=gplots

77. Li, H. \& Durbin, R. Fast and accurate short read alignment with BurrowsWheeler transform. Bioinformatics 25, 1754-1760 (2009).

78. Chen, H. VennDiagram: Generate high-resolution Venn and Euler plots. R package v1.6.20 (2018); https://CRAN.R-project.org/package=VennDiagram

79. Chen, H. \& Boutros, P. C. VennDiagram: a package for the generation of highly-customizable Venn and Euler diagrams in R. BMC Bioinformatics 12, 35 (2011).

80. Garnier, S. Colorblind-friendly color maps for R. R package v0.6.1. (2018); https://CRAN.R-project.org/package=viridis

81. Ramírez, F., Dündar, F., Diehl, S., Grüning, B. A. \& Manke, T. deepTools: a flexible platform for exploring deep-sequencing data. Nucleic Acids Res. 42, W187-W191 (2014)

\section{Acknowledgements}

We thank C. Kim, D. Hinz and C. Dillingham of the LJI Flow Cytometry Core Facility for the CyTOF experiments; D. Hinz, C. Dillingham, S. Ellis, M. Haynes, S. Sehic and 
C. Kim for cell sorting; J. Day, C. Kim and K. Tanguay of the LJI Next Generation Sequencing Facility for sequencing; the LJI DLAC and the animal facility for excellent support; and R. Nowak for excellent laboratory management. This work was funded in part by NIH R01 grants AI109842 and AI040127 (to P.G.H. and A.R.); U01 grant DE028227 (to A.R.); NIH S10 instrumentation grants RR027366 and OD018499 (to the LJI); AACR-Genentech Immuno-oncology Research Fellowship 18-40-18-SEO and Donald J. Gogel Cancer Research Institute Irvington Fellowship (to H.S.); University of California Institute for Mexico and the United States (UC MEXUS) and El Consejo Nacional de Ciencia y Tecnología (UC MEXUS/CONACYT) predoctoral fellowship (to E.G.-A.); NIH T32 predoctoral training grant in the University of California, San Diego Cardiovascular Bioengineering Training Program (to W.Z.); Cancer Research Institute Irvington Fellowship (to C.Y.); an Independent Investigator Fund (LJ/Kyowa Kirin) and a Career Transition Award from the National Cancer Institute (K22CA241290 to C.-W.J. L.)

\section{Author contributions}

H.S. designed and performed the experiments, analyzed the data and prepared the sequencing libraries. E.G.-A. performed the computational analyses of the genome-wide sequencing data. W.Z. cloned the plasmid vectors, performed the NFAT-AP-1 reporter screening assays and assisted with the in vivo and in vitro experiments. C.-W.J.L. provided the pMIG-BATF and IRF4 vectors. P.R. and C.Y. assisted with the in vitro experiments. A.R. and P.G.H. supervised the study. H.S., E.G.-A., A.R. and P.G.H. interpreted the data and wrote the manuscript. All authors contributed to writing and providing feedback.

\section{Competing interests}

A.R. and P.G.H. were recipients of a grant from Lyell Immunopharma for a separate research project related to cancer immunotherapy. The La Jolla Institute for Immunology has filed a patent application, PCT/62/976,706, covering the production and use of engineered immune cells expressing BATF and IRF4. H.S., A.R. and P.G.H. are listed as inventors. The other authors have no competing interests.

\section{Additional information}

Extended data is available for this paper at https://doi.org/10.1038/s41590-021-00964-8.

Supplementary information The online version contains supplementary material available at https://doi.org/10.1038/s41590-021-00964-8.

Correspondence and requests for materials should be addressed to A.R. or P.G.H.

Peer review information Nature Immunology thanks Rahul Roychoudhuri, Harinder Singh and the other, anonymous, reviewer(s) for their contribution to the peer review of this work. Peer reviewer reports are available. L. A. Dempsey was the primary editor on this article and managed its editorial process and peer review in collaboration with the rest of the editorial team.

Reprints and permissions information is available at www.nature.com/reprints. 
a

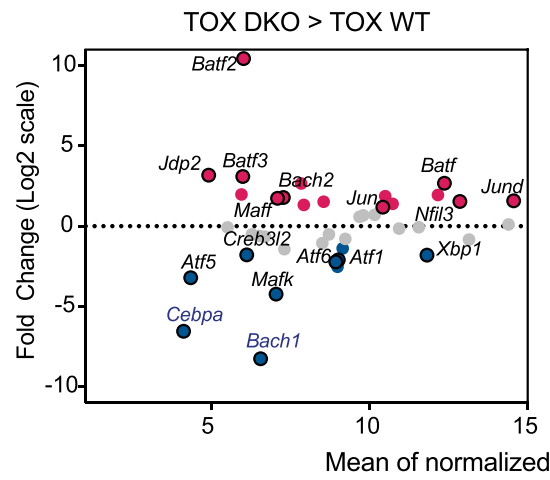

Nr4a TKO >Nr4a WT

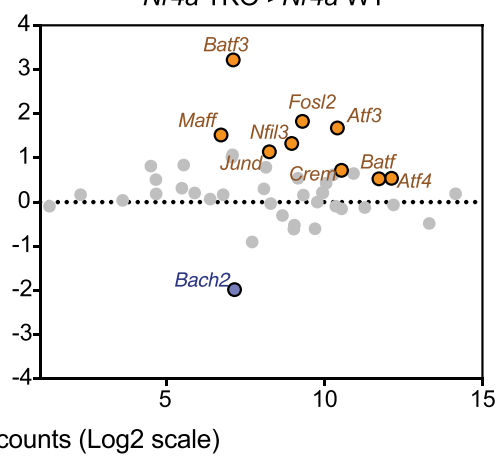

b

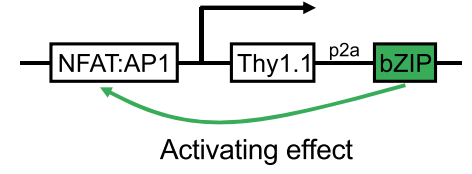

C
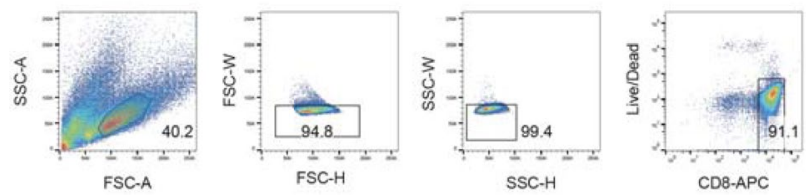

d
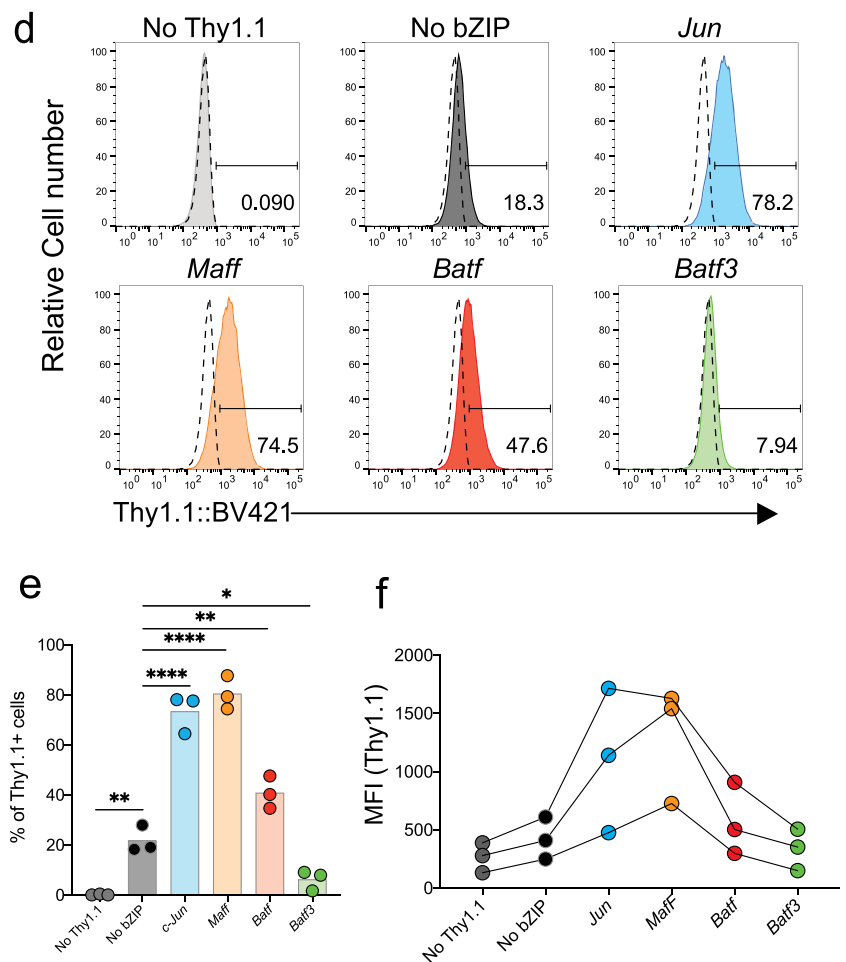

g
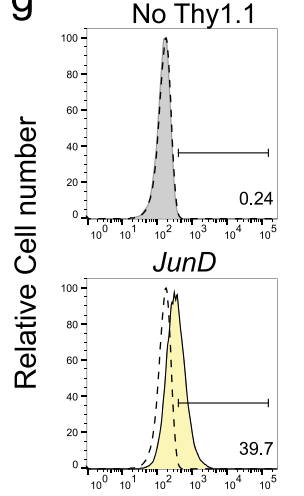

Thy 1.1::BV421

h

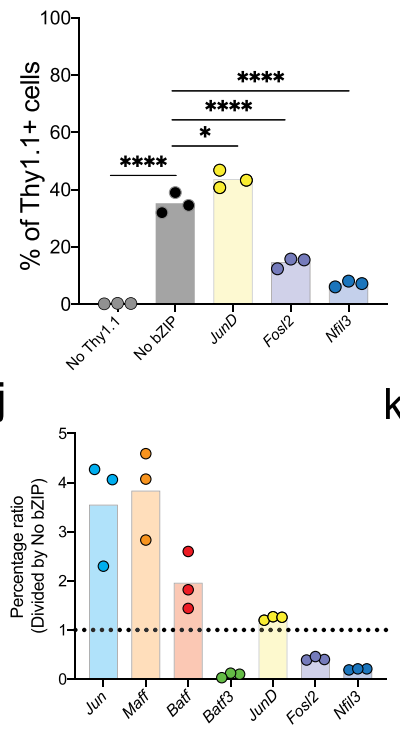

i
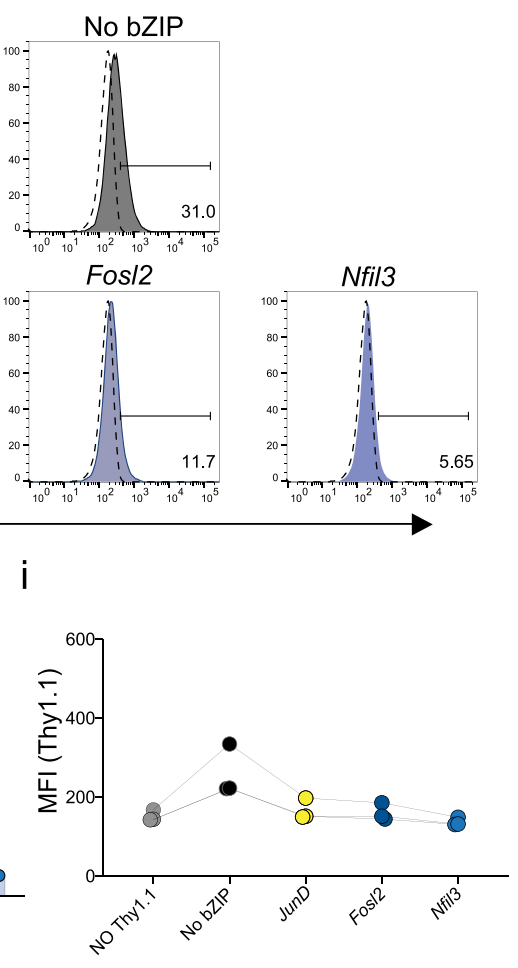

$\mathrm{k}$

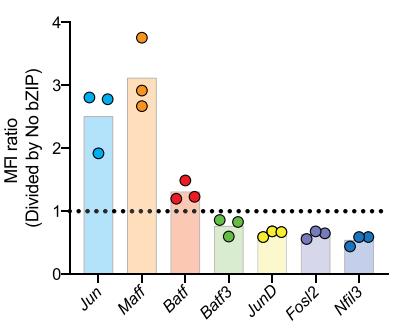

Extended Data Fig. 1 | See next page for caption. 
Extended Data Fig. 1 | Identification of bZIP transcription factors capable of increasing NFAT:AP-1 reporter activity. a, MA plots of basic region-leucine zipper (bZIP) transcription factor gene expression in TOX-depleted (TOX DKO, left) or NR4A-depleted (Nr4a TKO, right) CAR TILs5,6 - which mount increased anti-tumor responses - relative to control CAR TILs. Differentially expressed genes (adjusted $p$-value $<0.1, \log _{2}$ fold-change $\geq 0.5$ or $\leq-0.5$ ) are highlighted; selected genes are labeled. $\mathbf{b}$, Basis of the experiment to identify bZIP transcription factors that activate an NFAT:AP-1 reporter through a positive feedback loop, either directly by binding adjacent to NFAT on the NFAT:AP-1 composite site or indirectly by increasing the expression or activity of NFAT or AP-1. Mouse CD8+ T cells were transduced with retroviral expression vectors encoding a Thy1.1 reporter, separated by a P2A sequence from a co-expressed bZIP transcription factor, and under the control of six tandem NFAT:AP1 sites upstream of a minimal IL-2 promoter. Transcription factors for testing were chosen based on the data analysis in $\mathbf{a}$. c, Gating strategy for the experiments. $\mathbf{d}, \mathbf{g}$, Histograms of Thy 1.1 expression after CD8 ${ }^{+} \mathrm{T}$ cell transduction. No Thy1.1, transduced with empty retrovirus without Thy1.1 or bZIP transcription factor; No bZIP, transduced with retroviral vector encoding Thy1.1 but no bZIP transcription factor, a condition that assesses the background induction of Thy 1.1 by endogenous NFAT and AP-1; Jun, Maff, Batf, and Batf3 (d) and JunD, Fos/2, and Nfil3 (g), transduced with vector encoding the indicated bZIP transcription factor. e, h, Percentage of Thy $11^{+}$cells in three replicate experiments. $\mathbf{f}, \mathbf{i}, \mathrm{MFI}$ of Thy1.1 expression in these experiments. $\mathbf{j}, \mathbf{k}$, Results for each sample, normalized to those of the No bZIP control from the same experiment. Each circle in $\mathbf{e}, \mathbf{f}, \mathbf{h}, \mathbf{l}, \mathbf{j}$ and $\mathbf{k}$ represents one mouse. Data are representative of $(\mathbf{c}, \mathbf{d}, \mathbf{g})$ or obtained from (e, $\mathbf{f}, \mathbf{h}-\mathbf{k})$ three biological replicate experiments. Data in $\mathbf{e}$ and $\mathbf{h}$ were analyzed by one-way ANOVA. ${ }^{\star} P \leq 0.05 ;{ }^{\star \star} P \leq 0.01 ;{ }^{\star \star \star} P \leq 0.001 ;{ }^{\star \star \star \star} P \leq 0.0001$. 
a
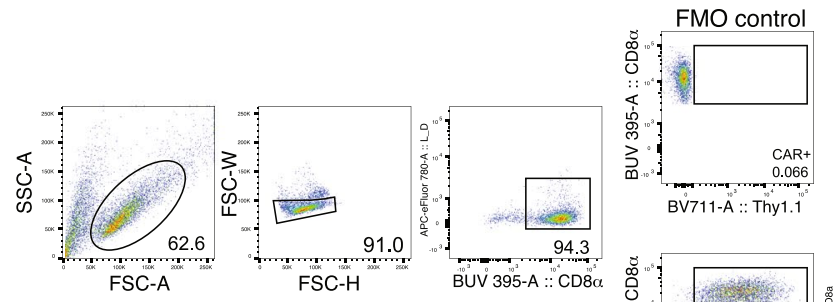

b
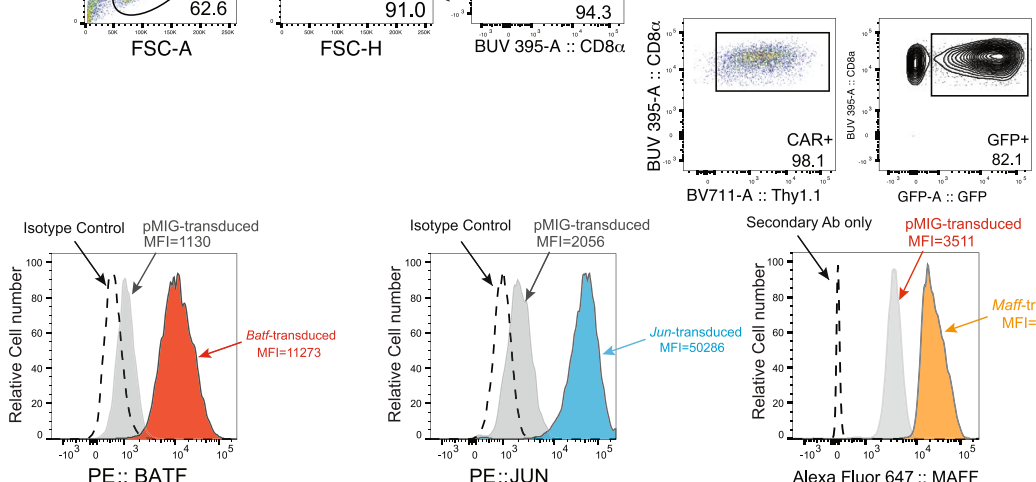

d

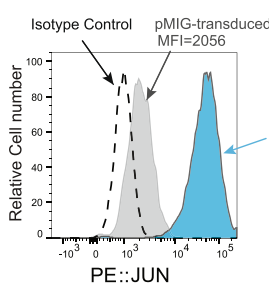

Secondary Ab only PMIG-transduced
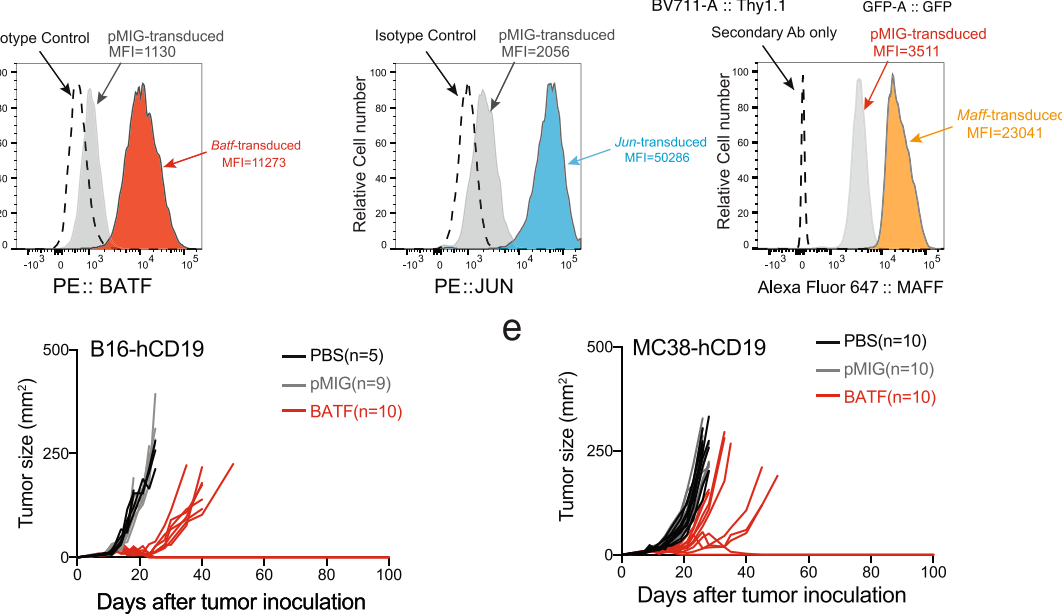

e
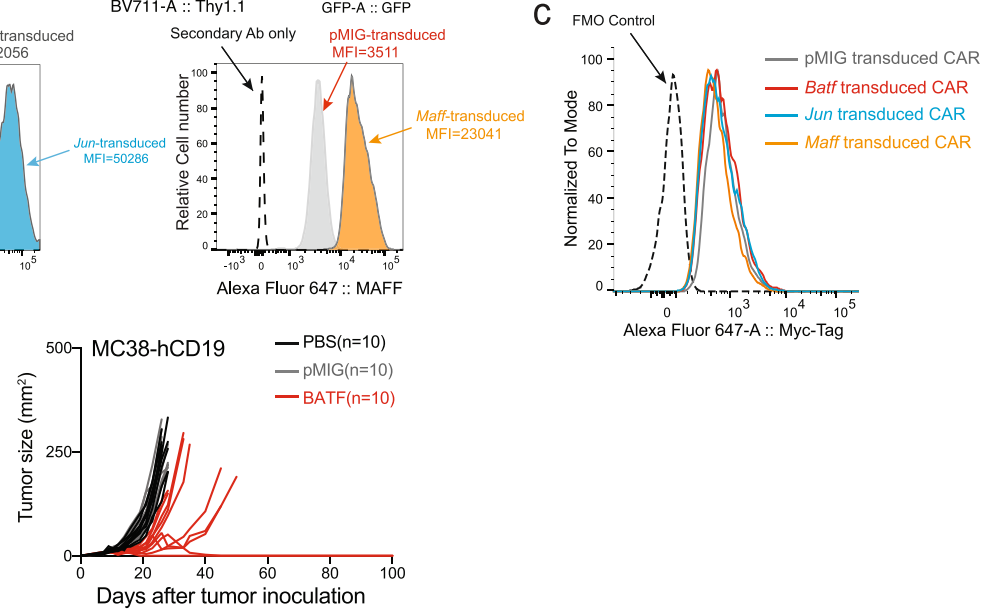

f
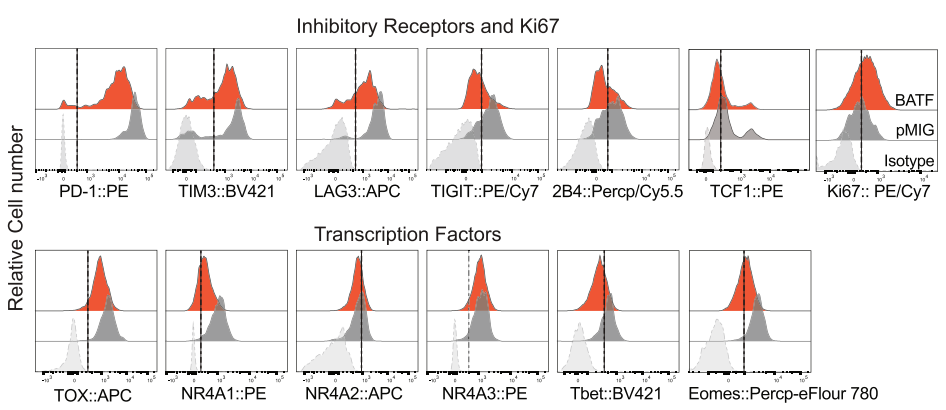

9
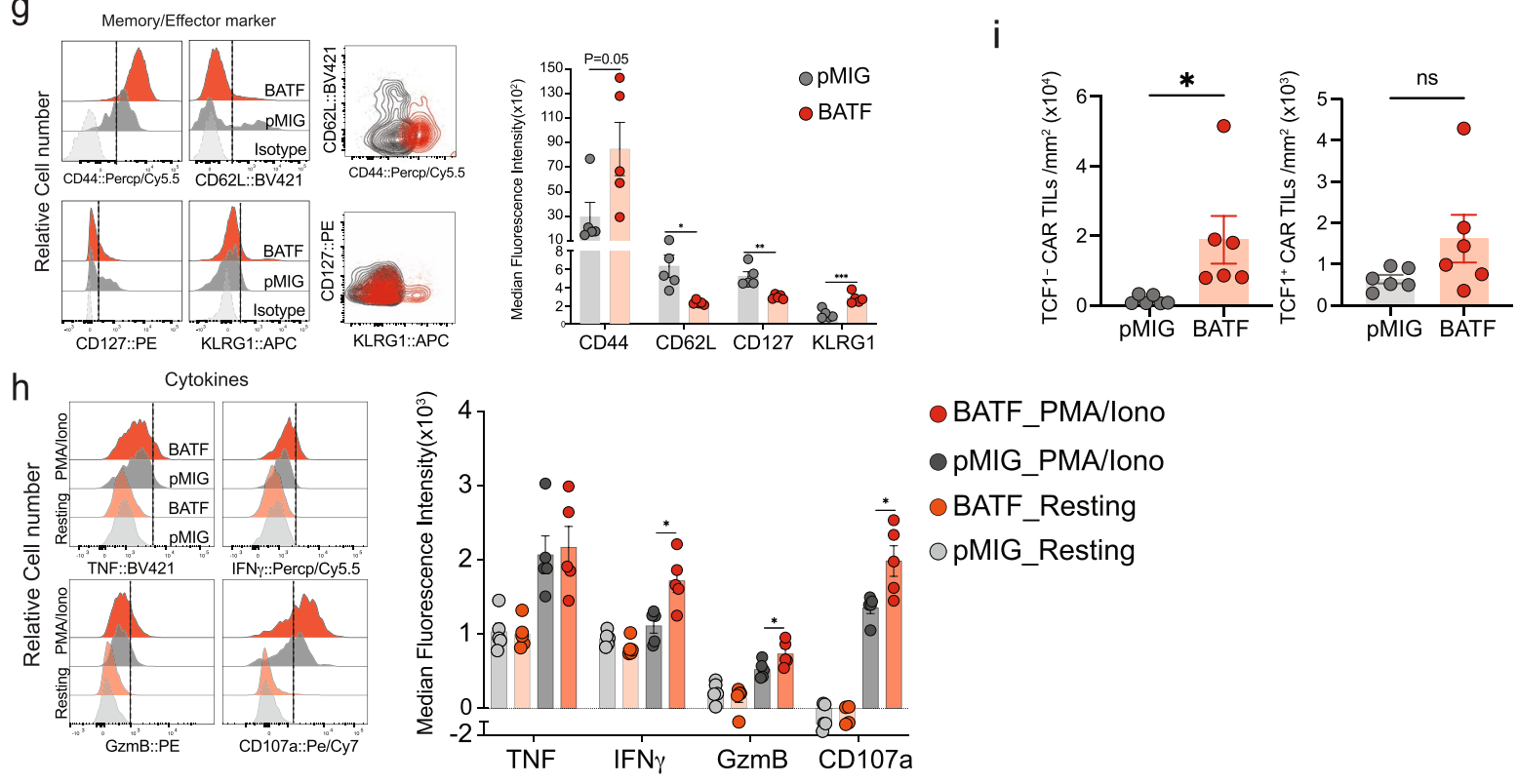

- BATF_PMA/lono

- pMIG_PMA/lono

- BATF_Resting

opMIG_Resting

Extended Data Fig. 2 | See next page for caption. 
Extended Data Fig. 2 | Phenotypic analyses of pMIG- and BATF-transduced CAR TILs. a, Retroviral transduction efficiencies for CAR and MSCV-IRESeGFP retroviral expression plasmids, assessed as expression of Thy 1.1 and GFP respectively. FMO, fluorescence-minus-one control. b, Histograms showing BATF (left), JUN (middle), and MAFF (right) expression after retroviral transduction of CD8 ${ }^{+} T$ cells with the corresponding retroviral expression plasmids or pMIG empty-vector control, assessed by flow cytometry with antibodies to the endogenous proteins. c, Histograms showing CAR expression (assessed by staining for the Myc tag) in pMIG- (grey), BATF- (red), JUN- (sky blue) and MAFF- (orange) transduced CAR T cells. d-e, Replicate tumor growth experiments using B16F0-hCD19 (d) and MC38-hCD19 (e) tumor cells. 1×105 tumor cells were injected subcutaneously into the left flank of C57BL/6 mice at day 0 (DO) in $100 \mu$ l phosphate-buffered saline (PBS); $3 \times 10^{6}$ control pMIG-, JUN-, MAFF-, or BATF-transduced CAR T cells were adoptively transferred by retro-orbital injection at day 7. Tumor sizes were measured by caliper. $\mathbf{f}$. Histograms showing expression of the indicated markers by each group of CAR TILs, assessed by flow cytometry. g, Left panels, Histograms showing expression of CD44, CD62L, CD127 and KLRG1. Middle panels, Overlaid contour plots of CD44 and CD62L (top) and CD127 and KLRG1 (bottom) in PMIG- (grey) and BATF- (red) transduced CAR TILs. Right panel, expression of the markers quantitated as MFI. h, Left panels, Histograms showing expression of TNF, IFN $\gamma$, granzyme B, and CD107a after resting in T cell media or after stimulation with PMA and ionomycin for $4 \mathrm{~h}$. Right panel, expression of the markers quantitated as MFI. i, pMIG $(n=6)$ and BATF $(n=6)$. Quantitation of $\mathrm{TCF}^{+}$and TCF1- CAR TILs. Each circle in $\mathbf{g}, \mathbf{h}$, and $\mathbf{i}$ represents one mouse, and the bar graphs represent the mean \pm standard error of mean (s.e.m.). Data in $\mathbf{d}$-i were obtained from two independent biological experiments. Data in $\mathbf{g}$, $\mathbf{h}$, and $\mathbf{i}$ were analyzed by two-tailed unpaired Student's $t$-test. 
a
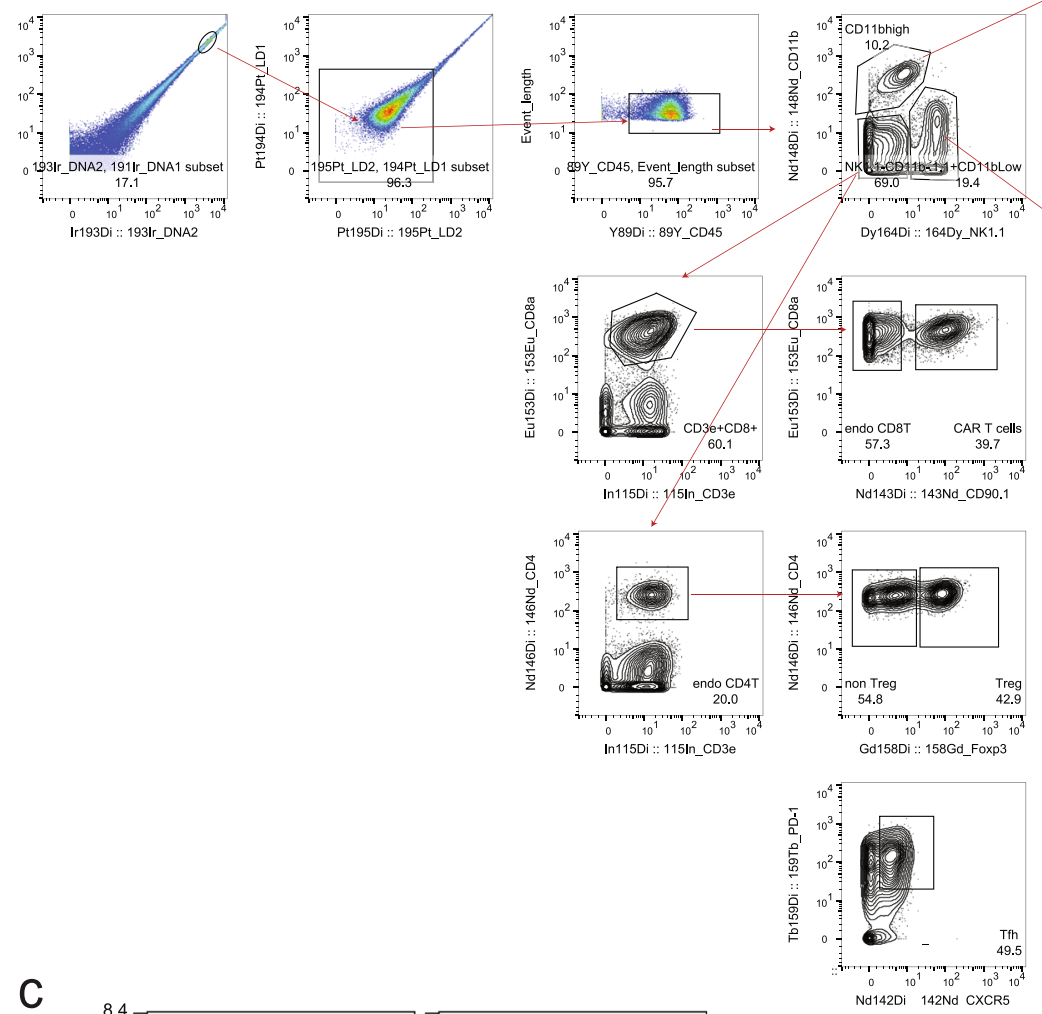

C

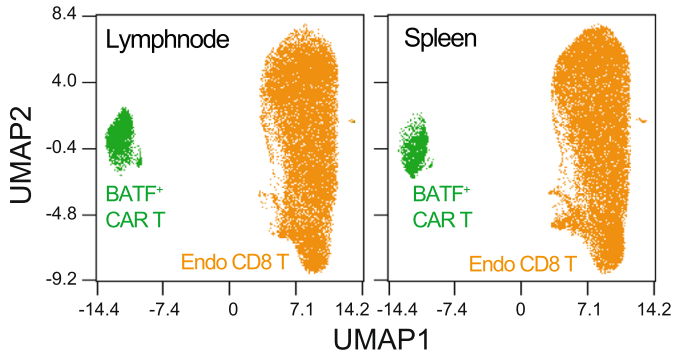

e

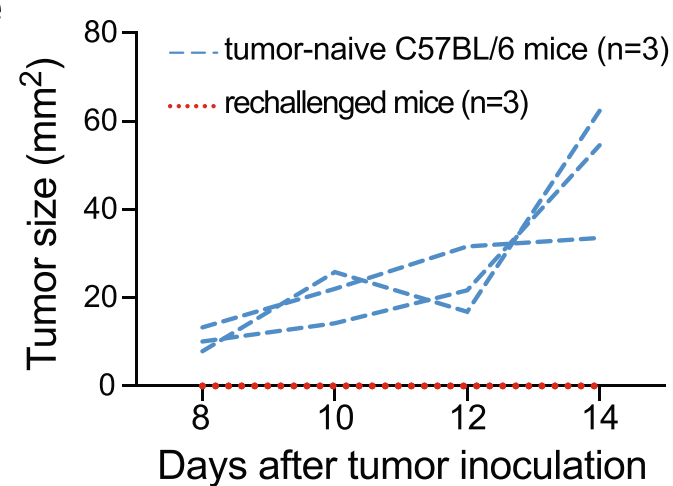

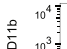

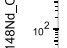

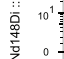

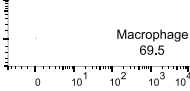

Err66Di :: 166Er_F4_80

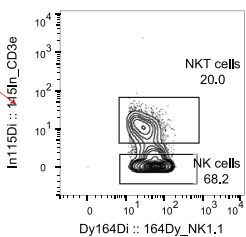

b

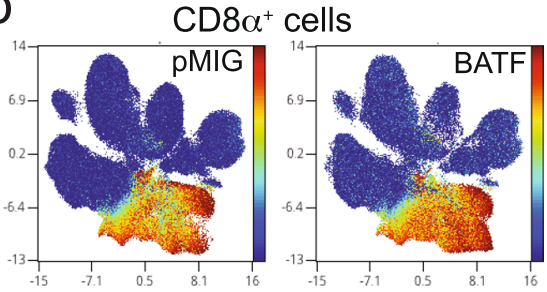

Thy1.1 (CAR TILs)
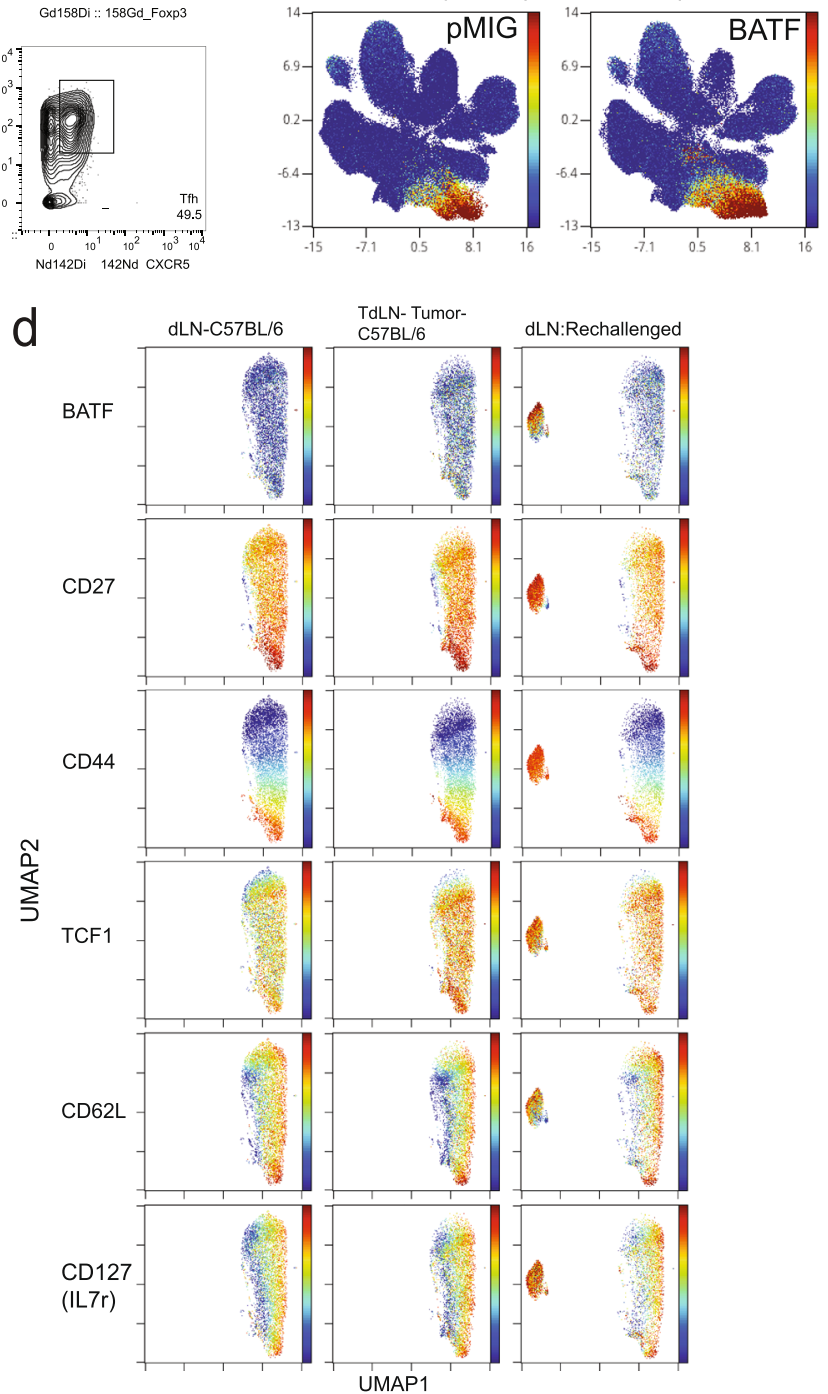

Extended Data Fig. 3 | See next page for caption. 
Extended Data Fig. 3 | Mass cytometric analyses. a, Gating strategy for mass cytometry of tumor-infiltrating lymphocytes (TILs). b, UMAP plots of data from TILs of tumour-bearing mice, showing expression of CD8 $\alpha$ (top) as a marker for all CD8 ${ }^{+} \mathrm{T}$ cells (including endogenous T cells) and Thy1.1 (bottom) as a marker for adoptively transferred pMIG- or BATF-transduced CAR T cells. c, Mass cytometric analysis of CD $8^{+} T$ cells from draining lymph nodes (left) and spleens (right) of mice in the tumor rechallenge experiments (Fig. 3). Endogenous CD8 ${ }^{+}$T cells and BATF-transduced CAR T cells are clearly distinguishable in the UMAP views. $\mathbf{d}$, Expression of selected surface markers on lymphocytes obtained from three groups of age-matched C57BL/6 mice: inguinal lymph nodes of completely unmanipulated, non-tumor-bearing mice (left panels); draining (inguinal) lymph nodes of mice inoculated with tumors 14 days previously (middle panels), and draining (inguinal) lymph nodes of rechallenged mice. The UMAP plots show that each marker is expressed by all or by a large fraction of lymph node CAR T cells of the rechallenged mice. Each group of samples in $\mathbf{c}$ and $\mathbf{d}$ was pooled from 5 mice. The data are representative of two independent biological experiments. e, Replicate of the experiment in Fig. 3e. 1×105 B16F0-hCD19 tumor cells were injected subcutaneously into the right flank of age-matched C57BL/6 mice $(n=3)$ to yield the 'tumor-naïve' control group, or into the right flank of the surviving tumor-free mice $(n=3)$ from the experiment of Extended Data Fig. 2d. In this replicate B16-hCD19 rechallenge experiment, CAR T cells accounted for $\sim 10 \%$ of CD8 ${ }^{+} \mathrm{T}$ cells in spleen and LN of the mice, and exhibited a memory phenotype (CD44 ${ }^{\text {high }}, \mathrm{CD} 62 \mathrm{~L}^{\text {high }}$, TCF ${ }^{\text {high }}$ ) as in the first experiment. 
a

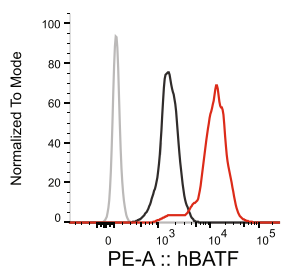

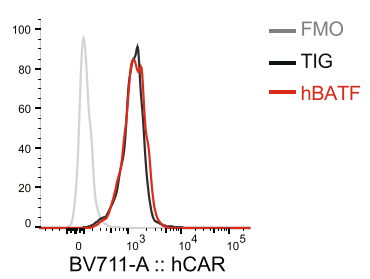

b

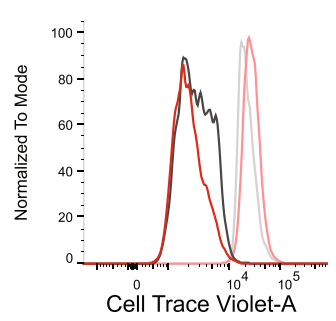

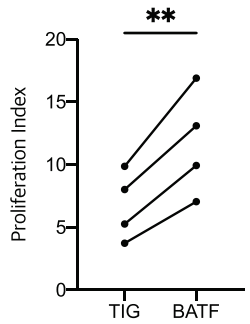

C
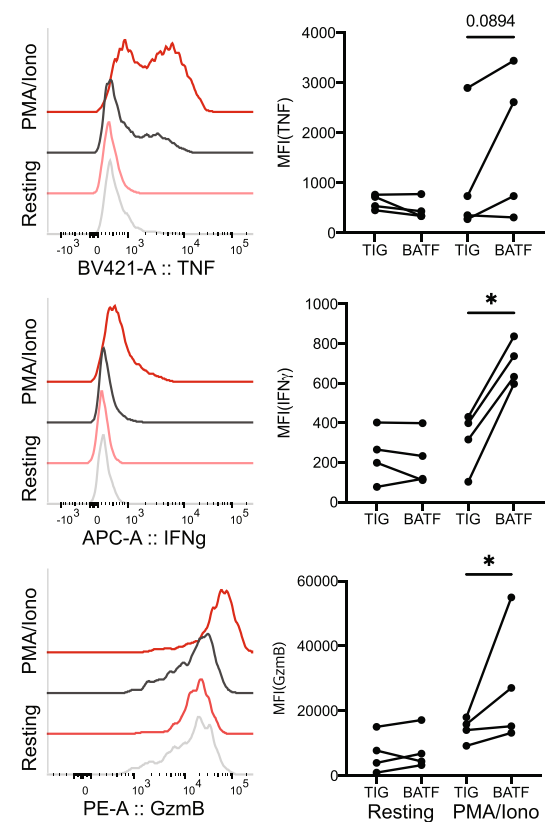

— TIG (Day 0)

- DBATF (Day 0

—TIG (Day 4)

- hBATF (Day 4$)$

d

Oh

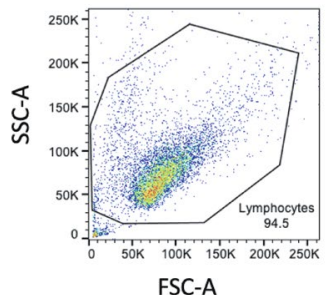

$5 h$ later

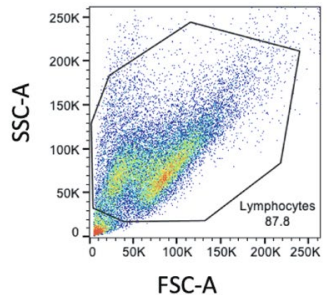

FSC-A

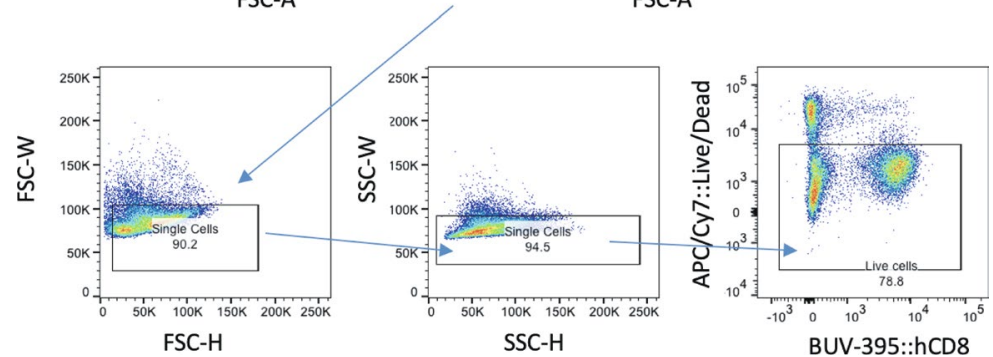

e

Oh
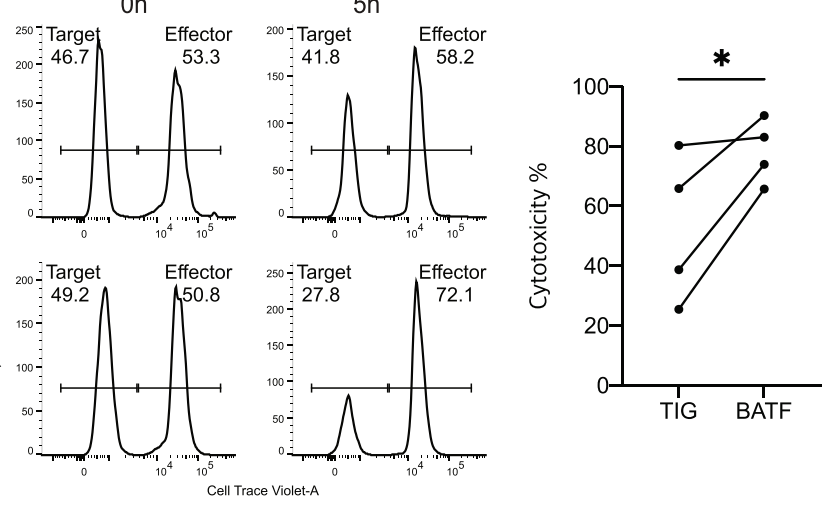

Extended Data Fig. 4 | BATF overexpression improves effector function in human CAR T cells. a, Histograms showing expression of human BATF (hBATF, left panel) and human CAR (hCAR [stained by Goat Anti-Armenian Hamster lgG $(\mathrm{H}+\mathrm{L})$ ], right panel) in the corresponding lentivirally transduced human $\mathrm{CD}^{+} \mathrm{T}$ cells. FMO, fluorescence-minus-one control; TIG, control cells transduced with the empty vector. b, Left, Histograms showing CellTrace Violet (CTV) dilution in lentivirally transduced human CD8 $8^{+}$CAR T cells. Right, Proliferation index calculated as (Day 0 CTV MFI) / (Day 4 CTV MFI). c, Histograms showing expression of the indicated cytokines after resting in X-Vivo media or after stimulation with PMA and ionomycin for $4 \mathrm{~h}$. d-e, Human CAR T cells were labeled with CellTrace Violet and co-cultured with NALM 6 cells for $5 \mathrm{~h}$. $\mathbf{d}$, Gating strategy for in vitro cytotoxicity assay of control (TIG) or hBATF-transduced human CAR T cells. e, Histograms showing ratio between target cells (NALM6) and effector cells (human CAR T cells). Percent cytotoxicity was calculated as (1- (R5/RO)) $\times 100$, where R5 $=$ (target cells as \% of total at $5 \mathrm{~h}$ ) / (effector cells as \% of total at $5 \mathrm{~h}$ ), $\mathrm{RO}=($ target cells as \% of total at $\mathrm{O} \mathrm{h}) /$ (effector cells as \% of total at $\mathrm{Oh}$ ). Each dot in b-e represents an individual donor. Data were obtained from four biological experiments and analyzed by one-tailed unpaired Student's $t$-test. 
a

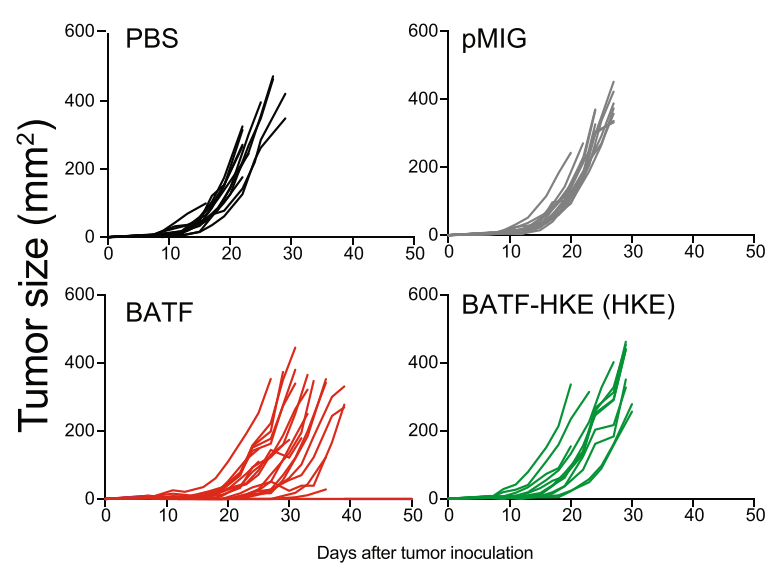

b

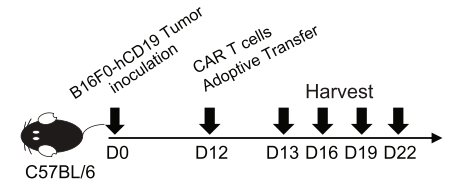

C

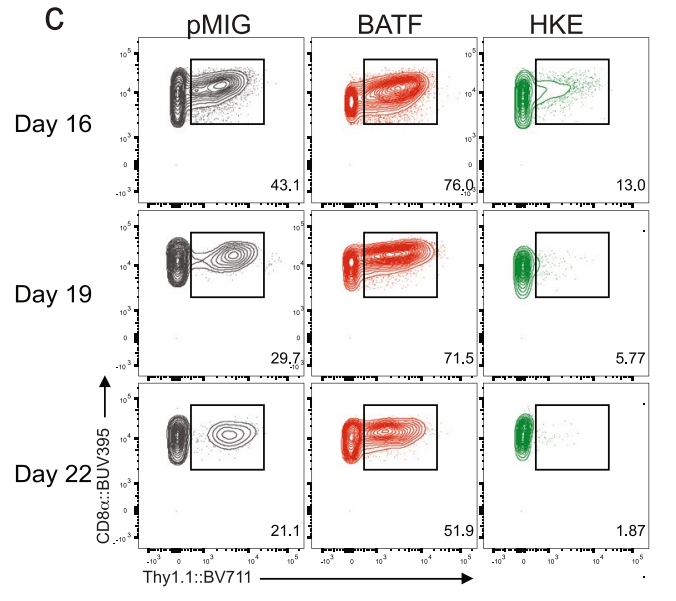

i
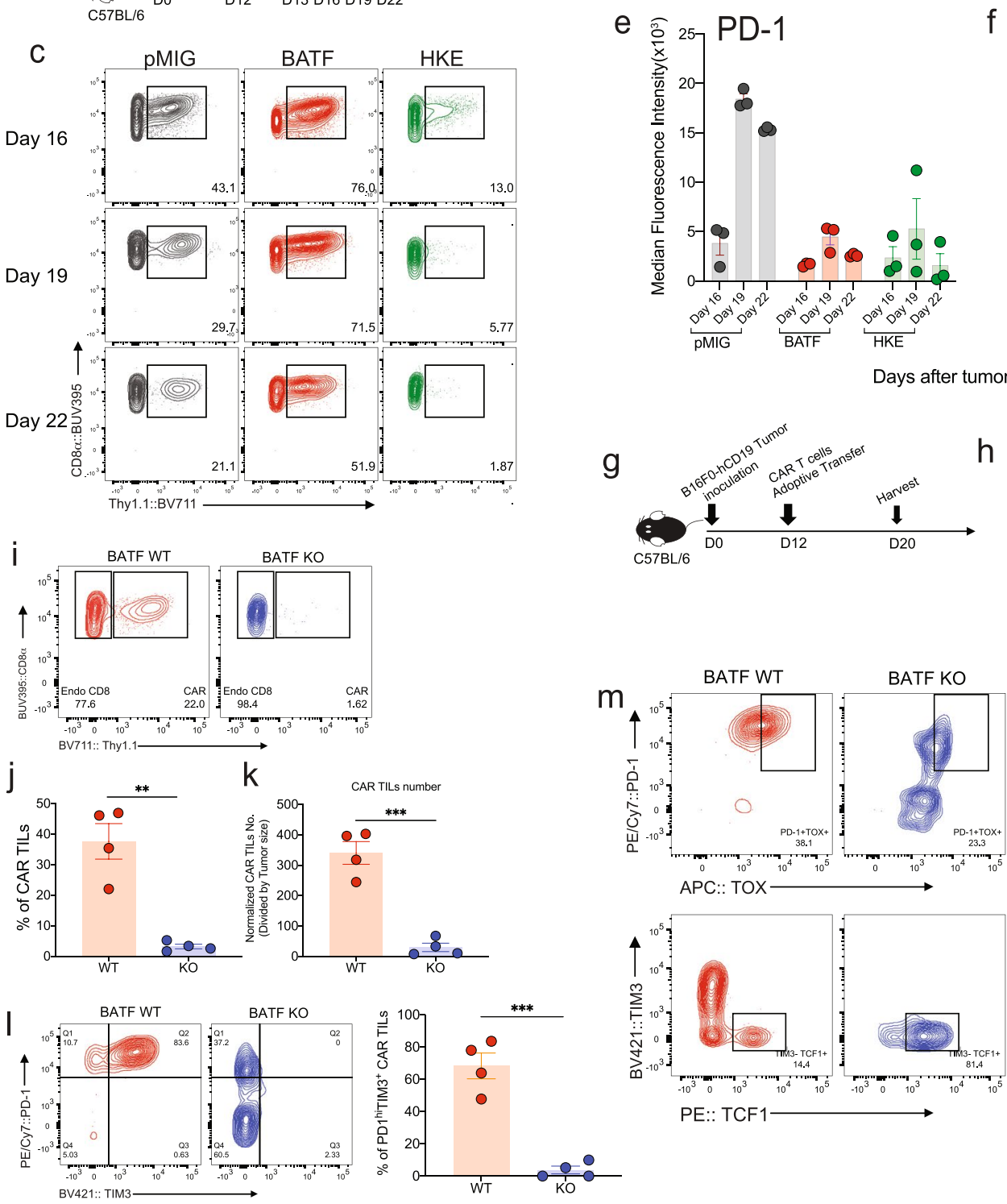

$f{ }^{\widehat{\delta}}{ }^{15} 7$ TIM3

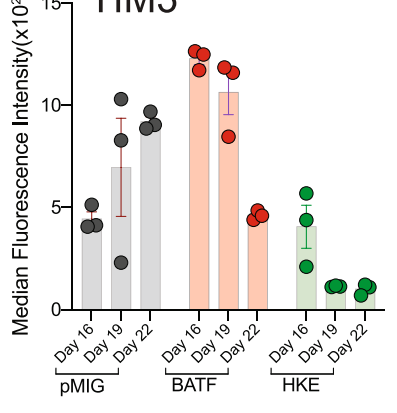

Days after tumor inoculation
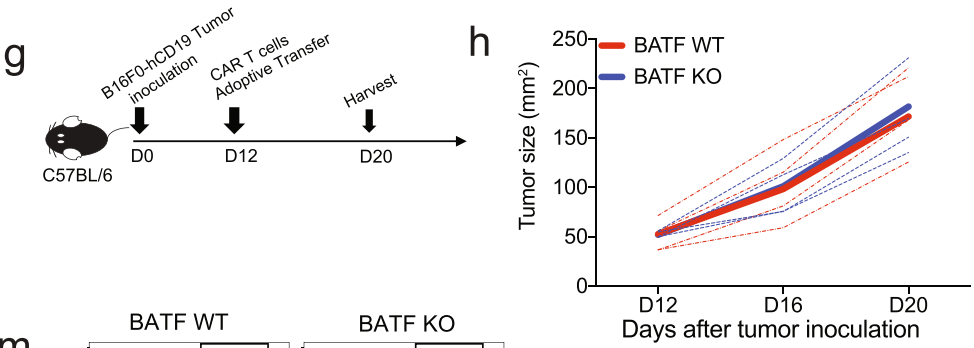

PE:: TCF1
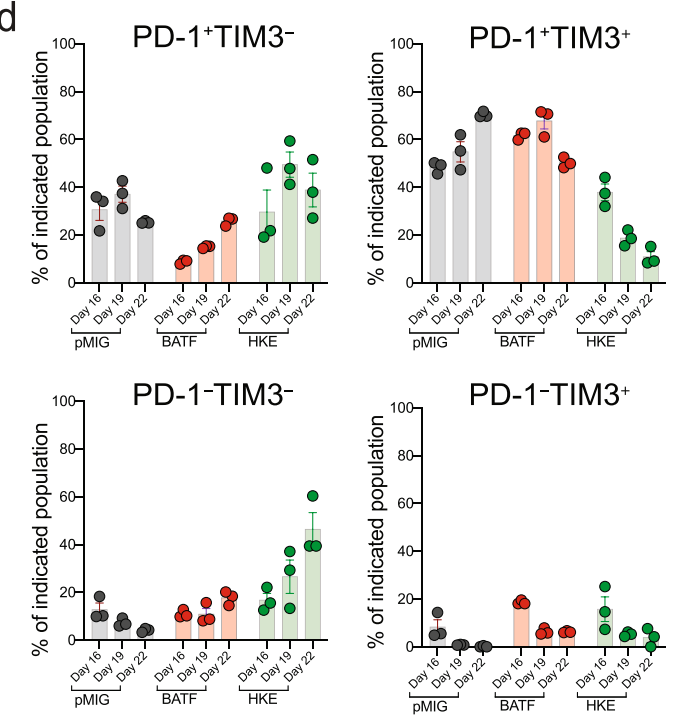

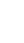


Extended Data Fig. 5 | Tumor growth and TIL expansion/survival in mice receiving BATF-transduced, BATF-HKE-transduced, or BATF-deficient CD8 ${ }^{+} \mathbf{T}$ cells. a, Tumor growth curves for the individual mice from Fig. 4b,c (PBS $(n=12)$, pMIG $(n=16), \operatorname{BATF}(n=25)$ and HKE $(n=12))$. b-f, 1×105 B16F0-hCD19 tumor cells were subcutaneously injected into the left flank of C57BL/6 mice at day 0 (D0). $100 \mu$ l of PBS, without cells or containing $1.5 \times 10^{6} \mathrm{CAR} T$ cells transduced with retroviral expression plasmids encoding either pMIG control, BATF, or BATF HKE-mutant, were adoptively transferred into C57BL/6 recipient mice by retro-orbital injection on day 12. TILs were isolated on days 13, 16, 19, and 22. c, Expression of CAR T cell marker Thy1.1 on CD8 ${ }^{+}$TILs on the indicated days. $\mathbf{d}-\mathbf{f}$, Frequencies and MFIs of the indicated PD-1- and TIM3-expressing populations from Fig. 4j,k. g-m, 1×105 B16F0-hCD19 tumor cells were injected subcutaneously into the left flank of C57BL/ 6 mice at day $0(D 0) .1 .5 \times 10^{6}$ wild-type $(W T, n=4)$ or $B A T F-$ deficient $(B A T F ~ K O, n=4) C A R$ $T$ cells were adoptively transferred at day 12. Tumor-infiltrating lymphocytes were isolated at day 20 . $\mathbf{h}$, Tumor growth curves for individual mice (dashed lines) and average (bold lines) of all tumor growth curves in a group. i-k, Contour plots of Thy1.1 expression in CD8+ TILs (i), percentages of Thy1.1+ CAR TILs (j) and numbers of Thy 1.1+ CAR TILs normalized to tumor size (k) in tumor-bearing BATF WT or BATF KO mice. I, Contour plots of PD-1 and TIM3 expression (left) and percentage of PD-1hiTIM3+ cells (right) in WT or BATF KO CAR TILs. $\mathbf{m}$, Contour plots of PD-1 and TOX expression (top left), TIM3 and TCF1 expression (bottom left), and percentages of the indicated populations (right) in WT or BATF KO CAR TILs. Data in a were obtained from three independent experiments. Each circle in $\mathbf{d}-\mathbf{f}$ and $\mathbf{j}$-m represents one mouse, and the bar graphs represent the mean \pm standard error of mean (s.e.m.). Data in $\mathbf{d}-\mathbf{m}$ are representative of two independent experiments. Data in $\mathbf{j}-\mathbf{m}$ were analyzed by two-tailed unpaired Student's $t$-test. 


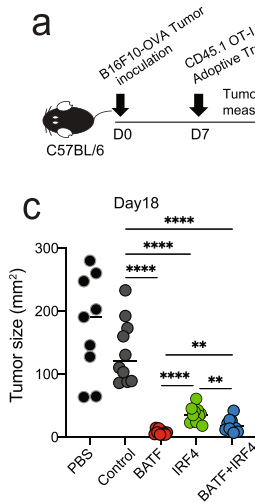

e
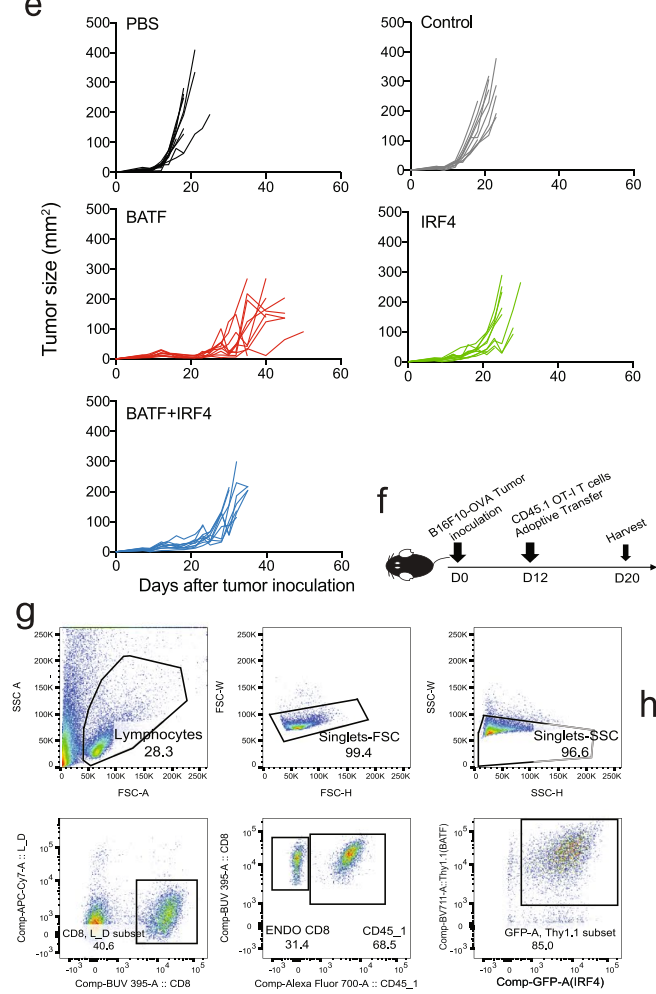

i.

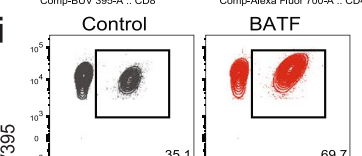

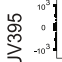

IRF4 $\frac{135.1}{\text { BATF+IRF4 }}$

Oั้

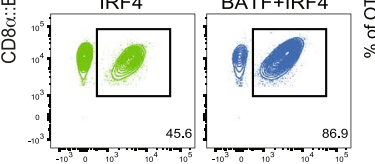

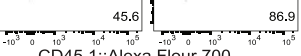

j
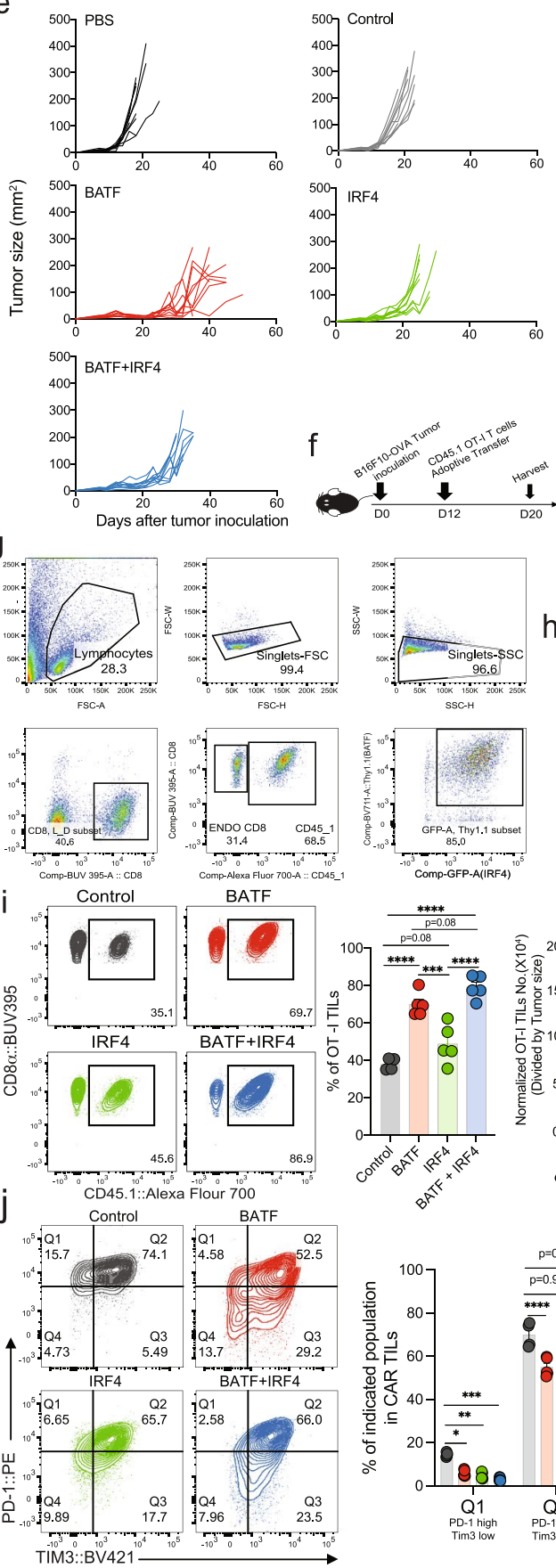
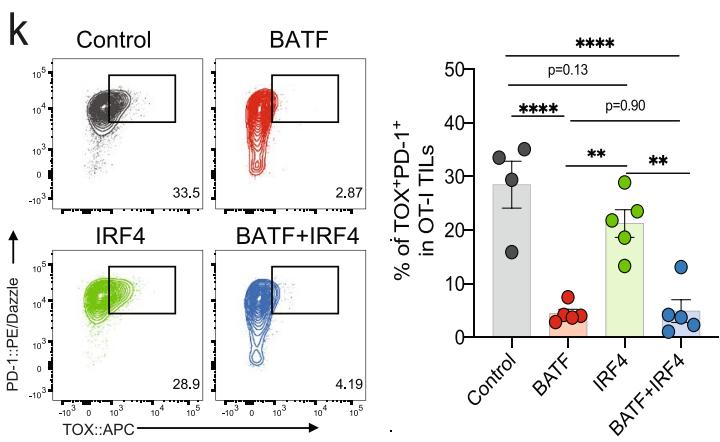

60
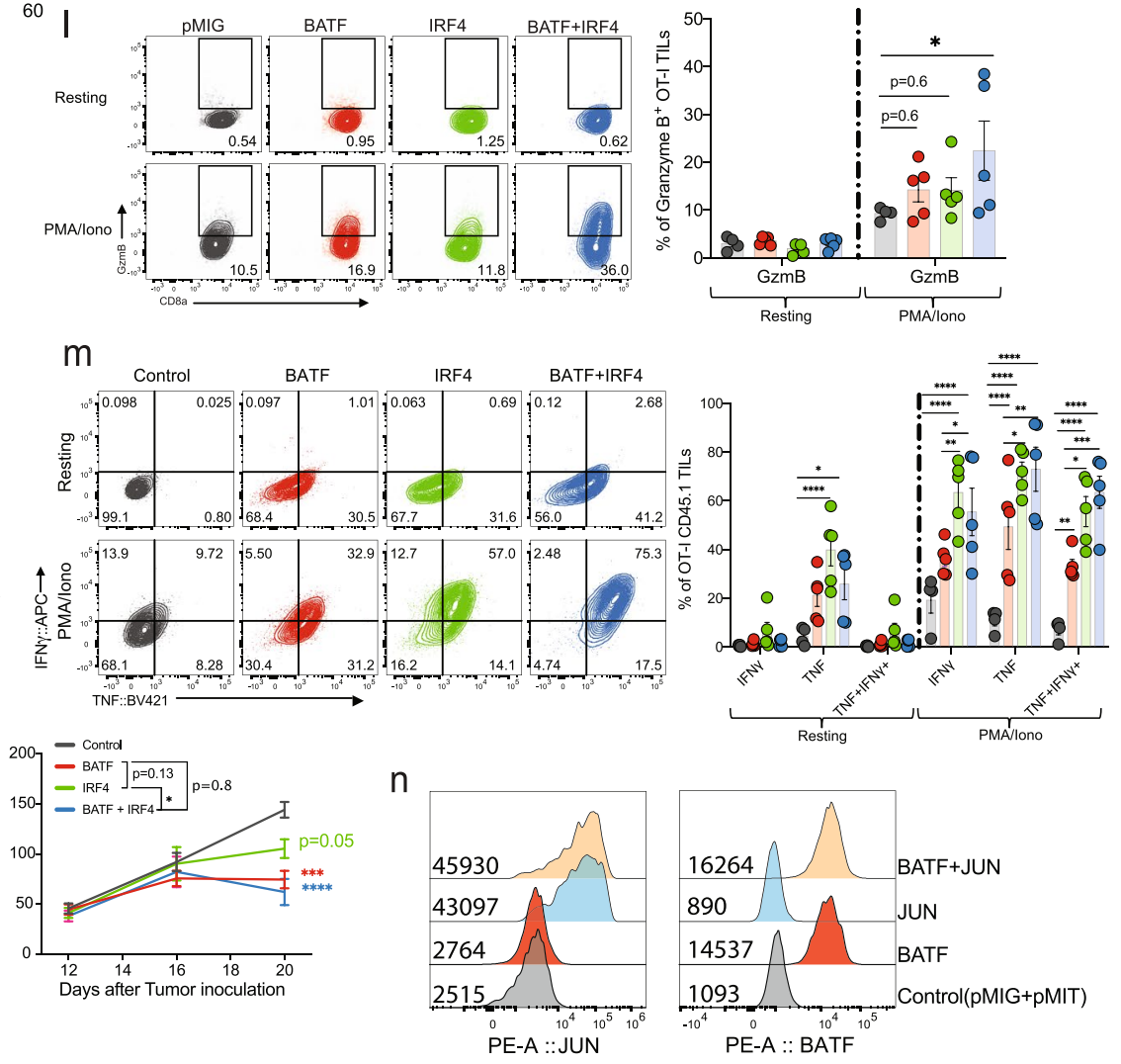

0
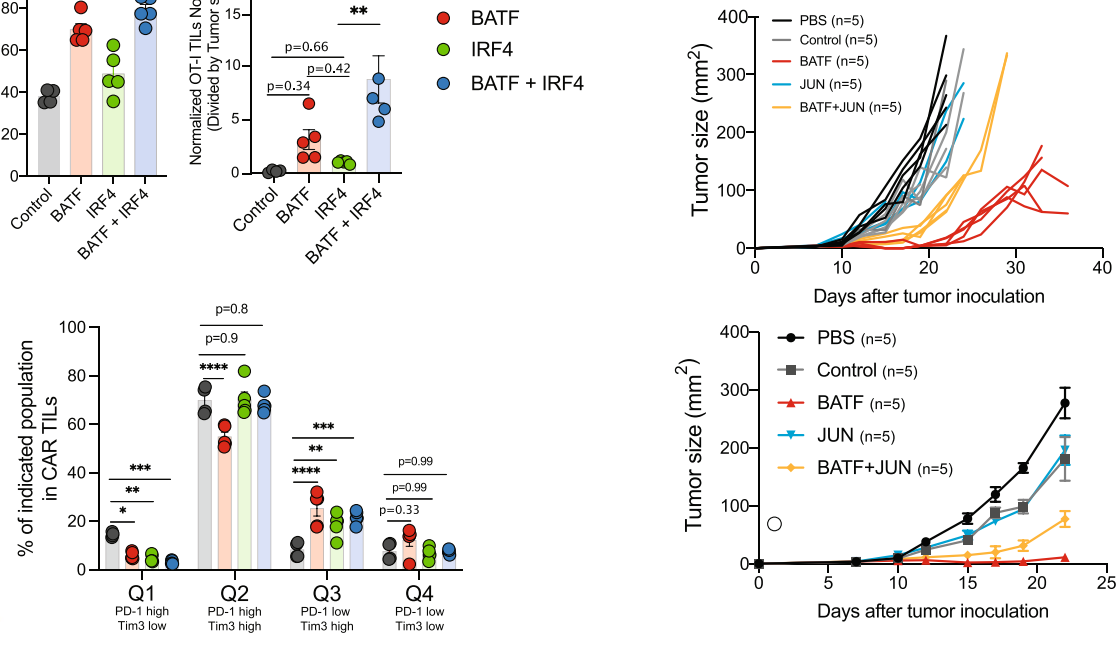

- Control

- IRF4

- BATF + IRF4

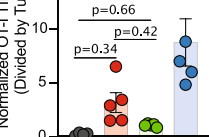

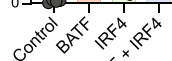

$\beta^{x^{4}}$

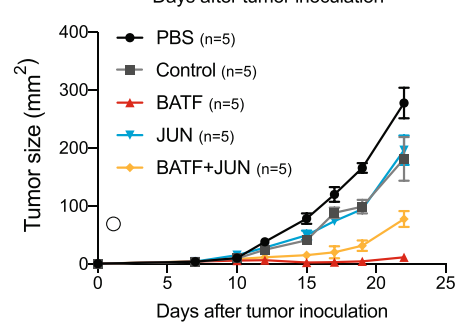

Extended Data Fig. 6 | See next page for caption. 
Extended Data Fig. 6 | Tumor growth rates, survival curves, and phenotypic analysis of CAR TILs. a-e, $2.5 \times 10^{5}$ B16F10-OVA tumor cells were injected subcutaneously into the left flank of C57BL/6 mice at day 0 (D0). $100 \mu$ of PBS $(n=10)$, without cells or containing $1 \times 10^{6}$ OT-I T cells transduced with retroviral expression plasmids encoding pMIG control $(n=10)$, BATF $(n=10)$, IRF4 $(n=10)$, or BATF + IRF4 $(n=10)$, were adoptively transferred by retroorbital injection at day 7. b. Averaged tumor growth curves for all mice in the indicated groups. c, Tumor sizes measured in individual mice at day 18. d, Mouse survival curves. e, Tumor growth curves in individual mice. $\mathbf{f}-\mathbf{m}, 2.5 \times 10^{5}$ B16F10-OVA tumor cells were injected subcutaneously into the left flank of C57BL/6 mice at day $0.1 \times 10^{6}$ pMIG control- $(n=4)$, BATF- $(n=5)$, IRF4- $(n=5)$, or BATF + IRF4-transduced $(n=5)$ OT-I cells were adoptively transferred at day 12. TILs were isolated at day 20. g, Gating strategy for flow cytometric analysis of OT-I TILs. h, Averaged tumor growth curves for all mice in the indicated groups. i, Left, Contour plots of CD8 $\alpha$ and CD45.1 expression in OT-I TILs. Middle, Percentage of OT-I TILs in CD8+ TILs. Right, Number of OT-I TILs normalized to tumor size. $\mathbf{j}$, Left, Contour plots of PD-1 and TIM3 expression in each group of OT-I TILs. Right, Percentages of the indicated PD-1- and TIM3-expressing cell populations. $\mathbf{k}$, Left, Contour plots of PD-1 and TOX expression in the indicated OT-I TILs. Right, Percentage of PD-1+TOX+ cells in OT-I TILs. I, $\mathbf{m}$, Left, Contour plots of expression of granzyme B (I) and the indicated cytokines $(\mathbf{m})$ under resting conditions or after PMA/ionomycin stimulation for $4 \mathrm{~h}$. Right, Percentages of OT-I TILs expressing granzyme B (I) or the indicated cytokines ( $\mathbf{m}$ ) under resting conditions or after PMA/ ionomycin stimulation for $4 \mathrm{~h}$. Data obtained from two biological experiments. $\mathbf{n}$, Histograms showing JUN and BATF expression in the indicated groups of transduced OT-I T cells. o, Tumor growth curves for individual mice given pMIG control-, BATF-, JUN-, or BATF + JUN-transduced OT-1 cells (top) and averaged tumor growth curves for all mice in each group (bottom). Experimental scheme as in $\mathbf{a}$. Each circle in $\mathbf{i}, \mathbf{j}, \mathbf{k}, \mathbf{I}$, and $\mathbf{m}$ represents one mouse, and the bar graphs represent the mean \pm standard error of mean (s.e.m.). Data were obtained from two independent biological experiments. Data in $\mathbf{h}, \mathbf{j}, \mathbf{I}$, and $\mathbf{m}$ were analyzed by two-way ANOVA test; data from $\mathbf{i}$ and $\mathbf{k}$, by one-way ANOVA test. ${ }^{\star} P \leq 0.05 ;{ }^{\star \star} P \leq 0.01 ;{ }^{* \star \star} P \leq 0.001 ;{ }^{\star \star \star \star} P \leq 0.0001$. 
a Higher in BATF

Exhaustion-related

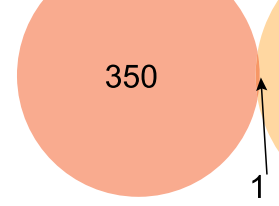

449

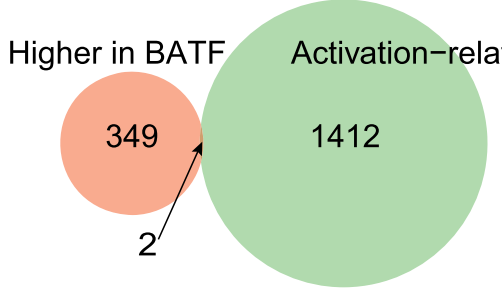

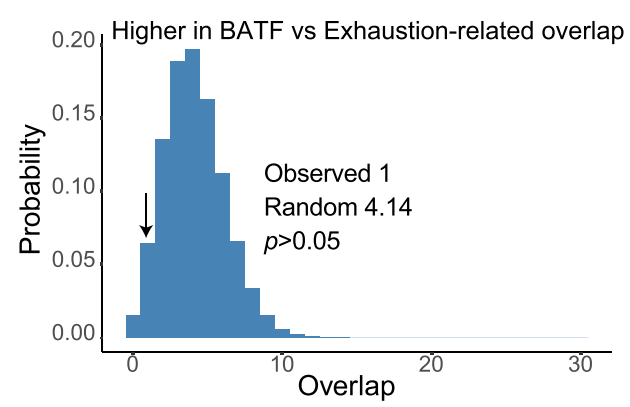

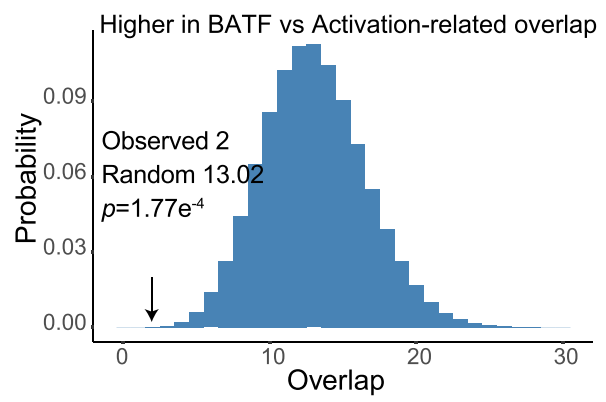

b

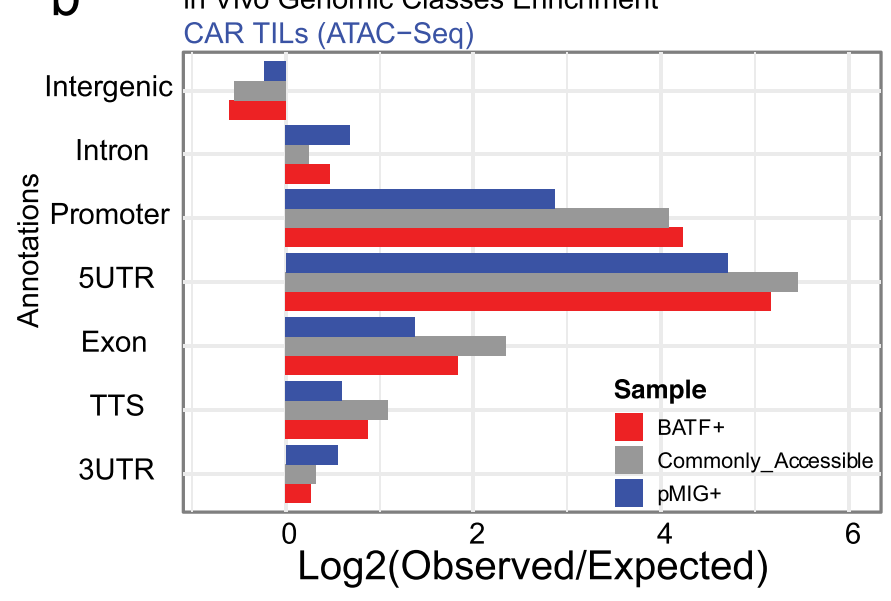

d

$$
\text { BATF>pMIG In vitro (640 DARs) }
$$

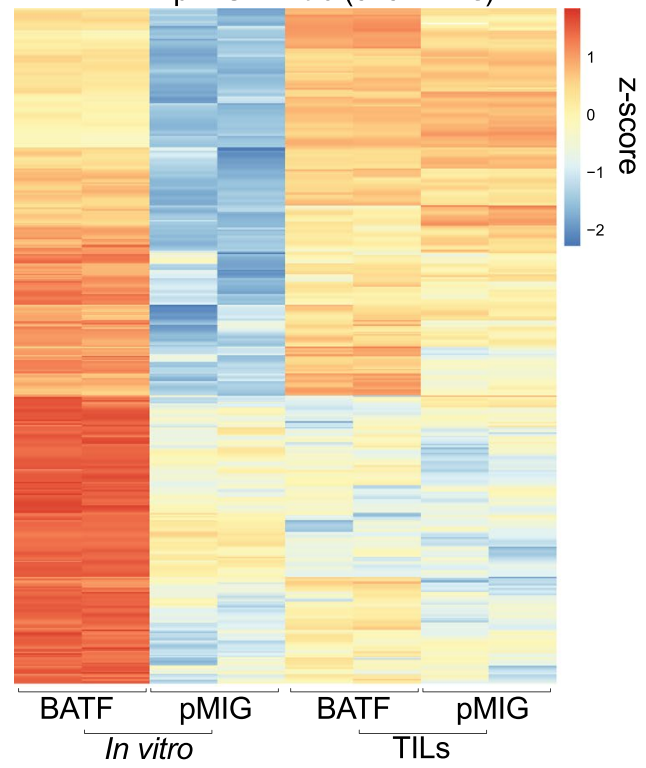

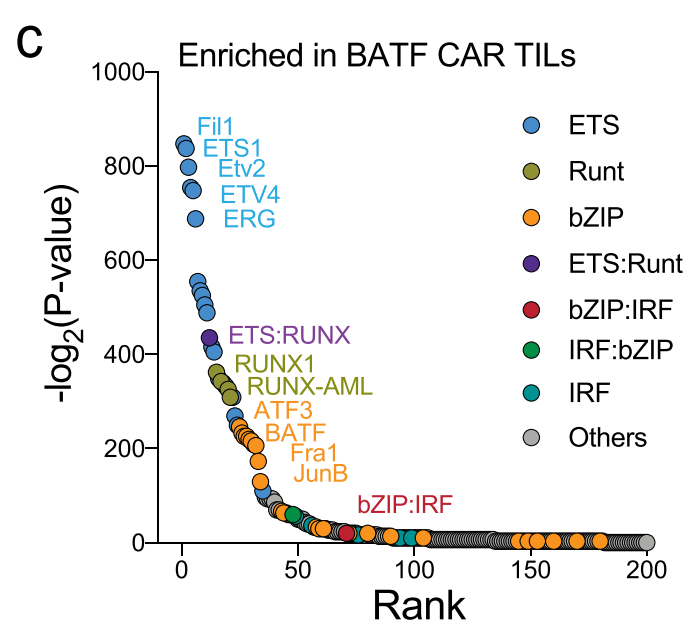

BATF>pMIG in TILs (351DARs)

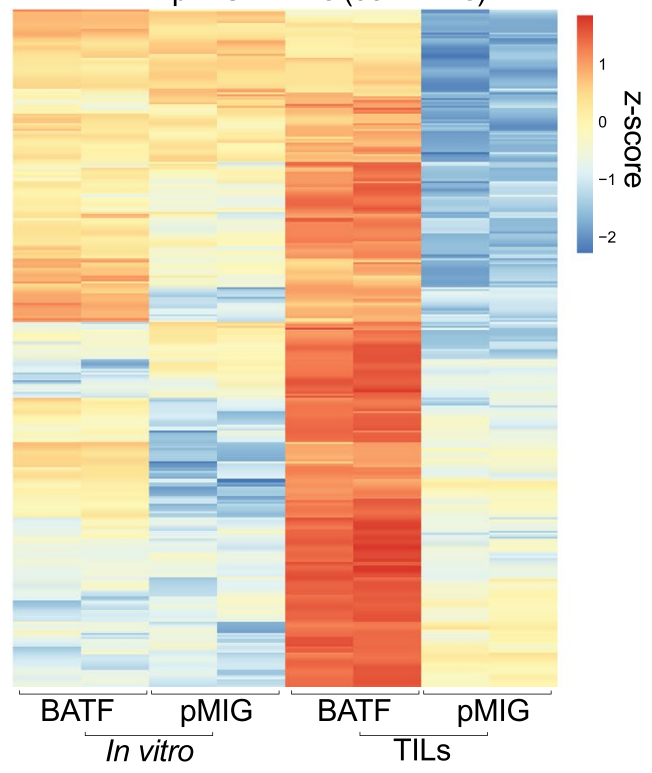

Extended Data Fig. 7 | See next page for caption. 
Extended Data Fig. 7 | Differentially accessible regions in BATF- compared to pMIG-transduced CAR TILs. a, Left, Venn diagrams showing the overlap of the 351 regions more accessible in BATF- versus pMIG-transduced TILs (Fig. 5c) with the exhaustion-related (top) or activation-related (bottom) regions from Mognol et $a^{\beta 6}$. Right, Histograms illustrate the significance calculation by one-tailed Fisher's exact test. $\mathbf{b}$, Genomic annotation of the commonly and differentially accessible regions in CAR TILs. c, Enrichment for transcription factor binding motifs in regions differentially accessible in BATF CAR TILs. d, Heatmap of ATAC-seq signal (z-score) from BATF- and pMIG-transduced CD8 ${ }^{+}$T cells or CAR TILs, in the 640 regions more accessible in BATF-transduced compared to pMIG-transduced CD8 ${ }^{+}$T cells (left; see Fig. 5b) and in the 351 regions more accessible in BATF-transduced compared to pMIG CAR TILs (right; see Fig. 5c). Each column represents a biological replicate. Data obtained from two biological experiments. 
BATF and pMIG transduced cells

BATF ChIP-seq peaks

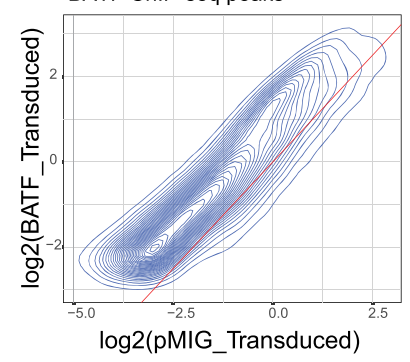

C

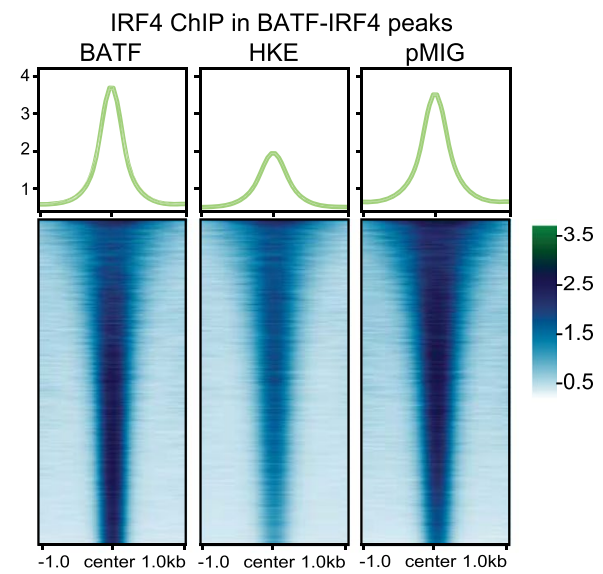

d

BATF ChIP in pMIG-IRF4 peaks

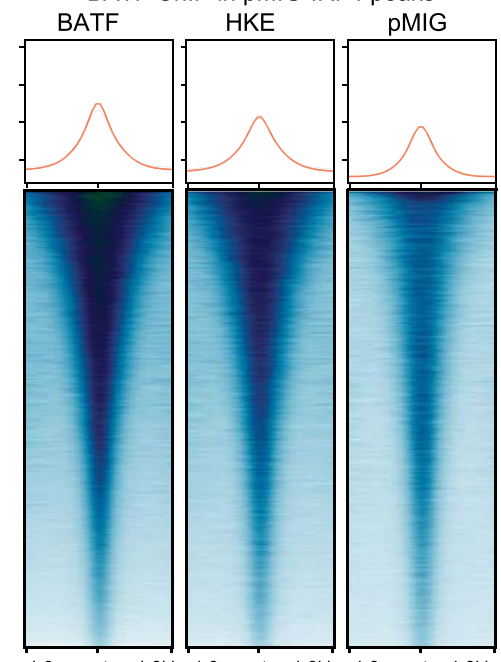

e

$10 \mathrm{~kb}$

Ctla4 1

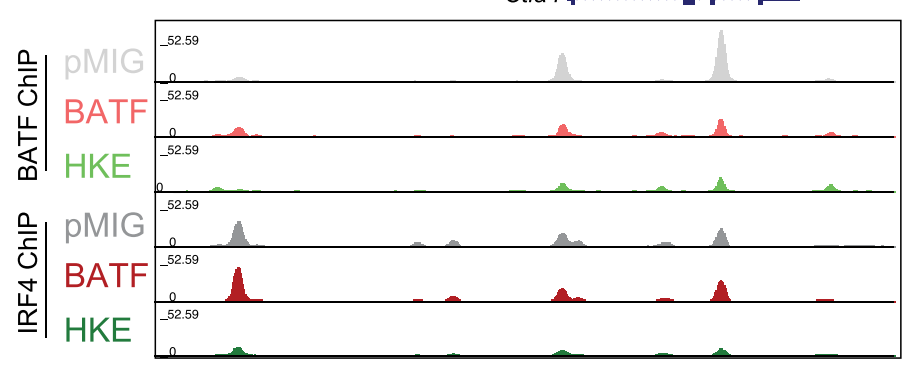

BATF-transduced cells pMIG-transduced cells Reads in BATF peaks Reads in BATF peaks $(26,451,077)$
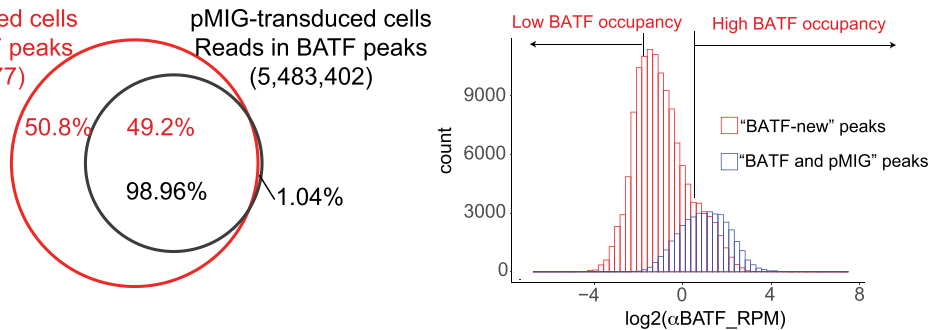

f

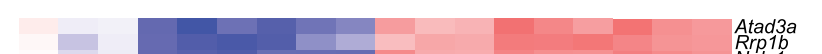
Nolc1
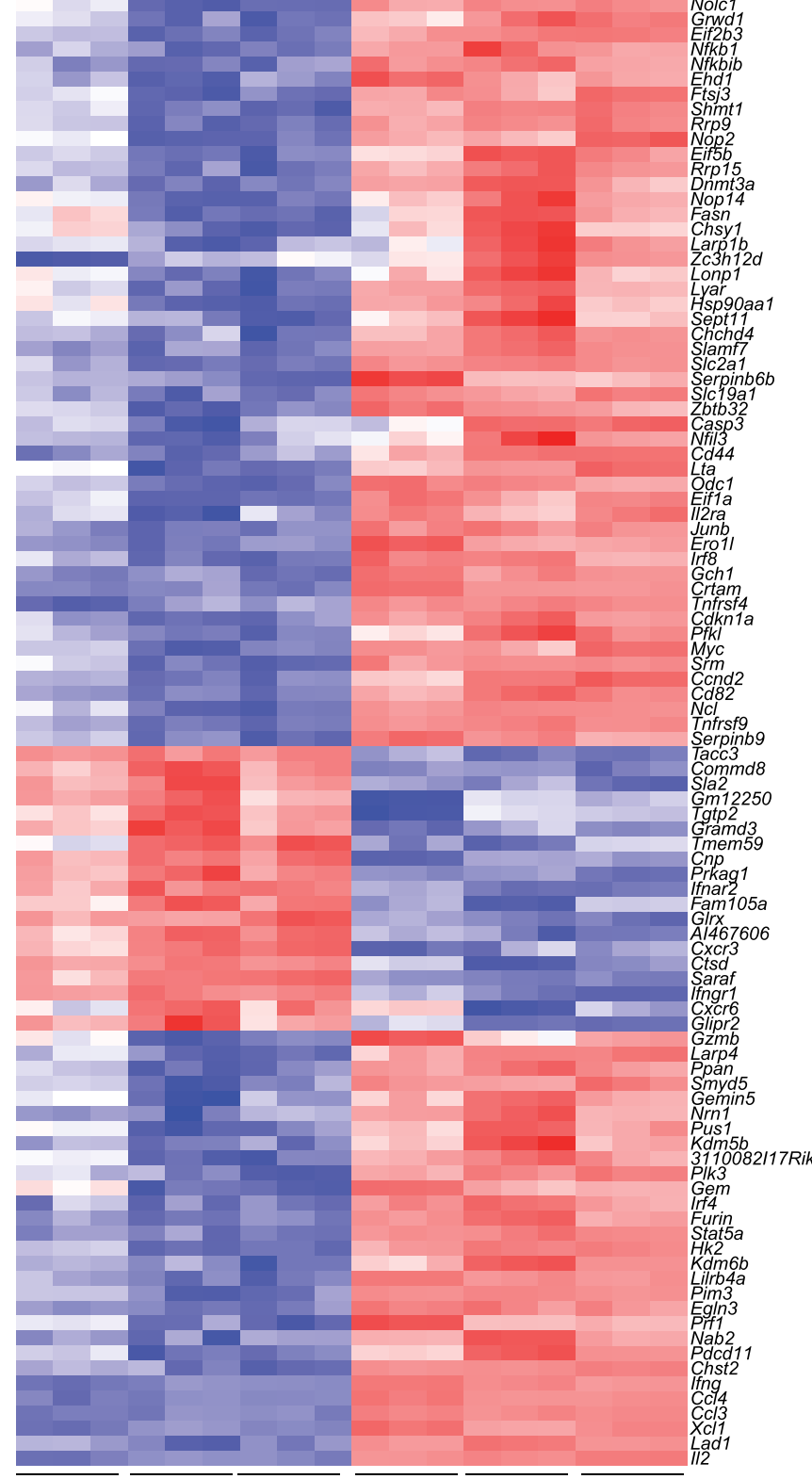

HKE Oh pMIG Oh BATF oh HKE 6h pMIG 6h BATF 6h Genes regulated by $\alpha \mathrm{CD} 3 / \alpha \mathrm{CD} 28$ stimulation in PMIG cells

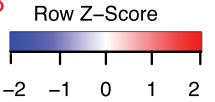

\section{Extended Data Fig. 8 | See next page for caption.}




\section{NATURE IMMUNOLOGY}

Extended Data Fig. 8 | BATF and IRF4 binding and gene expression changes in pMIG-, HKE-, and BATF-transduced cells. a, Contour plot relating BATF ChIP-seq signals $\left(\log _{2}(C P M)\right)$ in BATF-transduced CD8 ${ }^{+}$T cells to signals from the corresponding peaks in pMIG-transduced cells. $\mathbf{b}$, Left, Distribution of BATF ChIP-seq reads in peaks in BATF-transduced cells (red) versus pMIG-transduced cells (black). Right, BATF ChIP-seq signal distribution in the 'BATF and pMIG' peaks common to the two conditions (blue) and in 'BATF-new' peaks observed only in BATF-transduced cells (red). The BATF-new peaks defined here are largely the same as the BATF-only peaks in Fig. 6a. c, Heatmaps of the IRF4 ChIP-seq signal in BATF-transduced, BATF-HKE-transduced, and pMIG-transduced cells, at IRF4 peak locations called in BATF-transduced cells. Curves at the top show the average signal taken over all peaks in the respective heatmap. d, Heatmaps of the BATF ChIP-seq signal in BATF-transduced, BATF-HKE-transduced and pMIG-transduced cells, at IRF4 peak locations called in PMIG-transduced cells. Curves at the top show the average signal taken over all peaks in the respective heatmap. $\mathbf{e}$, Genome browser view of Ctla4 gene locus showing BATF and IRF4 ChIP-seq signals from pMIG-, BATF-, and HKE-transduced CD8+ T cells. f, Heatmap of normalized RNA-seq reads (as $z$-scores) under the indicated conditions, for the top 100 genes differentially expressed after $\alpha C D 3 / \alpha C D 28$ stimulation of pMIG-transduced cells. Data obtained from two or three biological experiments. 
a

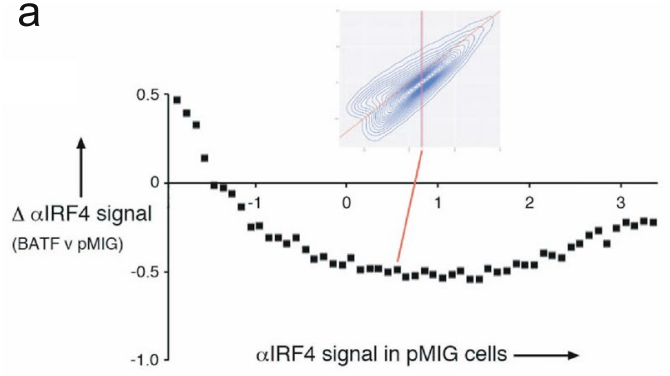

b

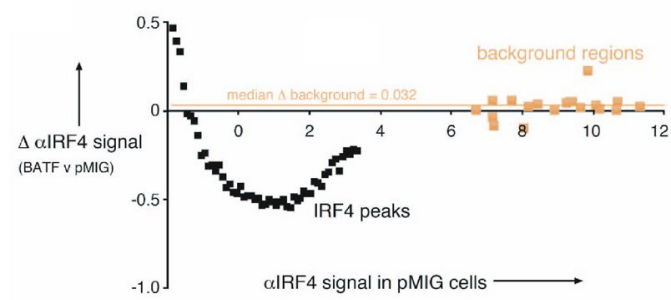

C

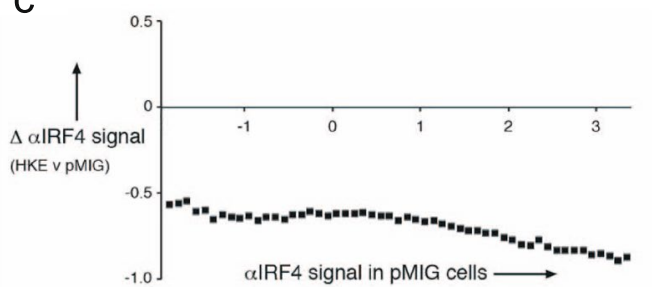

d

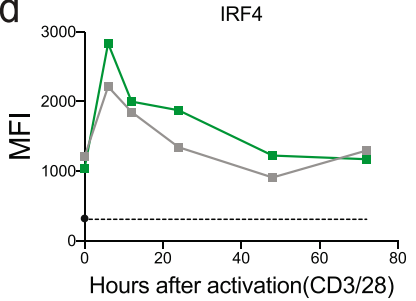

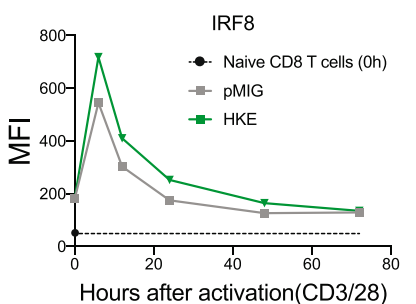

e

Definition of IRF4 ChIP Up(RED) and Down (BLUE)

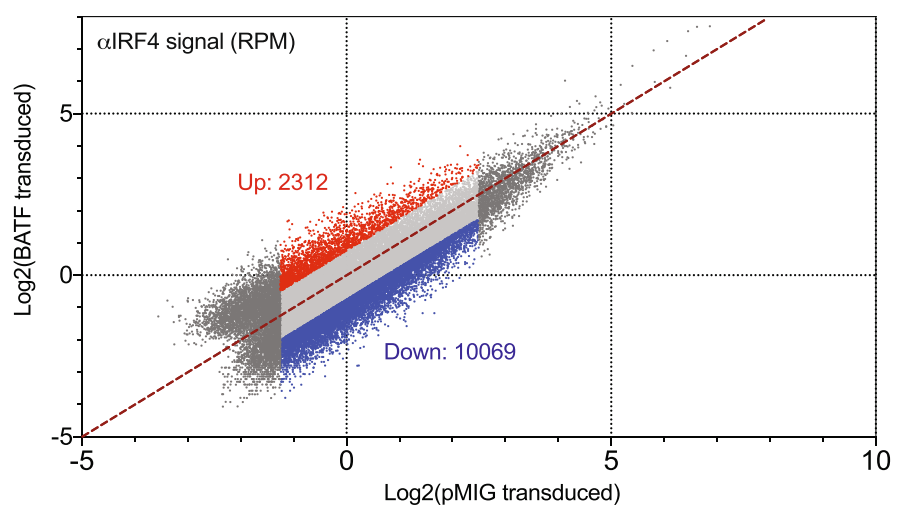

f
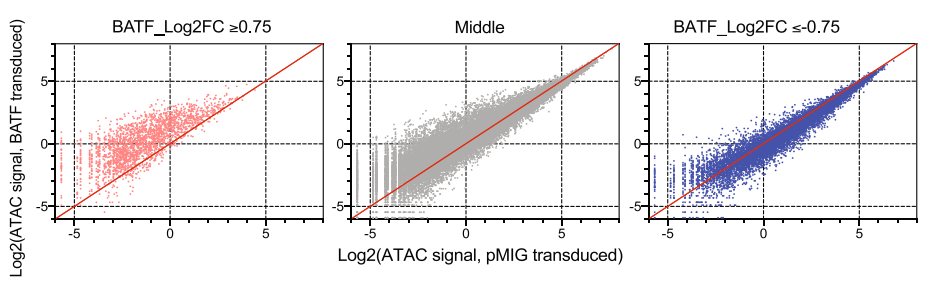

g

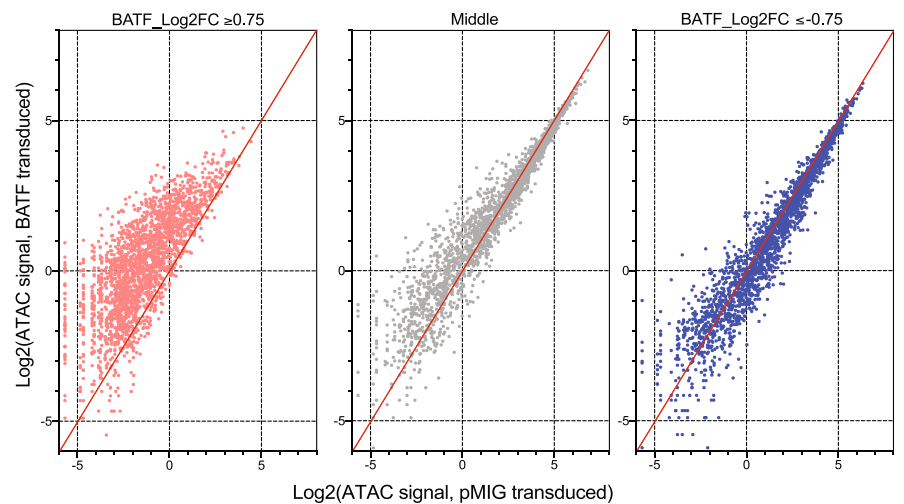

Extended Data Fig. 9 | Redistribution of IRF4 binding in BATF-overexpressing cells. a, The redistribution of the normalized IRF4 ChIP-seq signal in BATF-overexpressing cells is most evident when the median deviation of the $y$-coordinate ( $\alpha$ IRF4 ChIP-seq signal in BATF-overexpressing cells) from the diagonal in Fig. 7c, left, is plotted as a function of position on the x-axis ( $\alpha$ IRF4 ChIP-seq signal in control cells). The median for all peaks in each slice of $0.1 \log _{2}(C P M)$ units on the $x$-axis was determined. The inset replicates Fig. $7 c$, left, with a red rectangle indicating the slice between log $(C P M)=0.5$ and $\log _{2}(C P M)=0.6$ on the $x$-axis. b, Spurious ' $\alpha$ IRF4' ChIP-seq regions (as defined in Methods), incorporated into the graph of panel a. c, IRF4 does not redistribute in BATF-HKE-overexpressing cells. The median deviation of the $y$-coordinate ( $\alpha$ IRF4 ChIP-seq signal in HKE-overexpressing cells) from the diagonal in Fig. 7c, right, is plotted as a function of position on the x-axis ( $\alpha$ IRF4 ChIP-seq signal in control cells), as in a. d, IRF4 (left) and IRF8 (right) expression detected by flow cytometry (MFI) in PMIG- and HKE-transduced CD8 ${ }^{+} \mathrm{T}$ cells that had been expanded in vitro, at the indicated times after restimulation with $\alpha \mathrm{CD} 3 / \alpha \mathrm{CD} 28$. The black symbol on the $y$-axis shows expression in naïve CD8 ${ }^{+} \mathrm{T}$ cells. Values for pMIG-transduced and naïve $\mathrm{CD} 8^{+}$ T cells were obtained in the same experiments and are replotted from Fig. 6f. Overexpression of BATF-HKE did not attenuate IRF4 or IRF8 induction. e, Similar to Fig. 8a. Dot plot highlighting regions of the IRF4 ChIP-seq data from Fig. 7c where IRF4 binding increases $\left(\log _{2} \mathrm{FC} \geq 0.75\right.$, red dots), does not change substantially (light grey dots), or decreases ( $\log _{2} \mathrm{FC} \leq-0.75$, blue dots) in BATF-overexpressing relative to pMIG-transduced cells. Peaks with very low $\left(\log _{2}(\right.$ signal $\left.)<-1.25\right)$ or high $\left(\log _{2}(\right.$ signal $\left.)>2.5\right)$ IRF4 binding in pMIG cells were judged unlikely to be informative and were omitted from the analysis. $\mathbf{f}$, ATAC-seq signal (CPM) in peaks in each of the three categories defined in $\mathbf{e}$. $\mathbf{g}$, Similar to $\mathbf{f}$, but downsampling the subsets to match the 2312 regions with increased IRF4 binding in BATF-transfected cells, for clearer visualization. Data were obtained from (a-c, $\mathbf{e}-\mathbf{g}$ ), or are representative of (d), two independent biological experiments. 


\section{Reporting Summary}

Nature Research wishes to improve the reproducibility of the work that we publish. This form provides structure for consistency and transparency in reporting. For further information on Nature Research policies, see our Editorial Policies and the Editorial Policy Checklist.

\section{Statistics}

For all statistical analyses, confirm that the following items are present in the figure legend, table legend, main text, or Methods section.

$\mathrm{n} / \mathrm{a}$ Confirmed

$\bigotimes$ The exact sample size $(n)$ for each experimental group/condition, given as a discrete number and unit of measurement

\ A statement on whether measurements were taken from distinct samples or whether the same sample was measured repeatedly

The statistical test(s) used AND whether they are one- or two-sided

Only common tests should be described solely by name; describe more complex techniques in the Methods section.

Х A description of all covariates tested

\A description of any assumptions or corrections, such as tests of normality and adjustment for multiple comparisons

$\triangle$ A full description of the statistical parameters including central tendency (e.g. means) or other basic estimates (e.g. regression coefficient)

AND variation (e.g. standard deviation) or associated estimates of uncertainty (e.g. confidence intervals)

$X$ For null hypothesis testing, the test statistic (e.g. $F, t, r$ ) with confidence intervals, effect sizes, degrees of freedom and $P$ value noted Give $P$ values as exact values whenever suitable.

Х $\square$ For Bayesian analysis, information on the choice of priors and Markov chain Monte Carlo settings

$\square$ For hierarchical and complex designs, identification of the appropriate level for tests and full reporting of outcomes

X| $\square$ Estimates of effect sizes (e.g. Cohen's $d$, Pearson's $r$ ), indicating how they were calculated

\section{Our web collection on statistics for biologists contains articles on many of the points above.}

\section{Software and code}

Policy information about availability of computer code

Data collection BD LSR Fortessa, BD LSR-II, BD facscelesta, BD FACS ARIA II and III, Illumina Novaseq 6000,

Data analysis ATAC-seq and ChiP seq analysis: Bowtie(version 1.0.0 and -X 2000 -m 1 --best --strata -tryhard -S -fr), samtools(v1.8), TrimGalore, Picard(v1.94), bedtools(2.27.1), MACS2(version 2.1.1.20160309), Java Genomics Toolkit, R (version 3.5.2)and BioConductor packages ( 1.3 .1 ) (GenomicRanges, GenomicAlignments, Rsamtools, limma, pheatmap, ggplot2, gtools, RColorBrewer, gtools, reshape2, RColorBrewer, MEDIPS)

RNA-seq : TrimGalore v0.4.5, Cutadapt v1.13, STAR v2.5.3a, R v3.5.2, GSEA v3.0; BioConductor packages: (for data analysis), rtracklayer v1.34.2, GenomicAlignments v1.10.1, DESeq2 v1.14.1, IRdisplay v0.7.0, limma v3.38.3, edgeR v3.24.3, Mus.musculus v1.3.1, GenomicRanges v1.34.0, Glimma v1.10.1, stringr v1.4.0; BioConductor packages: (for making figures) pheatmap v1.0.12, ggplot2 v3.3.2, ggrepel v0.9.0, viridis v0.5.1, viridisLite v0.3.0, grid, RColorBrewer v1.1-2.

Flow cytometry analysis: FlowJo v.10 (Tree Star, Inc), Prism 8 (GraphPad Software)

Tumor growth curve / survival curve analysis: Prism 8 (GraphPad Software) 
Policy information about availability of data

All manuscripts must include a data availability statement. This statement should provide the following information, where applicable:

- Accession codes, unique identifiers, or web links for publicly available datasets

- A list of figures that have associated raw data

- A description of any restrictions on data availability

ChiP-seq, ATAC-seq and RNA-seq is available after publication (GSE154747). The other data are provided in supplementary source data figures. Additional information and materials will be made available upon request.

\section{Field-specific reporting}

Please select the one below that is the best fit for your research. If you are not sure, read the appropriate sections before making your selection.

Х Life sciences

Behavioural \& social sciences

Ecological, evolutionary \& environmental sciences

For a reference copy of the document with all sections, see nature.com/documents/nr-reporting-summary-flat.pdf

\section{Life sciences study design}

All studies must disclose on these points even when the disclosure is negative.

Sample size Sample sizes were not predetermined; for the OT-I and CAR mouse experiments, sample sizes were chosen based on a previous study from our lab. Basically, We used at least 3 biological samples to achieve $95 \%$ power for detecting statistical differences.

Data exclusions No data point excluded

Replication $\quad$ All experimental findings can be and were reliably reproduced. For sequencing, CyTOF and flow cytometry, we performed at least two independent biological replicates of each assay and all results were reproducible.

Randomization Both female and male mice (7weeks-12weeks of age) were used with sex- and age-matched. And mice were randomly allocated to each experiments.

Blinding In most cases, tumor sizes were measured in a blinded manner by DLAC staff except during the holiday season or when the institute was under restricted access due to the COVID-19 shut-down. Investigators were not blinded to group allocation during data collection or analysis to avoid any mislabeling of different groups

\section{Reporting for specific materials, systems and methods}

We require information from authors about some types of materials, experimental systems and methods used in many studies. Here, indicate whether each material, system or method listed is relevant to your study. If you are not sure if a list item applies to your research, read the appropriate section before selecting a response.

\begin{tabular}{|c|c|c|c|}
\hline \multicolumn{2}{|r|}{ Materials \& experimental systems } & \multicolumn{2}{|c|}{ Methods } \\
\hline $\mathrm{n} / \mathrm{a}$ & Involved in the study & $\mathrm{n} / \mathrm{a}$ & Involved in the study \\
\hline$\square$ & $\bigotimes$ Antibodies & ${ }^{-}$ & $\bigotimes$ ChIP-seq \\
\hline \begin{tabular}{|c|cc} 
\\
\end{tabular} & $\bigotimes$ Eukaryotic cell lines & L & \ Flow cytometry \\
\hline Х & Palaeontology and archaeology & Х & $\square$ MRI-based neuroimaging \\
\hline
\end{tabular}

$\square \bigotimes$ Animals and other organisms

$\square$ \uman research participants

\ $\square$ Clinical data

$\bigotimes \square$ Dual use research of concern

\section{Antibodies}

Antibodies used

Flow cytometry antibodies
Fluorescence Target Clone Dilution Product ID Company
BUV 395 CD8a 53-6.7 1:200 563786 BD Bioscience
BV 711 Thy1.1 OX-7 1:200 202539 Biolegend
BV 711 CD45.1 A20 1:200 110739 Biolegend
BV 421 TIM3 RMT3-23 1:200 119723 Biolegend
BV 421 CD62L MEL-14 1:200 104436 Biolegend


BV 421 T-bet O4-46 1:200 563318 BD Bioscience BV 421 TNF MP5-XT22 1:200 506328 Biolegend

eFlour 450 Nur77 12.14 1:200 46-5965-82 eBioscience

eFlour 450 IRF4 $3 E 4$ 1:200 48-9858-82 eBioscience

Percp-eFlour 710 EOMES Dan11mag 1:100 46-4875-82 eBioscience

Percp-Cy5.5 2B4 m2B4(B6)458.1 1:200 133514 Biolegend

Percp-Cy5.5 CD44 IM7 1:200 103032 Biolegend

Percp-Cy5.5 IFN-g XMG1.2 1:200 505822 Biolegend

PE PD-1 29F.1A12 1:200 135206 Biolegend

PE/Dazzle PD-1 29F.1A12 1:200 135228 Biolegend

PE Nur77 12.14 1:200 12-5965-82 eBioscience

PE IL7r A7R34 1:200 135010 Biolegend

PE TOX TXRX10 1:200 12-6502-82 eBioscience

PE C-JUN 60A8 1:200 15683 BD Bioscience

PE TCF1/TCF7 C63D9 1:200 14456 Cell Signalling Technology

PE BATF S39-1060 1:200 564503 BD Bioscience

PE NOR1 H-7 1:200 sc-393902 Santacruz Biotech

PE Granzyme B QA16A02 1:20 372208 Biolegend

PE/Cy7 CD107a 1D4B 1:200 121620 Biolegend

PE/CY7 TIGIT 1 G9 1:200 142108 Biolegend

PE/Cy7 KI67 16A8 1:200 652426 Biolegend

PE/Cy7 PD-1 29F.1A12 1:200 621616 Biolegend

APC KLRG1 2F1/KLRG1 1:200 138412 Biolegend

APC TCF1/TCF7 C63D9 1:200 37636 Biolegend

AF647 TOX REA473 1:200 130-118-335 Miltenyi Biotech

AF647 Nurr1 F-5 1:200 sc-376984 Santacruz Biotech

Purified(Primary) MAFF N/A 1:200 12771-1-AP Thermo

AF647 Rabbit-IgG N/A 1:200 A-21244 Thermo

APC LAG3 C9B7W 1:200 125210 Biolegend

APC IFN-g XMG1.2 1:200 505810 Biolegend

AF700 CD45.1 A20 1:200 110724 Biolegend

CyTOF antibodies

Metal Target Clone Dilution Product ID Company

089 Y CD45 30-F11 1:200 3089005B Fluidigm

115 In CD3e 145-2C11 1:100 100345 Biolegend

142 Nd CXCR5 L138D7 1:100 3142015B Fluidigm

143Nd CD90.1 OX7 1:200 202501 Biolegend

146Nd CD4 RM4-5 1:200 100561 Biolegend

147 Sm KLRG1 2F1 1:200 16-5893-82 Thermo

$148 \mathrm{Nd}$ CD11b M1/70 1:200 101249 Biolegend

150 Nd CD27 LG.3A.10 1:200 3150017B Fluidigm

151 Eu CD25(IL2Ra) 3C7 1:100 3151007B Fluidigm

152 Sm TCF7/TCF1 C63D9 1:200 2203BF Cell Signalling Technology

153 Eu CD8a 53-6.7 1:200 3153012B Fluidigm

154 sm BATF D7C5 1:100 3154012A Fluidigm

155 Gd TOX TXRX10 1:100 14-6502-82 Thermo

156 Gd CD28 37.51 1:200 102119 Biolegend

158 Gd Foxp3 FJK16s 1:100 3158003A Fluidigm

159 Tb PD-1 29F.1A12 1:200 3159024B Fluidigm

160 Gd CD62L MEL14 1:200 3160008B Fluidigm

162 Dy TIM3 RMT323 1:200 3162029B Fluidigm

163 Dy ICOS 7E.17G9 1:200 117407 Biolegend

164 Dy NK1.1 PK136 1:100 108743 Biolegend

165 Ho CD160 7H1 1:100 143002 Biolegend

166 Er F4/80 BM8 1:200 123143 Biolegend

167 Er IL7r A7R34 1:200 135029 Biolegend

170 Er CD40L MR1 1:100 3170011B Fluidigm

171 Yb CD44 IM7 1:200 3171003B Fluidigm

173 Yb Granzyme B GB11 1:100 3173006B Fluidigm

174 Yb LAG3 C9B7W 1:100 3174019B Fluidigm

175 Lu CD38 90 1:100 3175014B Fluidigm

176 Yb cMYC $9 E 10$ 1:100 3176012B Fluidigm

Validation

All antibodies are commercially available, and have been validated by manufacturer

Eukaryotic cell lines

Policy information about cell lines

Cell line source(s)

The B16F0 mouse melanoma cell line were purchased from ATCC(ATCC ${ }^{\circledR}$ CRL6322 $^{\mathrm{TM}}$ ). 
Authentication

Mycoplasma contamination

Commonly misidentified lines (See $\underline{I C L A C}$ register)
Plat-E cells were not authentication.B16FO cell line obtained from ATCC were authenticated by the manufacturer. and B16 line used in the study were verified by microscopy(morphology) and flow cytometry (hCD19 expression). B16F0-hCD19 cell line stained positive for hCD19, and B16F10-OVA cell line stained negative for hCD19.

\section{Every cell lines tested negative for mycoplasma contamination}

No commonly misidentified lines were used in this study

\section{Animals and other organisms}

Policy information about studies involving animals; ARRIVE guidelines recommended for reporting animal research

Laboratory animals C57BL6/J, B6.SJL-PtprcaPepcb/BoyJ(CD45.1), and C57BL/6-Tg(TcraTcrb)1100Mjb/J(OT-I) were obtained from Jackson Laboratory. CD45.1XOT-I mice were crossbred with CD45.1 and OT-I mice in the institute. Male mouse used for experiments on anti-tumor effects, and both male and female mouse used for other in vitro studies. 6 week-old mice were purchased from Jackson Laboratories to be used as recipient mice, and rested for at least one week after delivery before being used for experiments. Mice were agematched and between 7-12 weeks old when used for experiments. Mice were maintained with specific pathogen-free conditions at Animal Room in La Jolla Institute for Immunology. Mice were housed at an ambient temperature of approximately $22^{\sim} 23^{\circ} \mathrm{C}$, a humidity of $40 \%$ to $60 \%$, and a light dart cycle of 12 hours.

Wild animals

No wild animal were used in the study

Field-collected samples No field collected samples were used in the study

Ethics oversight

All mice were bred and/or managed in the animal facility at the La Jolla Institute for Immunology. All experiments were performed in compliance with the study protocol signed by the La Jolla Institute Institutional Animal Care and Use Committee (IACUC) regulations.

Note that full information on the approval of the study protocol must also be provided in the manuscript.

\section{Human research participants}

\section{Policy information about studies involving human research participants}

Population characteristics

Whole blood samples from healthy subjects were collected by a staff phlebotomist in the Clinical Studies Core at the La Jolla Institute for Immunology

Recruitment

. Donors were compensated per La Jolla Institute policy.

Ethics oversight

Approval for human studies: La Jolla Institute Institutional Review Board (IRB), protocol number SCRO_002. Informed consent was obtained from all human blood donors

Note that full information on the approval of the study protocol must also be provided in the manuscript.

\section{ChIP-seq}

\section{Data deposition}

\Confirm that both raw and final processed data have been deposited in a public database such as GEO.

$\bigotimes$ Confirm that you have deposited or provided access to graph files (e.g. BED files) for the called peaks.

Data access links

May remain private before publication.

Files in database submission

Genome browser session (e.g. $\underline{\operatorname{UCSC}}$ )
To review GEO accession GSE154747:

Go to https://www.ncbi.nlm.nih.gov/geo/query/acc.cgi?acc=GSE154747

Enter token ehqxsymertihtsp into the box

BATF-aBATF_rep1, BATF-aBATF_rep2, BATF-aIRF4_rep1, BATF-aIRF4_rep2, pMIG-aBATF_rep1, pMIG-aBATF_rep2,pMIGaIRF4_rep1, pMIGF-aIRF4_rep2, BATF-ReStimulated_6h-aIRF4-rep1, BATF-ReStimulated_6h-aIRF4-rep2, HKEReStimulated_6h-aIRF4-rep1, HKE-ReStimulated_6h-aIRF4-rep2, pMIG-ReStimulated_6h-alRF4-rep1, pMIG-ReStimulated_6haIRF4-rep2, BATF-ReStimulated_6h-aBATF-1, BATF-ReStimulated_6h-aBATF-2, HKE-ReStimulated_6h-aBATF-1, HKEReStimulated_6h-aBATF-2, pMIG-ReStimulated_6h-aBATF-1, pMIG-ReStimulated_6h-aBATF-2, BATF-ReStimulated_6h-inputrep1, BATF-ReStimulated_6h-input-rep2, HKE-ReStimulated_6h-input-rep1, HKE-ReStimulated_6h-input-rep2, pMIGReStimulated_6h-input-rep1, pMIG-ReStimulated_6h-input-rep2

we will provide the link upon the reviewer's request

\section{Methodology}


Antibodies

Rabbit Anti- BATF brookwoodbiomedical

IRF-4 (D9P5H) Rabbit mAb \#15106, Cell signaling technology

Peak calling parameters

For each sample, peaks were called using MACS2 (version 2.1.1.20160309) callpeak function, using the sample's respective input dataset, qvalue of 0.05 --keep-dup all and --nomodel parameters. The narrowpeak files among replicates were merged using bedtools merge (version v2.27.1).

Data quality
Data Quality:
All used samples had a quality score of at least 1. TapeStation before library sequencing. shown in the attached file. above 1 ( $1 \%$ of RiP).
Total Peaks Sample Name
21289 BATF-aBATF_rep1
24242 BATF-aBATF_rep2
817 BATF-aIRF4_rep1
115 BATF-aIRF4_rep2
899 pMIG-aBATF_rep1
668 pMIG-aBATF_rep2
328 pMIG-aIRF4_rep1
610 pMIG-aIRF4_rep2
23775 BATF-ReStimulated_6h-alRF4-rep1
28448 BATF-ReStimulated_6h-alRF4-rep2
19563 HKE-ReStimulated_6h-aIRF4-rep1
25672 HKE-ReStimulated_6h-alRF4-rep2
32777 pMIG-ReStimulated_6h-alRF4-rep1
39380 pMIG-ReStimulated_6h-alRF4-rep2
17190 BATF-ReStimulated_6h-aBATF-rep1
17329 BATF-ReStimulated_6h-aBATF-rep2
13002 HKE-ReStimulated_6h-aBATF-rep1
11875 HKE-ReStimulated_6h-aBATF-rep2
4938 pMIG-ReStimulated_6h-aBATF-rep1
4609 pMIG-ReStimulated_6h-aBATF-rep2

All individual samples had to obtain a quality tag of higher than

0 (scale from -2 to 2) when computing enrichment and quality measures from the phantompeakqualtools.

The computationally obtained fragment size was checked to be in line to what the experimented prepared and saw in the

Peak calling was done using MACS with a minimum FDR (q-value) cutoff for peak detection of 0.05 (5\%). Total peaks per sample

By analysing the Reads in Peaks (RiP) value obtained from ChIPQC's R package, all samples were controlled. All samples had values

Software

Peaks from BATF-transduced CD8+ T cells subjected to ChIP-Seq with anti-BATF antibodies were functionally annotated to the mm10 using HOMER annotatePeak.pl program. Distance to nearest TSS and gene name were filtered from the annotation results. A sublist of the genes differentially expressed between BATF and pMIG-transduced CD8+ T cells, identified by RNA-Seq analysis, was used to subset separately the peak annotation results for genes upregulated and downregulated in BATF-transduced cells. The genomic histograms were generated using R (3.5.2) with all the peak results, whereas the upregulated and downregulated histograms used the subset of genes generated above. The percentage of genes closer than $20 \mathrm{~kb}$ was obtaining by taking the absolute value to the closest TSS that was lower than or equal to $20000 \mathrm{bp}$. The distances were numerically sorted and an empirical cumulative distribution function was generated based on the data.

\section{Flow Cytometry}

\section{Plots}

Confirm that:

\The axis labels state the marker and fluorochrome used (e.g. CD4-FITC).

\The axis scales are clearly visible. Include numbers along axes only for bottom left plot of group (a 'group' is an analysis of identical markers).

\All plots are contour plots with outliers or pseudocolor plots.

\A numerical value for number of cells or percentage (with statistics) is provided.

\section{Methodology}

Sample preparation lymph nodes and spleens were mechanically digested, filtered into single cell suspensions, and stained using antibodies. Tumor infiltrating lymphocytes were collected as below.

tumors were collected from the mice and tumors were placed into C tubes (Miltenyi Biotec) containing RPMI 1640 with 10\% FBS and Collagenase D (1 mg/mL; Roche), hyaluronidase (30 unit/mL; Sigma-Aldrich), and DNase I (100 $\mathrm{mg} / \mathrm{mL}$; SigmaAldrich). Tumors were dissociated using the gentle MACS dissociator (Milteny Biotech), incubated with shaking at $2000 \mathrm{rpm}$ 
for $60 \mathrm{~min}$ at $37^{\circ} \mathrm{C}$, filtered through a 70 ?M filter and spun down. Lymphocytes were separated using lymphocyte separation medium (MP Biomedicals, cat. no.: 0850494).

Instrument

Software

Cell population abundance

Gating strategy
LSRFortessa, LSR-II, FACSAria-I, FACSAria-II, FACSA ria-Fusion (BD Biosciences)

the flow cytometry data. FACSDiva8.0 (BD Biosciences), FlowJo v.10 (Tree Star, Inc), Prism 8 (GraphPad Software)

Cell sorting was performed by the Ul flow cytometry core, using FACS ARIA-I, FACS ARIA-II, or FACS Aria-fusion (BD Biosciences) flow cytometers. For transcriptional profiling using Smart-seq, 10,000 cells were sorted from the Live/Dead dyenegative CD8+Thy1.1+GFP+ population of the isolated tumor infiltrating lymphocytes or cultured CD8+ T cells. The cells were resuspended in FACS buffer and filtered with a 70 a M filter before sorting. For ATAC-seq, 50,000 live cells were sorted using the same procedure as for Smart-seq. Cells were sorted into $1.5 \mathrm{ml}$ microfuge tubes containing $500 \mu \mathrm{ll} 50 \%$ FBS. The sorted cells were washed with cold PBS twice before further procedures.

At least 100,000 cell were collected for flow cytometry analysis

Gating strategy for cell sorting:

CAR sorts: FSC-A/SSC-A $\rightarrow$ FSC-W/FSC-H $\rightarrow$ SSC-W/SSC-H $\rightarrow$ (Live) CD $8+$ Live/dead dye neg. $->$ GFP+ Thy1.1+ (CAR)

Gating strategy for flow cytometry: Gating strategy for all experiments are similar

TILs, surface markers, cytokines or transcription factor expression: FSC-A/SSC-A $\rightarrow$ FSC-W/ FSC-H $\rightarrow$ SSC-W/SSC-H $\rightarrow$ (Live) CD8+ Live/dead dye neg. -> GFP+ Thy1.1+ or CD45.1+GFP+

\Tick this box to confirm that a figure exemplifying the gating strategy is provided in the Supplementary Information. 